\title{
Ecological Data Collected in the Santee River Basin and Coastal Drainages, North and South Carolina, 1996-98
}

By T.A. Abrahamsen

U.S. Geological Survey

Open-File Report 01-352

Prepared as part of the

National Water-Quality Assessment Program

Santee River Basin and Coastal Drainages Study Unit 


\section{U.S. DEPARTMENT OF THE INTERIOR \\ GALE A. NORTON, Secretary}

U.S. GEOLOGICAL SURVEY

Charles G. Groat, Director

Use of trade, product, or firm names in this publication is for descriptive purposes only and does not imply endorsement by the U.S. Geological Survey

For additional information write to:

District Chief

U.S. Geological Survey

720 Gracern Road

Columbia, SC 29210-7651
Copies of this report can be purchased from:

U.S. Geological Survey Branch of Information Services

Box 25286

Denver, CO 80225

888-ASK-USGS

Additional information about water resources in South Carolina is available on the World Wide Web at http://sc.water.usgs.gov 


\section{FOREWORD}

The U.S. Geological Survey (USGS) is committed to serve the Nation with accurate and timely scientific information that helps enhance and protect the overall quality of life, and facilitates effective management of water, biological, energy, and mineral resources. Information on the quality of the Nation's water resources is of critical interest to the USGS because it is so integrally linked to the long-term availability of water that is clean and safe for drinking and recreation and that is suitable for industry, irrigation, and habitat for fishes and wildlife. Escalating population growth and increasing demands for these multiple water uses make water availability, now measured in terms of quantity and quality, even more critical to the long-term sustainability of our communities and ecosystems.

The USGS implemented the National Water-Quality Assessment (NAWQA) program to support national, regional, and local information needs and decisions related to water-quality management and policy. Shaped by and coordinated with ongoing efforts of other Federal, State, and local agencies, the NAWQA Program is designed to answer: What is the condition of our Nation's streams and ground water? How are the conditions changing over time? How do natural features and human activities affect the quality of streams and ground water, and where are those effects most pronounced? By combining information on water chemistry, physical characteristics, stream habitat, and aquatic life, the NAWQA Program aims to provide science-based insights for current and emerging water issues. Program results can contribute to informed decisions that result in practical and effective waterresource management and strategies that protect and restore water quality.

Since 1991, the NAWQA Program has implemented interdisciplinary assessments in more than 50 of the Nation's most important river basins and aquifers, referred to as "study units." Collectively, these study units account for more than 60 percent of the overall water use and population served by public-water supply, and are representative of the Nation's major hydrologic landscapes, priority ecological resources, and agricultural, urban, and natural sources of contamination.

Each assessment is guided by a nationally consistent study design and methods of sampling and analysis. The assessments thereby build local knowledge about water-quality issues and trends in a particular stream or aquifer while providing an understanding of how and why water quality varies regionally and nationally. The consistent, multiscale approach helps to determine if certain types of water-quality issues are isolated or pervasive, and allows direct comparisons of how human activities and natural processes affect water quality and ecological health in the Nation's diverse geographic and environmental settings. Comprehensive assessments on pesticides, nutrients, volatile organic compounds, trace metals, and aquatic ecology are developed at the national scale through comparative analyses of the study-unit findings.

The USGS places high value on the communication and dissemination of credible, timely, and relevant science so that the most recent and available knowledge about water resources can be applied in management and policy decisions. We hope this NAWQA publication will provide you the needed insights and information to meet your needs, and thereby foster increased awareness and involvement in the protection and restoration of our Nation's waters.

The NAWQA Program recognizes that a national assessment by a single program cannot address all water-resource issues of interest. External coordination at all levels is critical for a fully integrated understanding of watersheds and for cost-effective management, regulation, and conservation of our Nation's water resources. The program, therefore, depends extensively on the advice, cooperation, and information from other Federal, State, interstate, tribal, and local agencies, nongovernment organizations, industry, academia, and other stakeholder groups. The assistance and suggestions of all are greatly appreciated.

Robert M. Hirsch

Associate Director for Water 
IV Contents 


\section{CONTENTS}

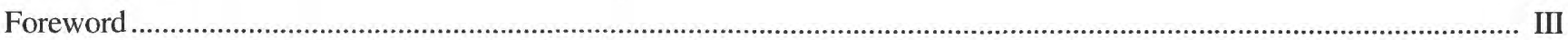

Abstract

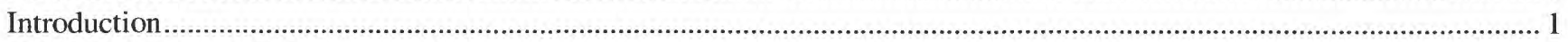

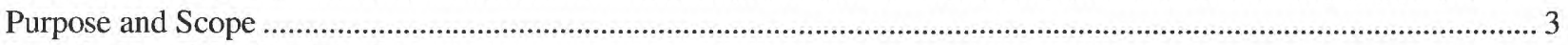

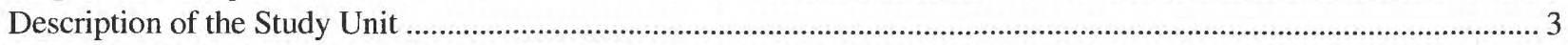

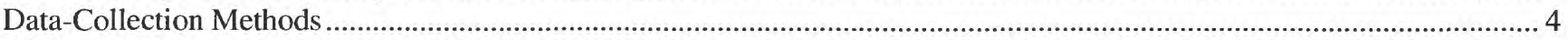

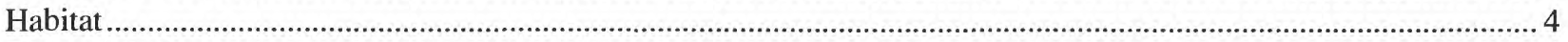

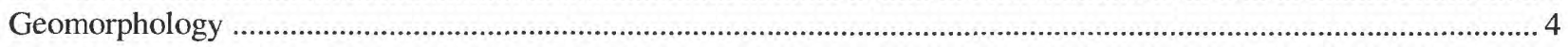

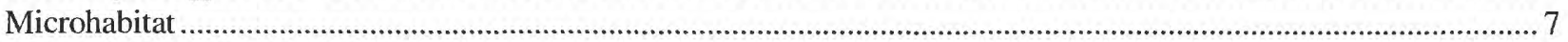

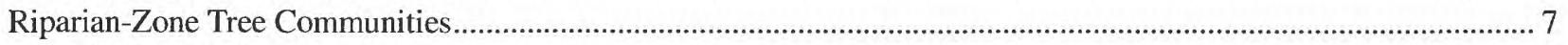

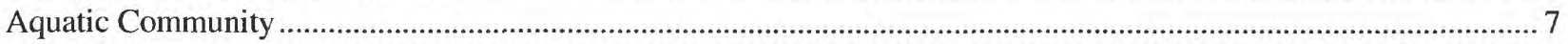

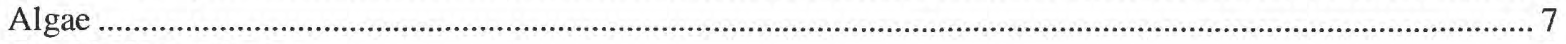

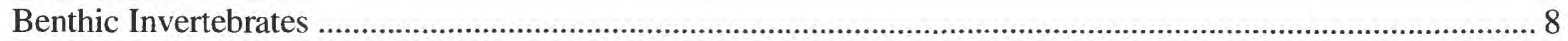

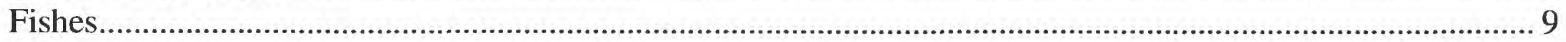

Summary …1-1.

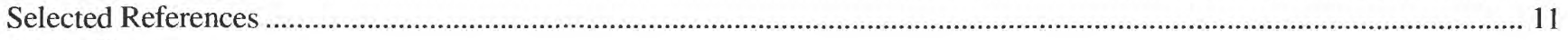

Appendixes

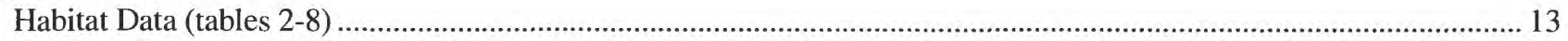

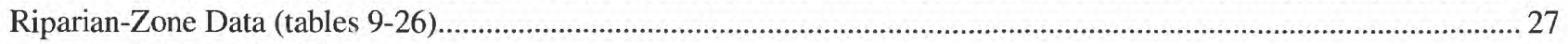

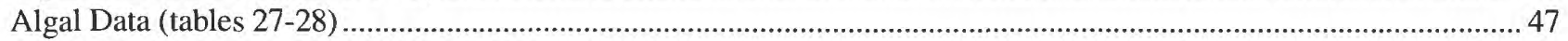

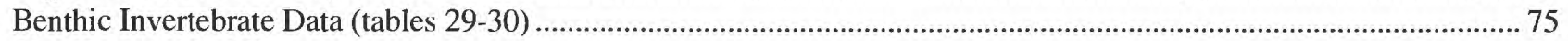

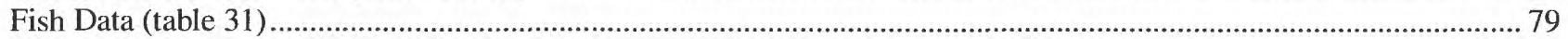

\section{FIGURES}

1. Map showing location of the Santee River Basin and Coastal Drainages study unit and ecological sampling sites,

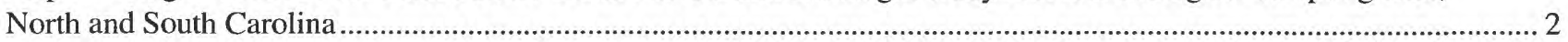

2. Map showing location of the National Water-Quality Assessment Program study units in the United States .................... 3

3. Stylized stream sketch showing plan and cross-section view collection points for stream habitat data ............................. 5

4. Bar chart showing number of benthic invertebrate taxa identified in each stream reach of the Santee River Basin and Coastal Drainages study unit, North and South Carolina, 1996-98 ................................................................... 10

5. Bar chart showing number of fish species identified in each stream of the Santee River Basin and Coastal Drainages study unit, North and South Carolina, 1996-98.

\section{TABLES}

1. Data-collection and sampling sites in the Santee River Basin and Coastal Drainages study unit, North and South Carolina

2. Habitat characteristics of Jacob Fork near Ramsey, N.C., USGS 02143040; Indian Creek near Laboratory, N.C., USGS 02143500; and Brushy Creek near Pelham, S.C., USGS 021603257 .......................................................... 14

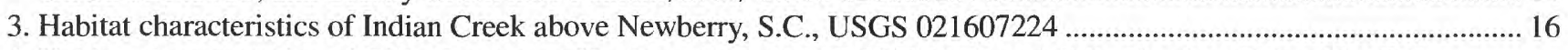

4. Habitat characteristics of Gills Creek near Hopkins, S.C., USGS 02169595, and Gills Creek at Columbia, S.C., USGS 02169570.

5. Habitat characteristics of Myers Creek near Hopkins, S.C., USGS 02169660; Cedar Creek below Myers Creek near Hopkins, S.C., USGS 02169670; and Cedar Creek at Cedar Creek Hunt Club near Gadsden, S.C., USGS 02169672 ... 20

6. Habitat characteristics of Toms Creek near Gadsden, S.C., USGS 021696966 and McTier Creek near Monetta, S.C., USGS 02172300 .

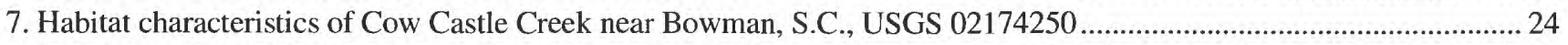

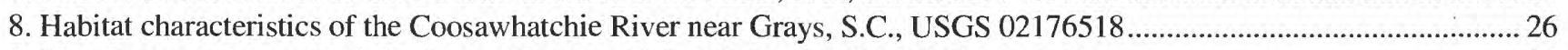

9. Riparian-zone trees, Jacob Fork near Ramsey, N.C., USGS site 02143040 (reach 1) …............................................. 28 
10. Riparian-zone trees, Indian Creek near Laboratory, N.C., USGS 02143500 (reach 2) ….............................................29

11. Riparian-zone trees, Brushy Creek near Pelham, S.C., USGS 021603257 (reach 4) ….................................................30

12. Riparian-zone trees, Indian Creek above Newberry, S.C., USGS 021607224 (reach 5) ............................................... 31

13. Riparian-zone trees, Indian Creek above Newberry, S.C., USGS 021607224 (reach 6) ..............................................32

14. Riparian-zone trees, Indian Creek above Newberry, S.C., USGS 021607224 (reach 7) ..............................................33

15. Riparian-zone trees, Gills Creek near Hopkins, S.C., USGS 02169595 (reach 8).......................................................34

16. Riparian-zone trees, Gills Creek at Columbia, S.C.. USGS 02169570 (reach 9) …......................................................35

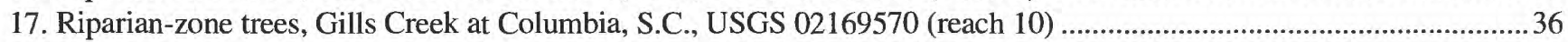

18. Riparian-zone trees, Myers Creek near Hopkins, S.C., USGS 02169660 (reach 11) ..................................................37

19. Riparian-zone trees, Cedar Creek below Myers Creek near Hopkins, S.C., USGS 02169670 (reach 12) ........................38

20. Riparian-zone trees, Cedar Creek at Cedar Creek Hunt Club near Gadsden, S.C., USGS 02169672 (reach 13)..............39

21. Riparian-zone trees, Toms Creek near Gadsden, S.C., USGS 021696966 (reach 14) ...................................................40

22. Riparian-zone trees, McTier Creek near Monetta, S.C., USGS 02172300 (reach 15) .................................................41

23. Riparian-zone trees, Cow Castle Creek near Bowman, S.C., USGS 02174250 (reach 16) ...........................................42

24. Riparian-zone trees, Cow Castle Creek near Bowman, S.C., USGS 02174250 (reach 17) ….......................................43

25. Riparian-zone trees, Cow Castle Creek near Bowman, S.C., USGS 02174250 (reach 18) ...........................................44

26. Riparian-zone trees, Coosawhatchie River near Grays, S.C., USGS 02176518 (reach 19).........................................45

27. Algal taxa and their maximum relative abundance in quantitative samples collected in the Santee River Basin

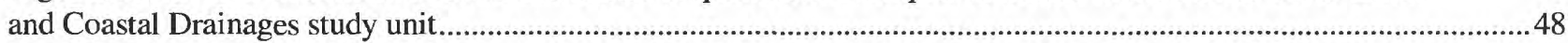

28. Algal cell densities in selected stream reaches in the Santee River and Coastal Drainages study unit ............................60

29. Benthic invertebrate density and diversity in the Santee River Basin and Coastal Drainages study unit.........................76

30. Benthic invertebrate species and density in streams in the Santee River Basin and Coastal Drainages study unit...........77

31. Fish species collected in streams of the Santee River Basin and Coastal Drainages study unit......................................... 80

Conversion Factors

\begin{tabular}{|rcl|}
\hline Multiply & By & To obtain \\
\hline centimeter $(\mathrm{cm})$ & 0.3937 & inch \\
square centimeter $\left(\mathrm{cm}^{2}\right)$ & 0.001076 & square foot \\
meter $(\mathrm{m})$ & 3.281 & foot \\
square meter $\left(\mathrm{m}^{2}\right)$ & 10.76 & square foot \\
square kilometer $\left(\mathrm{km}^{2}\right)$ & 0.3861 & square mile
\end{tabular}

\section{Additional Abbreviations and Acronyms}

\begin{tabular}{r|l}
\hline $\mathrm{mL}$ & milliliter \\
$\mu \mathrm{m}$ & micrograms per liter \\
$<$ & less than \\
$>$ & greater than \\
\hline ANSP & Academy of Natural Sciences (Philadelphia) \\
DTH & depositional targeted habitat \\
NAWQA & National Water-Quality Assessment program \\
QMH & qualitative multihabitat \\
RTH & richest targeted habitat \\
SANT & Santee River Basin and Coastal Drainages study unit \\
USGS & U.S. Geological Survey
\end{tabular}




\title{
Ecological Data Collected in the Santee River Basin and Coastal Drainages, North and South Carolina, 1996-98
}

\author{
By T.A. Abrahamsen
}

\author{
ABSTRACT \\ As part of the National Water-Quality \\ Assessment Program, ecological investigations \\ were conducted in 23 reaches of 16 streams in the \\ Santee River Basin and Coastal Drainages study \\ unit in North and South Carolina during 1996-98. \\ Habitat characteristics, such as stream width and \\ depth, bank composition, bank vegetative cover, \\ stream shading by overhanging vegetation, and \\ streambed composition were recorded. Algal and \\ benthic invertebrate communities were sampled \\ using quantitative and qualitative techniques. \\ These data will provide information needed to: \\ (1) support findings of the effects of human land- \\ use activities on water quality by augmenting or \\ enhancing physical and chemical water-quality \\ data, (2) provide a basic overview of aquatic \\ community structure in selected stream reaches in \\ the study unit, and (3) provide a means for \\ comparing aquatic communities in subsequent \\ years of the assessment program.
}

\section{INTRODUCTION}

During 1996-98, ecological data were collected in the Santee River Basin and Coastal Drainages (SANT) study unit (fig. 1) as part of the U.S. Geological Survey (USGS) National Water-Quality Assessment (NAWQA) Program. An objective of this ongoing investigation is an attempt to integrate ecological data with more traditional chemical and physical methods of determining the quality of water. These data were collected coincidentally with surface-waterquality data (Maluk, 2000), bed-sediment and tissue data (Abrahamsen, 1999), and ground-water-quality data as part of the comprehensive investigation within the study unit (Hughes and others, 2000).

The NAWQA Program is designed to evaluate the quality of the Nation's water resources and to link an assessment of the status and trends in water quality with an understanding of the natural and human factors that affect the quality of water. That assessment is based on a nationally consistent design structure that incorporates a multiscale, interdisciplinary approach for ground-water and surface-water investigations. The goal of the program is to provide an understanding of the causes of processes that affect water quality at various spatial and temporal scales, so that knowledge 


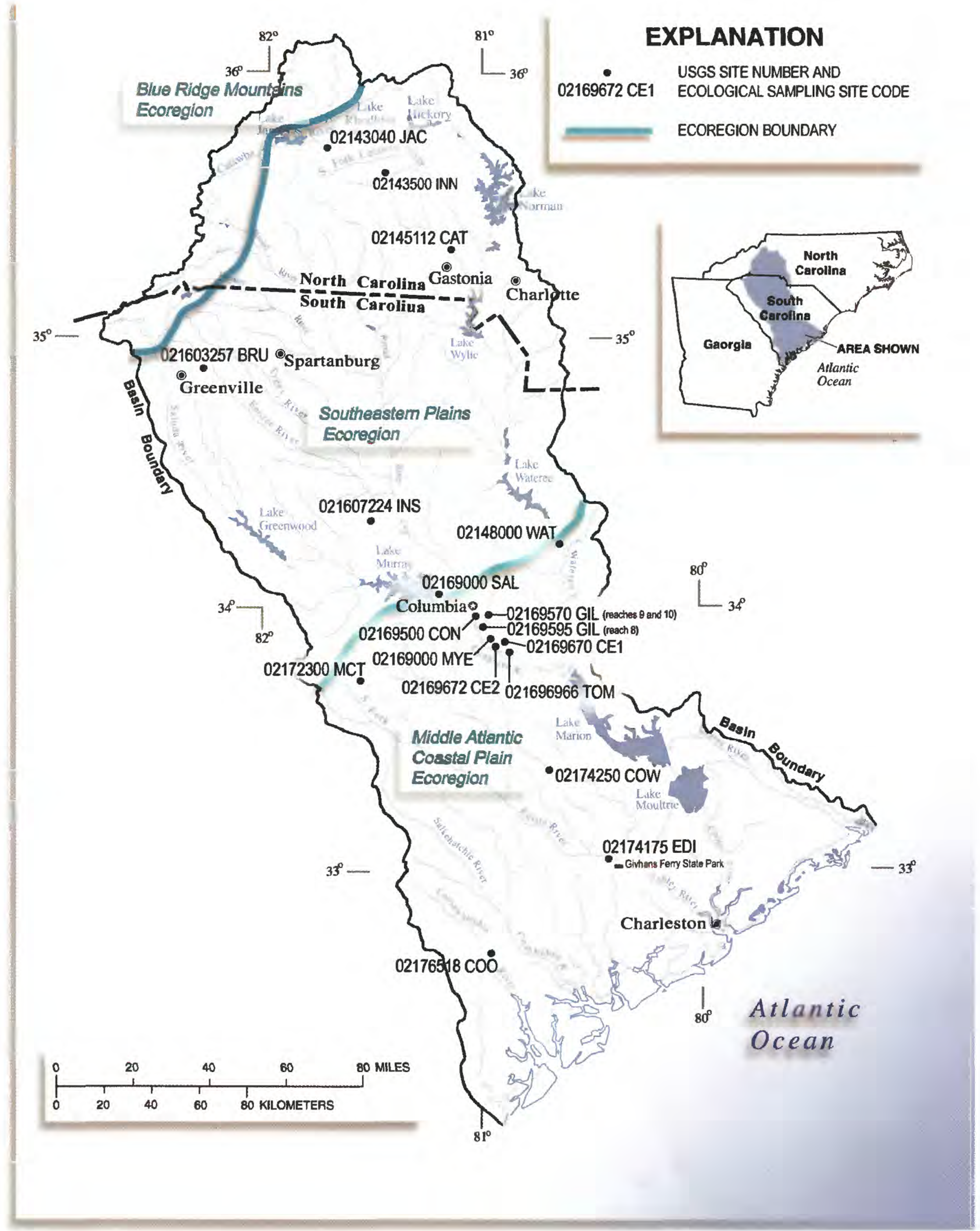

Figure 1. Location of the Santee River Basin and Coastal Drainages (SANT) study unit and ecological sampling sites, North and South Carolina. 
can be useful in policy and management actions that improve water quality. The program integrates information about water quality for a wide range of spatial scales, locally and nationally. For example, it focuses on water-quality conditions, such as organic compounds and trace elements in water associated with urban areas, and nutrients and pesticide concentrations in water associated with large agricultural regions.

The building blocks of the NAWQA Program are study-unit investigations in 51 major hydrologic basins in the United States (Leahy and others, 1990). These study units cover about one-half of the area of the country (fig. 2) and encompass 60 to 70 percent of the public water supplies. They include hydrologic systems having diverse human and natural factors that can affect water quality. The SANT is one of these study units.

\section{Purpose and Scope}

Ecological data, collected as part of the NAWQA Program in the SANT study unit (fig. 1) during 1996-98, are presented in this report. The data describe ecological characteristics in 23 stream reaches within 16 different streams (table 1). Habitat measurements were made in 16 categories that include stream-channel geomorphology, substrate composition, and water-column characteristics (tables 2-8). Riparian-zone tree species were identified and measured (tables 9-26). Aquatic community measurements involved qualitative and quantitative collections of algae (tables 27-28), benthic invertebrates (tables 29-30), and fishes (table 31 ) from a variety of habitats. Tables 2-31 are presented as appendixes. Descriptions of data-collection methods and explanations of pertinent reach characteristics are provided in the text.

\section{Description of the Study Unit}

The SANT study unit (fig. 1) encompasses approximately 61,100 square kilometers $\left(\mathrm{km}^{2}\right)$ and contains parts of western North Carolina and much of South Carolina. Sections of three ecoregions-the Blue Ridge, the Southeastern Plains, and the Middle Atlantic Coastal Plain (Omernik, 1987, 1995)-are included within the study unit. Major streams in the study unit include the: Saluda, Broad, Congaree, Catawba, and Wateree Rivers. The Saluda and Broad Rivers converge to form the Congaree River, at Columbia, S.C. The Catawba River (with headwaters in North Carolina) becomes the Wateree River in S.C., downstream of Lake Wateree. The Wateree and Congaree Rivers converge to form the Santee River which then forms Lakes Marion and Moultrie before flowing to the coast and into the Atlantic Ocean. In addition, McTier and Cow Castle Creeks and the Edisto and Coosawhatchie Rivers (unconnected to the Santee River Basin), contain ecological sampling sites that were used for this study (fig. 1). A more detailed description of the study unit is provided in Abrahamsen and others (1997).

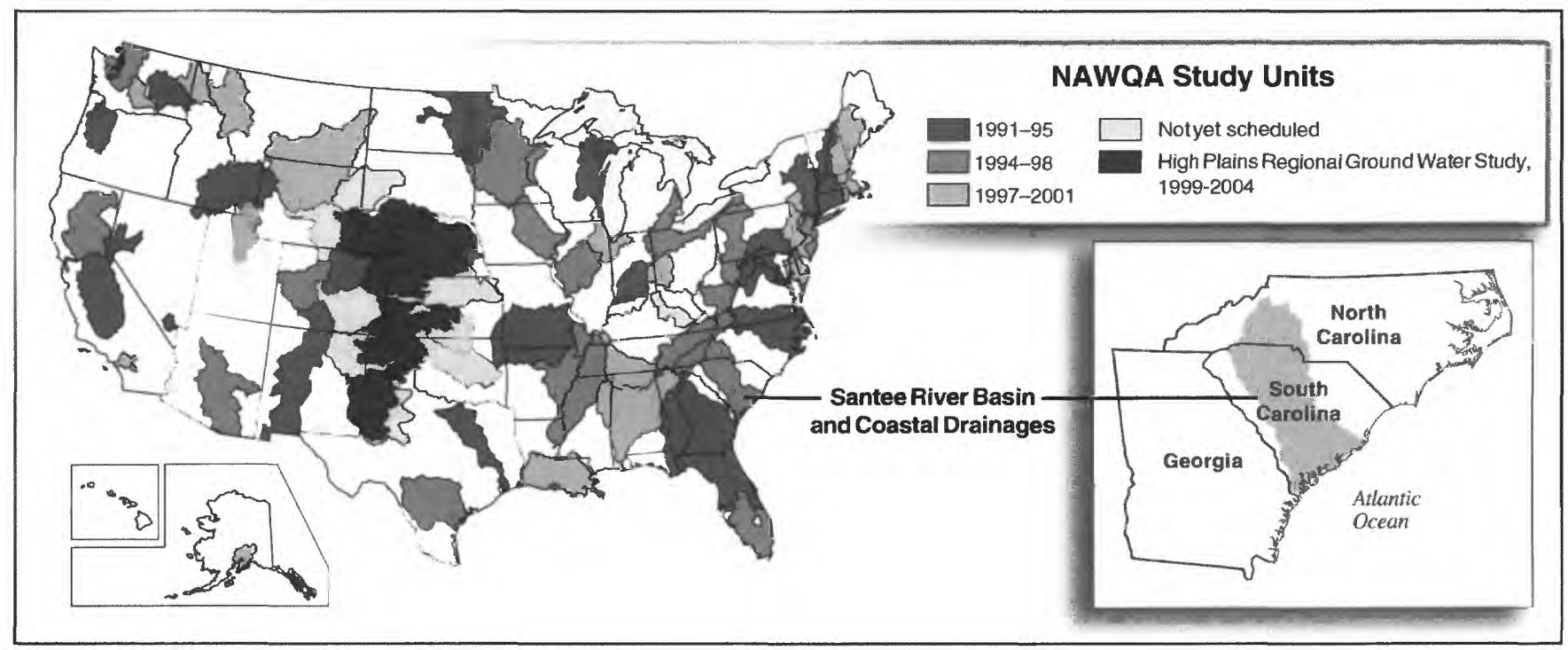

Figure 2. Location of the National Water-Quality Assessment (NAWQA) Program study units in the United States. 
Table 1. Data-collection and sampling sites in the Santee River Basin and Coastal Drainages study unit, North and South Carolina [km², square kilometers; ', degrees; ', minutes; ", seconds; CR, county road]

\begin{tabular}{|c|c|c|c|c|c|c|}
\hline $\begin{array}{l}\text { U.S. Geological Survey site name } \\
\text { and number }\end{array}$ & $\begin{array}{l}\text { Reach } \\
\text { ID }\end{array}$ & $\begin{array}{l}\text { Reach } \\
\text { number }\end{array}$ & $\begin{array}{c}\text { Basin } \\
\text { area } \\
\left(\mathrm{km}^{2}\right)\end{array}$ & $\begin{array}{l}\text { Lati- } \\
\text { tude }\end{array}$ & $\begin{array}{l}\text { Longi- } \\
\text { tude }\end{array}$ & $\begin{array}{l}\text { Reference site } \\
\text { description/location }\end{array}$ \\
\hline \multicolumn{7}{|l|}{ North Carolina sites: } \\
\hline 02143040 Jacob Fork near Ramsey & JAC & 1 & 67 & $35^{\circ} 35^{\prime} 26^{\prime \prime}$ & $81^{\circ} 34^{\prime} 02^{\prime \prime}$ & Upstream center of bridge on Old NC-18 \\
\hline 02143500 Indian Creek near Laboratory & INN & 2 & 168 & $35^{\circ} 25^{\prime} 46^{\prime \prime}$ & $81^{\circ} 17^{\prime} 24^{\prime \prime}$ & Upstream center of bridge on Laboratory Rd. \\
\hline $\begin{array}{l}02145112 \text { South Fork Catawba River at } \\
\text { McAdenville }^{2}\end{array}$ & CAT & 3 & 1,630 & $35^{\circ} 17^{\prime} 10^{\prime \prime}$ & $81^{\circ} 06^{\prime} 00^{\prime \prime}$ & $\begin{array}{l}\text { Upstream center of bridge on Spencer } \\
\text { Mountain Rd. }\end{array}$ \\
\hline \multicolumn{7}{|l|}{ South Carolina sites: } \\
\hline 021603257 Brushy Creek near Pelham & BRU & 4 & 36 & $34^{\circ} 51^{\prime} 48^{\prime \prime}$ & $82^{\circ} 15^{\prime} 06^{\prime \prime}$ & Upstream center of bridge on Pelham Rd. \\
\hline 021607224 Indian Creek above Newberry & INS & $5,6,7$ & 130 & $34^{\circ} 25^{\prime} 50^{\prime \prime}$ & $81^{\circ} 36^{\prime} 30^{\prime \prime}$ & Upstream center of bridge on Monument Rd. \\
\hline 02169595 Gills Creek near Hopkins & GIL & 8 & ${ }^{3}>155$ & $33^{\circ} 55^{\prime} 22^{\prime \prime}$ & $80^{\circ} 58^{\prime} 58^{\prime \prime}$ & USGS gage 02169570 \\
\hline 02169570 Gills Creek at Columbia & GIL & 9,10 & 155 & $33^{\circ} 59^{\prime} 22^{\prime \prime}$ & $80^{\circ} 58^{\prime} 28^{\prime \prime}$ & USGS gage 02169570 \\
\hline 02169660 Myers Creek near Hopkins & MYE & 11 & 83 & $33^{\circ} 50^{\prime} 27^{\prime \prime}$ & $80^{\circ} 51^{\prime} 36^{\prime \prime}$ & $\begin{array}{l}\text { Upstream center of bridge that crosses the } \\
\text { stream on Richland CR-734 }\end{array}$ \\
\hline $\begin{array}{l}02169670 \text { Cedar Creek below Myers Creek } \\
\text { near Hopkins }\end{array}$ & CEl & 12 & 52 & $33^{\circ} 50^{\prime} 20^{\prime \prime}$ & $80^{\circ} 50^{\prime} 45^{\prime \prime}$ & $\begin{array}{l}\text { Upstream center of bridge that crosses the } \\
\text { stream on Richland CR-734 }\end{array}$ \\
\hline $\begin{array}{l}\text { 02169672 Cedar Creek at Cedar Creek Hunt } \\
\text { Club near Gadsden }\end{array}$ & CE2 & 13 & 184 & $33^{\circ} 48^{\prime} 58^{\prime \prime}$ & $80^{\circ} 49^{\prime} 39^{\prime \prime}$ & USGS gage 02169672 \\
\hline 021696966 Toms Creek near Gadsden & TOM & 14 & 101 & $33^{\circ} 48^{\prime} 41^{\prime \prime}$ & $80^{\circ} 43^{\prime} 30^{\prime \prime}$ & $\begin{array}{l}\text { Upstream end of culvert under the road } \\
\text { crossing the stream }\end{array}$ \\
\hline 02172300 McTier Creek near Monetta & MCT & 15 & 47 & $33^{\circ} 45^{\prime} 15^{\prime \prime}$ & $81^{\circ} 36^{\prime} 00^{\prime \prime}$ & USGS gage 02172300 \\
\hline 02174250 Cow Castle Creek near Bowman & COW & $16,17,18$ & 61 & $33^{\circ} 22^{\prime} 31^{\prime \prime}$ & $80^{\circ} 42^{\prime} 00^{\prime \prime}$ & USGS gage 02174250 \\
\hline 02176518 Coosawhatchie River near Grays & $\mathrm{COO}$ & 19 & 1,040 & $32^{\circ} 38^{\prime} 35^{\prime \prime}$ & $80^{\circ} 57^{\prime} 35^{\prime \prime}$ & USGS gage 02176518 \\
\hline 02148000 Wateree River near Camden ${ }^{2}$ & WAT & 20 & 13,100 & $34^{\circ} 14^{\prime} 42^{\prime \prime}$ & $80^{\circ} 39^{\prime} 15^{\prime \prime}$ & $\begin{array}{l}\text { Upstream center of bridge on US- } 601 \text { and } \\
\text { SC-34 }\end{array}$ \\
\hline 02169000 Saluda River near Columbia ${ }^{2}$ & SAL & 21 & 6,530 & $34^{\circ} 00^{\prime} 50^{\prime \prime}$ & $81^{\circ} 05^{\prime} 17^{\prime \prime}$ & USGS gage 02169000 \\
\hline 02174175 Edisto River near Cottageville ${ }^{2}$ & EDI & 22 & 7,080 & $33^{\circ} 03^{\prime} 20^{\prime \prime}$ & $80^{\circ} 27^{\prime} 00^{\prime \prime}$ & $\begin{array}{l}\text { Mas Old Field Boat Landing off SC } 61 \text {, } \\
\text { upstream of Givhans }\end{array}$ \\
\hline 02169500 Congaree River at Columbia ${ }^{2}$ & $\mathrm{CON}$ & 23 & 20,300 & $33^{\circ} 59^{\prime} 35^{\prime \prime}$ & $81^{\circ} 03^{\prime} 00^{\prime \prime}$ & $\begin{array}{l}\text { Barney Jordan Public Boat Landing off } \\
\text { SC-48 in Southeastern Columbia }\end{array}$ \\
\hline
\end{tabular}

\section{DATA-COLLECTION METHODS}

Field-collection and assessment methods used in this study followed standard NAWQA protocols, referenced below. Unless otherwise noted, there was no deviation from the protocols.

\section{Habitat}

Habitat data were collected in accordance with Meador and others (1993a). A revised habitat protocol (Fitzpatrick and others, 1998) was published subsequent to the collection of data reported herein.
Where practicable, the data were adjusted to coincide with the requirements of the new protocol to make them consistent with data collected in later NAWQA studies.

\section{Geomorphology}

Reaches (stream sections), ranging from 150 meters $(\mathrm{m})$ in small streams to $2,000 \mathrm{~m}$ in rivers, were the principal sampling sites for the collection of ecological data. One or more reaches were selected for description of habitat characteristics at each of 12 wadeable streams. Three reaches were sampled in each 
of three streams to investigate spatial variability within streams. Several reaches in the study unit coincided with surface-water-quality sampling sites, so watercolumn chemistry data were available for comparison with ecological information.

Six transects, oriented perpendicular to streamflow, were measured along each reach The first and last transects defined the start and end, respectively, of each reach. Transects were laid out at approximately equidistant intervals and a number of physical attributes were measured at each (tables 2-8). Characteristics of at least seven points along each transect were measured or observed and noted (fig. 3).

Figure 3. Plan and cross-section view coliection points for stream habitat data.
The points included the left and right bankfull points, the left and right edges of the water, and three or more points in the stream (labeled points $1,2,3, \ldots$ in the tables) that corresponded to the thalweg (defined as the
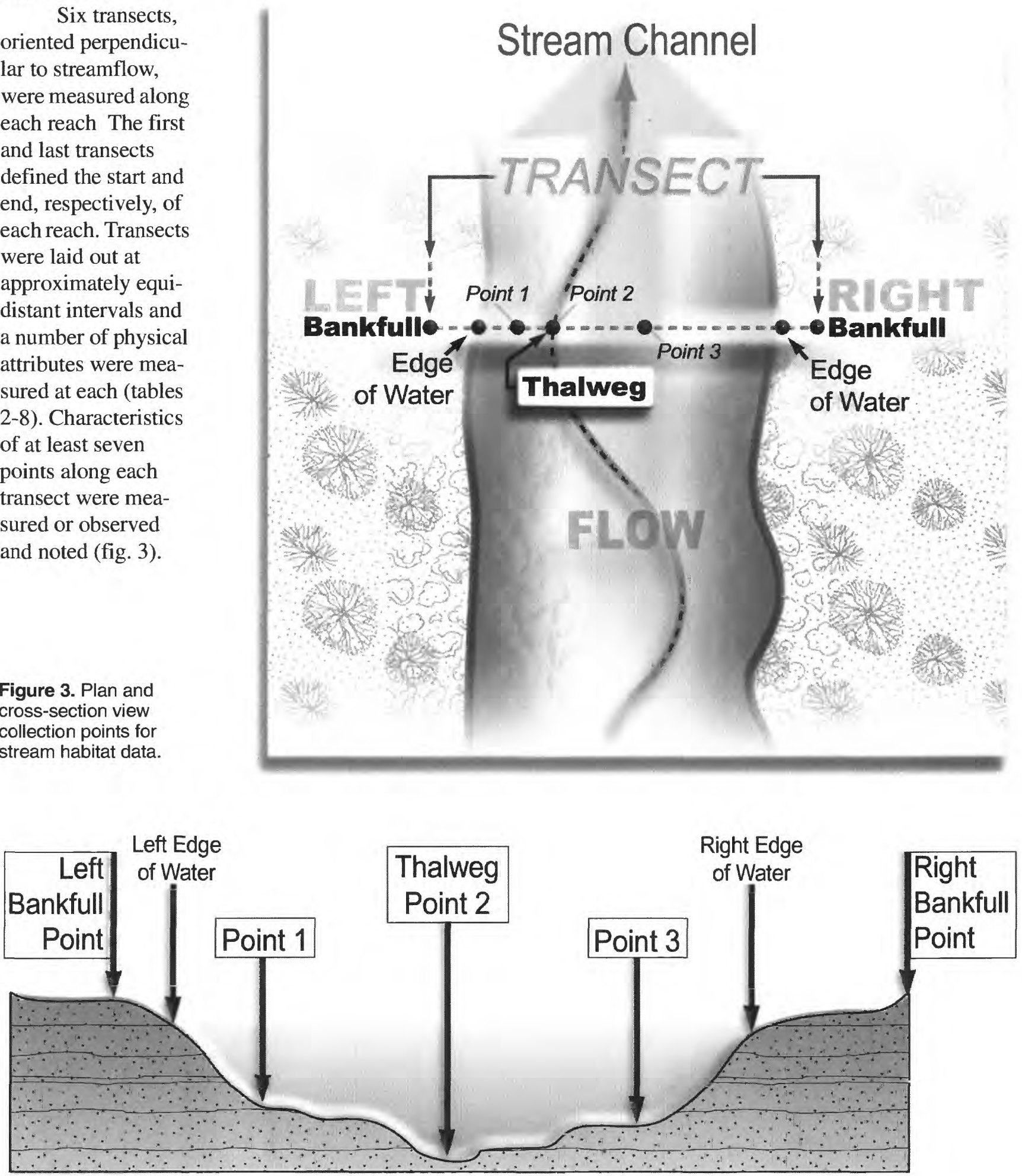
deepest part of the channel), and to one location on each side of the thalweg, halfway between it and the edge of the water. If no thalweg existed, the points were spaced equidistantly across the channel.

The characteristics (modified from Fitzpatrick and others, 1998) measured at each transect included:

Distance from the reach boundary closest to the reference location.- This distance was measured as the distance along the stream channel from a transect to the reach boundary closest to the reference location.

A reference location is a permanent structure that is expected to remain in place for several years, and from which the study reach can be readily located.

Channel width at bankfull.-The bankfull points are those points on either side of a stream where water flows onto a floodplain and is no longer contained by the stream channel. The channel width is the distance between the two bankfull points. For example, referring to figure 3 , the channel width at bankfull would be measured as the distance between the points represented by the two vertical lines, labeled left or right “...bankfull point" on either side of the stream.

Bank width.-Banks include geomorphic features, such as channel shelves, and are flooded, on average, more than once every 1 to 3 years (Hupp, 1986). Banks are bounded by the channelbed and the floodplain. Thus, bank width is the distance between the channelbed and the floodplain. This distance is measured with a tape measure or rangefinder (Meador and others, 1993b).

Floodplain width.-The floodplain is a relatively flat surface that is flooded, on average, once every 1 to 3 years (Leopold and others, 1964). The floodplain is bounded by features at higher elevations, such as terraces, that are flooded, on average, once every 3 or more years (Hupp, 1986). If the floodplain width was less than $(<) 50 \mathrm{~m}$, it was measured with a tape measure or rangefinder. Floodplain widths greater than (>) $50 \mathrm{~m}$ were determined by observation or from maps or aerial photographs, and are indicated as $>50 \mathrm{~m}$ in the tables.

Depth.-The depth of the water was measured at each of the designated points in the water along the transect by using a meterstick, wading rod, or a surveyor's transit and rod.
Distance from left bank.-The distance of each point from the left bankfull point of the transect was measured by using a tape measure, range finder, or a surveyor's transit and rod.

Streambed substrate composition.-The composition of the substrate was determined by observation and a descriptive code was assigned for each point. The descriptive codes are defined in the headnotes of tables 2-8.

Silt.-The presence or absence of silt was recorded at each point within the stream. The presence of silt is indicative of the velocity and flow of the water at that point. Large deposits of silt indicate the water velocity is too low to carry suspended sediments or resuspend sediments that have been deposited.

Embeddedness. - This measure refers to the extent that coarse material (boulders, cobbles, or gravel) is surrounded or covered by sand or finer sediment . As the percentage of embeddedness decreases, biotic productivity is thought to decrease (Platts and others, 1983). Embeddedness was visually estimated and rated by the percentage of surface area of large particles covered by fine sediment, as listed below:

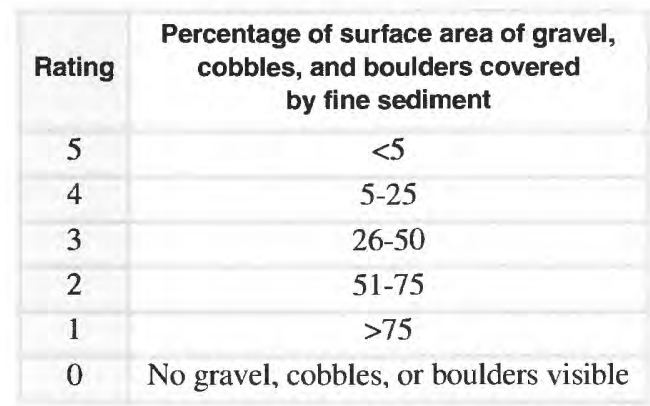

Canopy angle.-The amount of direct sunlight that a unit area receives is an important factor in the composition of the biological community. Canopy angle provides a measure of the openness of the vegetation overhanging the stream, or the extent to which manmade structures shade the stream. A clinometer was used to determine the angles from the line of sight of the investigator (while standing in midchannel) to the tallest structure (for example, tree, shrub, building, or grass) on the left and right banks. The canopy angle can be used to determine the amount of direct sunlight potentially reaching the stream. The sum of the canopy angles was subtracted from 180 degrees $\left({ }^{\circ}\right)$, resulting in the open-canopy angle. The smaller the open-canopy angle, the more shading of the stream at that point. 
Aspect.-This characteristic is the direction of the flow of the stream $\left(0-360^{\circ}\right)$. It was measured from the middle of the stream channel at the transect, by facing downstream and reading a compass pointed parallel to streamflow.

Habitat features.-Any mineral or organic matter that produces shelter for aquatic organisms to rest, hide, or feed, is considered to be habitat. Habitat includes the natural features of a stream, such as boulders, woody debris, and undercut banks, as well as aquatic macrophyte beds and artificial structures, such as discarded tires, appliances, or parts of automobiles. The type and amount (as a percentage) of all habitat features were measured that were partly or wholly within a 2-m zone on either side of each transect. Habitat features were not counted when they were at insufficient depth (usually $<20$ centimeters $(\mathrm{cm})$ ). For example, an accumulation of woody debris in $5 \mathrm{~cm}$ of water was not considered a significant habitat feature. Habitat-feature type was noted using the letter codes listed in the table headnotes.

Bar/island.-Some features of a stream channel can be indicative of the velocity and flow of water. Bars generally consist of sand, gravel, and cobbles, typically have no woody vegetation, and are transitory in nature. Islands are classified as bars in midchannel with permanent woody vegetation. On average, bars and islands are flooded once per year.

Bank height.-This is the vertical distance between the thalweg and the bankfull discharge point at the transect being measured.

Bank erosion.-A visual determination of the presence or absence of bank erosion was recorded for each end of each transect.

Streambank dominant substrate.-The same codes used for the bed substrate description were used for streambank dominant substrate. Data listed herein follow the protocol by Meador and others (1993b).

\section{Microhabitat}

Microhabitat is the localized set of conditions that describe where aquatic organisms live. It consists of relatively homogeneous patches of macrophytes, woody debris, or bed substrate within areas such as pools, riffles, and runs, characterized by channel shape and stream velocity (Fitzpatrick and others, 1998).

Studies have revealed that analyzing relational patterns between aquatic organisms and habitat at the microhabitat scale can provide insight to relational patterns between biota and habitat at larger scales (Hawkins, 1985; Biggs and others, 1990).
Collection of microhabitat data is essential to understanding the relations between benthic invertebrate and algal communities, and the physical, chemical, and biological factors affecting them. Invertebrate and algal samples were collected at specific locations within geomorphic channel units in a reach. Conversely, fishes were collected throughout each reach because they generally are not associated with a specific microhabitat. Procedures for collecting microhabitat data are described in Cuffney and others, 1993 and Porter and others, 1993.

\section{Riparian-Zone Tree Communities}

A point-centered quarter method (MuellerDombois and Ellenberg, 1974) was used to assess the nature of the riparian-zone tree communities. This method allows a quantitative estimate of tree density and biomass and provides a record of the tree species. A pivot point was randomly selected at each end of a transect. From the pivot point, the nearest tree in each quarter section $\left(90^{\circ}\right.$ section of a $360^{\circ}$ circle) was identified. Distance from the pivot point and the diameter of each tree at chest height were measured, and the scientific and common names of the trees were recorded (see tables 9-26). As many as 48 trees were recorded at each of 18 reaches. At some sites there were no riparian-zone trees or the riparian zone was inaccessible.The riparian zones of the 6 river reaches were not assessed.

\section{Aquatic Community}

The aquatic community, as defined for this report, includes multicellular and single-celled algae (diatoms), benthic invertebrates, and fishes.

\section{Algae}

Algal samples were collected using procedures outlined in Porter and others (1993). For quantitative analysis and taxonomic enumeration, two types of discrete areal samples were collected, depending on the habitat being sampled. The faunistically richest habitat, called the richest targeted habitat (RTH), and the depositional targeted habitat (DTH) in bed sediments were sampled by semiquantitative methods to determine organism density and diversity.

The RTH in 21 of the reaches in the study area included logs and other woody snags. In 2 reaches the RTH consisted of rocks and gravel. For woody surfaces 
and rocks, the branch or rock was gently moved to within about $3 \mathrm{~cm}$ of the water surface and the algal sample was collected in place, with the SG-92 sampling device as described below. Alternatively, the branch or rock was slowly removed from the water (to reduce loss of algal cells through runoff) and placed in a large plastic dishpan for processing.

A modified syringe sampling device (SG-92), designed for the NAWQA Program (Porter and others, 1993) was used for collecting algae from the surfaces of branches and rocks. This device is a 5-cm-long plastic cylinder, with an inside diameter of $2.54 \mathrm{~cm}$, and a rubber O-ring attached to one end. To collect algal samples, the SG-92 was held tightly against the surface of the branch or rock to make a watertight seal with the O-ring. While the SG-92 was held in place, a stiff-bristled brush was used to thoroughly scrub the area within the O-ring. A plastic tube attached to a 60-milliliter $(\mathrm{mL})$ syringe was inserted into the cylinder and the water containing the algae was withdrawn into the syringe. The contents of the syringe were placed in a collection jar. During the removal process, the SG-92 was maintained in position on the branch or rock so that a second collection from the same area could be accomplished. For the second collection, a small volume of distilled water was placed into the cylinder and the scrubbing and withdrawal process was repeated. The result was a collection of algae from a discrete area of $5.07 \mathrm{~cm}^{2}$ of the branch or rock. The process was completed for at least two sites within each of five locations in the reach being studied, resulting in a composite sample from at least $51 \mathrm{~cm}^{2}$.

Collection of algal samples from bed sediments (DTH) was accomplished with a shallow plastic petridish cover with an inside diameter of $4.9 \mathrm{~cm}$ and a spatula. The petri dish was inverted under water to remove air and carefully pushed into the surface of the bed sediment. While holding the dish on the sediment with a finger, the spatula was carefully inserted under it to capture the sediment in the dish. Dish and spatula were removed from the water while the dish was held firmly against the spatula then inverted so that the dish opening was facing up. The spatula was removed and the material in the petri dish was scraped into a sample jar. This process was conducted at least twice at each of five sites within the reach, resulting in a composite sample of at least $77 \mathrm{~cm}^{2}$.
For taxonomic evaluation only, qualitative multihabitat $(\mathrm{QMH})$ samples of macroalgae and aquatic mosses were collected in each reach where these organisms were observed. Sampling was accomplished by hand and forceps, collecting visible algae and moss at several sites within each reach.

All algal samples were preserved in 3 to 5 percent buffered formaldehyde. The Academy of Natural Sciences in Philadelphia, Pennsylvania, was contracted to perform taxonomic analyses. Academy phycologists identified algal taxa in the quantitative samples from each reach (table 27)

\section{Benthic Invertebrates}

Benthic invertebrates were collected in RTH and QMH samples (no DTH sampling was required) using qualitative and quantitative techniques (Cuffney and others, 1993). The RTH in each reach (determined by observation to be either woody snags or rocks and gravel) was sampled in discrete areal collections. For example, submerged branches and sticks were detached and collected in an aquatic D-frame net. The branches were scrubbed with soft-bristled brushes and rinsed with streamwater into a bucket. All loose material on the branches was removed and the branches were picked apart to dislodge burrowing and hiding invertebrates. All debris and loose material were processed through a standard brass 425 -micrometer $(\mu \mathrm{m})$ mesh sieve. Large organic debris, such as leaves and twigs, were examined, and any adhering organisms or material were brushed into the sieve before the debris was discarded. Invertebrates and small organic debris retained by the sieve were placed in a sample container.

Two reaches in the study unit had designated RTHs of rocks and gravel. The collection procedure for this type of RTH is different from that used for a woody RTH. A Surber sampler (Cuffney and others, 1993) was used to collect benthic invertebrates at the reach in the South Fork Catawba River (USGS Site Number 02145112 ) This sampler is a $30.5 \mathrm{~cm}$-square frame with attached Nitex net having $425-\mu \mathrm{m}$ mesh openings. The sampler was held perpendicular to streamflow and pressed tightly to the stream bottom, so that the net was stretched out behind (downstream of) the frame by the current. The sampling area was delineated by an extendable frame attached to the net frame. The extendable frame encompasses $929 \mathrm{~cm}^{2}$. 
The Saluda River (USGS Site Number 02169000) reach was sampled with a Hess sampler (Cuffney and others, 1993). This device is an open-ended cylinder with an inner diameter of $33 \mathrm{~cm}$ and a sampling area of $855 \mathrm{~cm}^{2}$. It has a collection net ( $425-\mu \mathrm{m}$ mesh) attached to a hole in the side and has a screened ( $425-\mu \mathrm{m}$ mesh) opening opposite the collection net. Water flows through the screened opening and washes organisms into the collection net.

For the Surber and Hess samplers, rocks within the sampling area were carefully picked up, held in front of the net opening, and gently brushed with a fingernail brush or soft-bristled toothbrush so that adhering material was washed into the net by the current. Cleaned rocks were carefully examined for invertebrates and such organisms were removed from the rock surface with forceps and placed in a separate vial designated "large-rare" organisms.

Once the rocks had been cleaned and removed, the sample area was thoroughly dug up to a depth of $0.1 \mathrm{~m}$ with a marked metal bar. Where a depth of $0.1 \mathrm{~m}$ was not possible, the digging was done to the greatest depth practical. Final processing of the rock/gravel RTH samples was accomplished in the same manner as for those samples collected in the woody snag RTHs. Qualitative multihabitat (QMH) samples were collected from logs, branches, root masses, and rocks by using nets, forceps, and fingers, and by searching for individual organisms in leaves and organic detritus. All qualitative samples were processed through a standard brass $212-\mu \mathrm{m}$ mesh sieve. All retained material from the quantitative and qualitative samples was preserved in a 10 percent solution of buffered formaldehyde and sent to the USGS National Water Quality Laboratory in Lakewood, Colorado, for taxonomic evaluation.

The surface area for each collection from woody snags was calculated using the standard formula for determining the area of a cylinder (area $=\pi d h$, where $d=$ diameter, and $h=$ height or length), and the total area was used to determine the density of the benthic invertebrates at each site. The diversity of the benthic invertebrate community at each site was determined through a count of the total number of different species or higher taxa. Community diversity (total number of taxa) is used as an indicator of water quality. Several indices of diversity have been developed (Plafkin and others, 1989) and fine tuned. These indices concentrate on the total number of species, the number of species of a particular family or order, and the number of different feeding guilds (such as shredders or scrapers) represented by the organisms in a stream.
The density (number of organisms per unit area) of the invertebrate community also is indicative of the quality of the water and the type and quality of the habitat in a stream. Under stressful conditions, the more sensitive organisms undergo intense selection pressure. As they die out, the niches they vacate become available to more tolerant organisms that multiply because of the decrease in competition and the concomitant increase in available resources. Communities where only a few species hold numerical dominance are generally considered to have undergone some type of environmental stress. The number of taxa identified in each reach is shown in figure 4 . The diversity and density of the benthic invertebrate community at each site are presented in tables 29 and 30 .

\section{Fishes}

Fish collections were conducted in accordance with Meador and others (1993a). Fishes were collected by power-fishing techniques, using a backpack power fisher in most streams. In reach 8 (Gills Creek near Hopkins, S.C.) and reach 13 (Cedar Creek at Cedar Creek Hunt Club near Gadsden, S.C.), where water depth was too great for safe operation of the backpack unit, a commercial, barge-mounted power-fishing unit was used. A noncommercial, boat-mounted power-fishing apparatus was used to collect fishes at reach 19 (Coosawhatchie River near Grays, S.C.) and a second time at reach 13. In the Wateree, Saluda, Edisto, and Congaree Rivers (reaches $20,21,22$, and 23 , respectively), a 5.5-m boat, equipped with a commercial boat-mounted power-fishing apparatus was used to collect fishes.

In small, wadeable streams, three people (an operator and two collectors) worked as a team to collect fishes. Starting at the most downstream end of a reach, the team member with the backpack shocker proceeded upstream, followed by the collectors (carrying longhandled nets) wading behind and to each side. The team waded upstream from bank to bank, shocking and netting stunned fish in a variety of habitats. Habitats included natural brush piles, logs, root masses, undercut banks, rocks, tires, shopping carts, and any other structures that could provide cover. Stunned fishes were collected in the nets and placed into buckets for processing. Once the entire reach had been shocked, the collection team returned to the beginning of the reach to process the collection. 


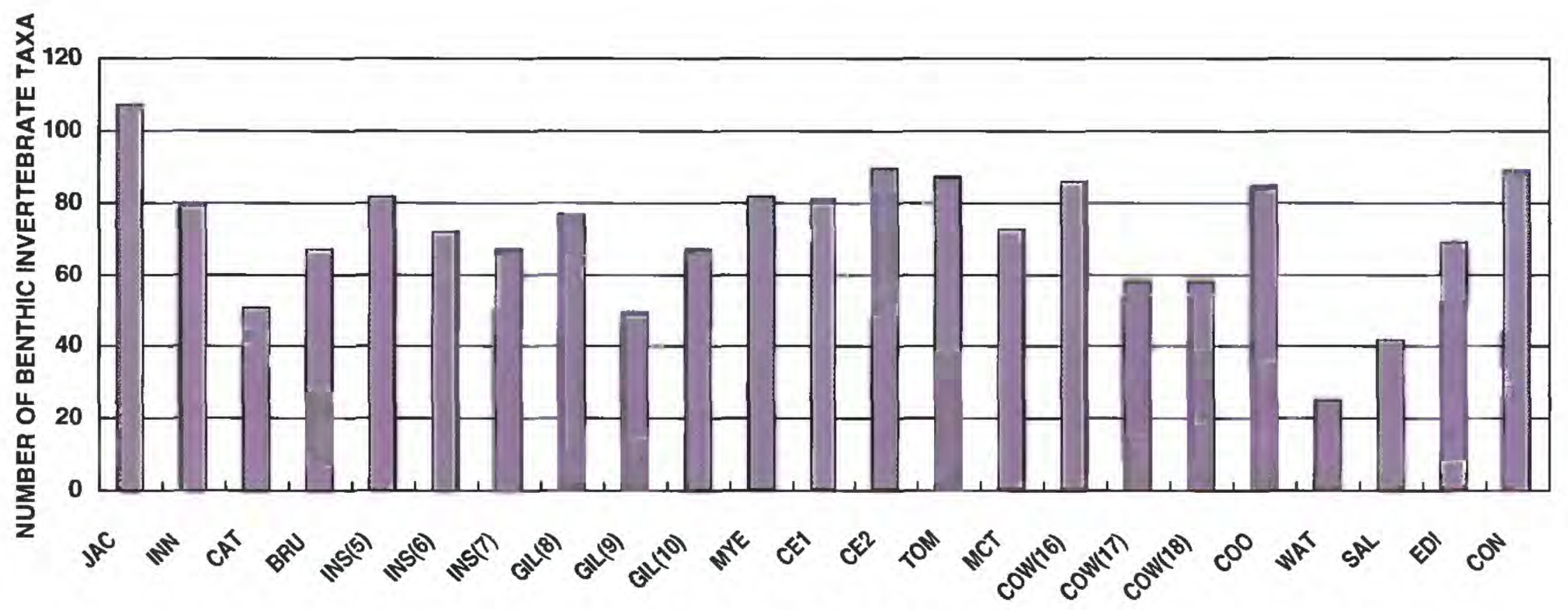

\section{STREAM REACH}

Figure 4. Number of benthic invertebrate taxa identified in each stream reach of the Santee River Basin and Coastal Drainages study unit, North and South Carolina, 1996-98.

[JAC, Jacob Fork near Ramsey, N.C., USGS 02143040 (reach 1); INN, Indian Creek near Laboratory, N.C., USGS 21435000 (reach 2); CAT, South Fork Catawba River at McAdenville, N.C, USGS 02145112 (reach 3); BRU, Brushy Creek near Pelham, S.C., USGS 021603257 (reach 4); INS(5), INS(6), INS(7), Indian Creek above Newberry, S.C., USGS 021607224 (reaches 5, 6, and 7); GIL(8), Gills Creek near Hopkins, S.C., USGS 02169595 (reach 8); GIL(9) and GIL(10), Gills Creek at Columbia, S.C., USGS 02169570 (reaches 9 and 10); MYE, Myers Creek near Hopkins. S.C., USGS 02169660 (reach 11); CE1, Cedar Creek below Myers Creek near Hopkins, S.C., USGS 02169670 (reach 12); CE2. Cedar Creek at Cedar Creek Hunt Club near Gadsden, S.C., USGS 02169672 (reach 13); TOM. Toms Creek near Gadsden. S.C., USGS 021696966 (reach 14); MCT, McTier Creek near Monetta, S.C., USGS 02172300 (reach 15): COW(16), Cow Castle Creek near Bowman, S.C., USGS 02174250 (reach 16); COW(17), Cow Castle Creek near Bowman. S.C., USGS 02174250 (reach 17); COW(18), Cow Castle Creek near Bowman, S.C., USGS 02174250 (reach 18); COO, Coosawhatchie River near Grays, S.C., 02176518 (reach 19); WAT, Wateree River near Camden, S.C., USGS 02148000 (reach 20); SAL, Saluda River near Columbia, S.C., USGS 02169000 (reach 21); EDI, Edisto River near Cottageville, SC, USGS 02174175 (reach 22); CON, Congaree River at Columbia, S.C., USGS 02169500 (reach 23)]

Fish collection by barge or boat was conducted in a downstream direction, with the current, so that shocked fish could more easily be netted as they floated downstream. Two passes were made along each bank of the stream or river. The fishes were held in a live-well or large bucket in preparation for processing.

The captured fishes were identified, weighed, measured (standard length and total length), and observed for anomalies such as lesions. tumors, parasites, and frayed fins. Afterwards, they were placed in a holding reservoir to prevent them from being recaptured.

The "shock and catch" process was performed a second time. If additional species were collected, a third pass was performed. All fishes were released when the processing was completed; more than 90 percent of the fishes survived the processing and were returned to the stream. Two or three individuals of each species identified were preserved in 10 percent buffered formaldehyde as voucher specimens. Fishes that could not be readily identified onsite were preserved and taken to the laboratory for identification. The primary taxonomic authority for the field identifications was from Rohde and others (1994). Identification was verified by Fritz Rohde, North Carolina Department of Marine Fisheries in Wilmington, under contract to the USGS. The number and species of fishes collected at each site in the study unit are shown in figure 5 and table 31 , respectively. 


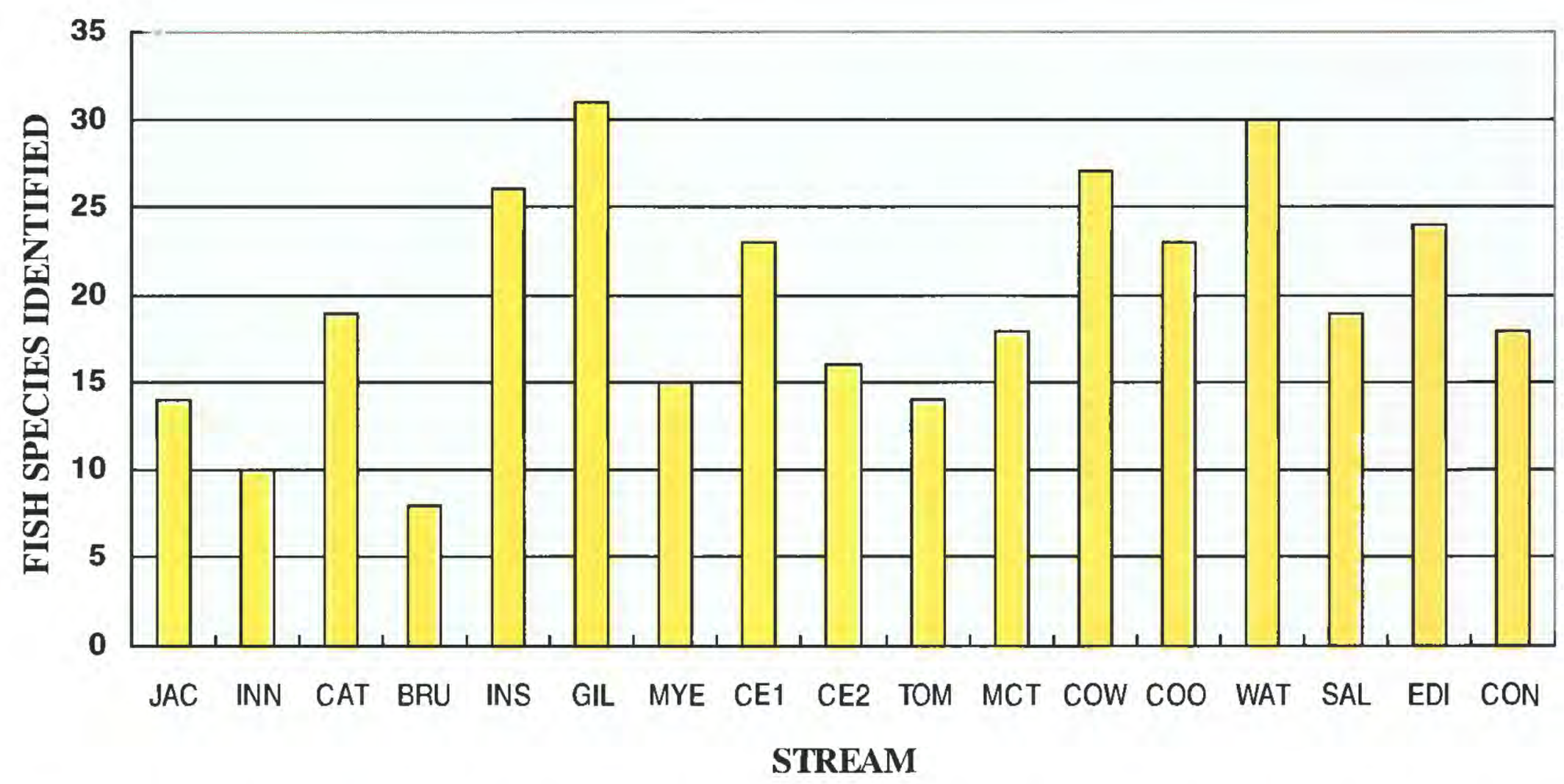

Figure 5. Number of fish species identified in each stream of the Santee River Basin and Coastal Drainages study unit, North and South Carolina, 1996-98.

[JAC, Jacob Fork near Ramsey, N.C., USGS 02143040 (reach 1); INN, Indian Creek near Laboratory, N.C., USGS 21435000 (reach 2); CAT, South Fork Catawba River at McAdenville, N.C, USGS 02145112 (reach 3); BRU, Brushy Creek near Pelham, S.C., USGS 021603257 (reach 4); INS, Indian Creek above Newberry, S.C., USGS 021607224 (reaches 5, 6, and 7); GIL, Gills Creek near Hopkins, S.C., USGS 02169595 (reach 8 ) and Gills Creek at Columbia, S.C., USGS 02169570 (reaches 9 and 10); MYE, Myers Creek near Hopkins, S.C., USGS 02169660 (reach 11); CE1, Cedar Creek below Myers Creek near Hopkins, S.C., USGS 02169670 (reach 12); CE2, Cedar Creek at Cedar Creek Hunt Club near Gadsden, S.C., USGS 02169672 (reach 13); TOM, Toms Creek near Gadsden, S.C., USGS 021696966 (reach 14); MCT. McTier Creek near Monetta. S.C.. USGS 02172300 (reach 15); COW, Cow Castle Creek near Bowman, S.C., USGS 02174250 (reaches 16, 17, and 18); COO, Coosawhatchie River near Grays, S.C., 02176518 (reach 19); WAT, Wateree River near Camden, S.C., USGS 02148000 (reach 20); SAL, Saluda River near Columbia, S.C., USGS 02169000 (reach 21); EDI, Edisto River near Cottageville, S.C., USGS 02174175 (reach 22); CON, Congaree River at Columbia, S.C., USGS 02169500 (reach 23)]

\section{SUMMARY}

An evaluation of several ecological components of 16 streams was made during 1996-98 as part of the U.S. Geological Survey National Water Quality Assessment Program in the Santee River Basin and Coastal Drainages study unit. Data were collected to evaluate habitat structure, aquatic community structure and composition, and water quality. These data are available for use with physical and chemical water-quality data, to relate streamwater quality and aquatic-community health to human land-use activities. This report presents the ecological data in tabular form.

\section{SELECTED REFERENCES}

Abrahamsen, T.A., 1999, Trace elements in bed sediments and biota from streams in the Santee River Basin and Coastal Drainages, North and South Carolina, 1995-97: U.S. Geological Survey Water-Resources Investigations Report 99- 4179, 26 p.

Abrahamsen, T.A., Hughes, W.B., Reuber, E.J., and Sicherman, T.L., 1997, Selected aquatic ecology, surface-water quality, and ground-water studies in the Santee River Basin and Coastal Drainages, North and South Carolina: Columbia, U.S. Geological Survey, Open-File Report 97-115, 49 p.

Biggs, B.J.F., Duncan, M.J., Jowett, I.G., Quinn, J.M., Hickey, C.W., Davies-Colley, R.J., and Close, M.E., 1990, Ecological characterisation, classification, and 
modeling of New Zealand rivers: An introduction and synthesis: New Zealand Journal of Marine and Freshwater Research, v. 24, p. 277-304.

Cuffney, T.F., Gurtz, M.E., and Meador, M.R., 1993, Methods for collecting benthic invertebrate samples as part of the National Water-Quality Assessment Program: U.S. Geological Survey Open-File Report 93-406, $66 \mathrm{p}$.

Fitzpatrick, F.A., Waite, I.R., D’Arconte, P.J., Meador, M.R., Maupin, M.A., and Gurtz, M.E., 1998, Revised methods for characterizing stream habitat in the National Water-Quality Assessment Program: U.S. Geological Survey Water- Resources Investigations Report 98-4052.67 p.

Hawkins, C.P., 1985, Substrate associations and longitudinal distributions in species of Ephemerellidae

(Ephemeroptera: Insecta) from western Oregon: Freshwater Invertebrate Biology, v. 4, p. 181-188.

Hughes, W.B., Abrahamsen, T.A., Maluk, T.L., Reuber, E.J.. and Wilhelm, L.J., 2000, Water quality in the Santee River Basin and Coastal Drainages, North and South Carolina, 1995-98, U.S. Geological Survey Circular 1206, 32 p.

Hupp, C.R., 1986, Upstream variation in bottomland vegetation patterns, northwestern Virginia: Bulletin of the Torrey Botanical Club, v. 113, p. 421-430.

Leahy, P.P., Rosenshein, J.S., and Knopman, D.S., 1990, Implementation plan for the National Water-Quality Assessment Program:, U.S. Geological Survey, OpenFile Report 90-174, 10 p.

Leopold, L.B., Wolman, M.G., and Miller, J.P., 1964, Fluvial processes in geomorphology: San Francisco, W.H. Freeman, $522 \mathrm{p}$.

Maluk, T.L., 2000, Spatial and seasonal variability of nutrients, pesticides, bacteria, and suspended sediment in the Santee River Basin and Coastal Drainages, North and South Carolina, 1995-97: U.S. Geological Survey Water-Resources Investigations Report 00-4076, 46 p.
Meador, M.R., Cuffney, T.F., and Gurtz, M.E., 1993a, Methods for sampling fish communities as a part of the National Water-Quality Assessment Program: U.S. Geological Survey Open-File Report 93-104, 40 p.

Meador, M.R., Hupp, C.L., Cuffney, T.F., and Gurtz, M.E., 1993b, Methods for characterizing stream habitat as part of the National Water-Quality Assessment Program: U.S. Geological Survey, Open-File Report 93-408, 48 p.

Mueller-Dombois, Dieter, and Ellenberg, Heinz, 1974, Aims and methods of vegetation ecology: New York, John Wiley, $547 \mathrm{p}$.

Omernik, J.M., 1987, Ecoregions of the conterminous United States: Annals of the Association of American Geographers, v. 77, no. 1, p. $118-125$.

1995, Ecoregions: A spatial framework for environmental management, in Davis, W.S., and Thorpe, P.S., eds.. Biological assessment and criteria: Tools for water resource planning and decision-making: Boca Raton, Fla., Lewis Publishers, 415 p.

Plafkin, J.L., Barbour, M.T., Porter. K.D.. Gross, S.K., and Hughes, R.M., 1989, Rapid bioassessment protocols for use in streams and rivers: U.S. Environmental Protection Agency, variously paged.

Platts, W.S., Megahan, W.F., and Minshall, G.W., 1983, Methods for evaluating stream, riparian, and biotic conditions: Ogden, Utah, U.S. Forest Service General Technical Report No. INT-138, 70 p.

Porter, S.D., Cuffney, T.F., Gurtz, M.E., and Meador, M.R., 1993, Methods for collecting algal samples as part of the National Water Quality Assessment Program: U.S. Geological Survey Open-File Report 93-409, 39 p.

Rohde, F.C., Arndt, R.G., Lindquist, D.G., and Parnell, J.F., 1994, Freshwater fishes of the Carolinas, Virginia, Maryland, and Delaware: Chapel Hill, University of North Carolina Press, 222 p. 


\section{Habitat Data}




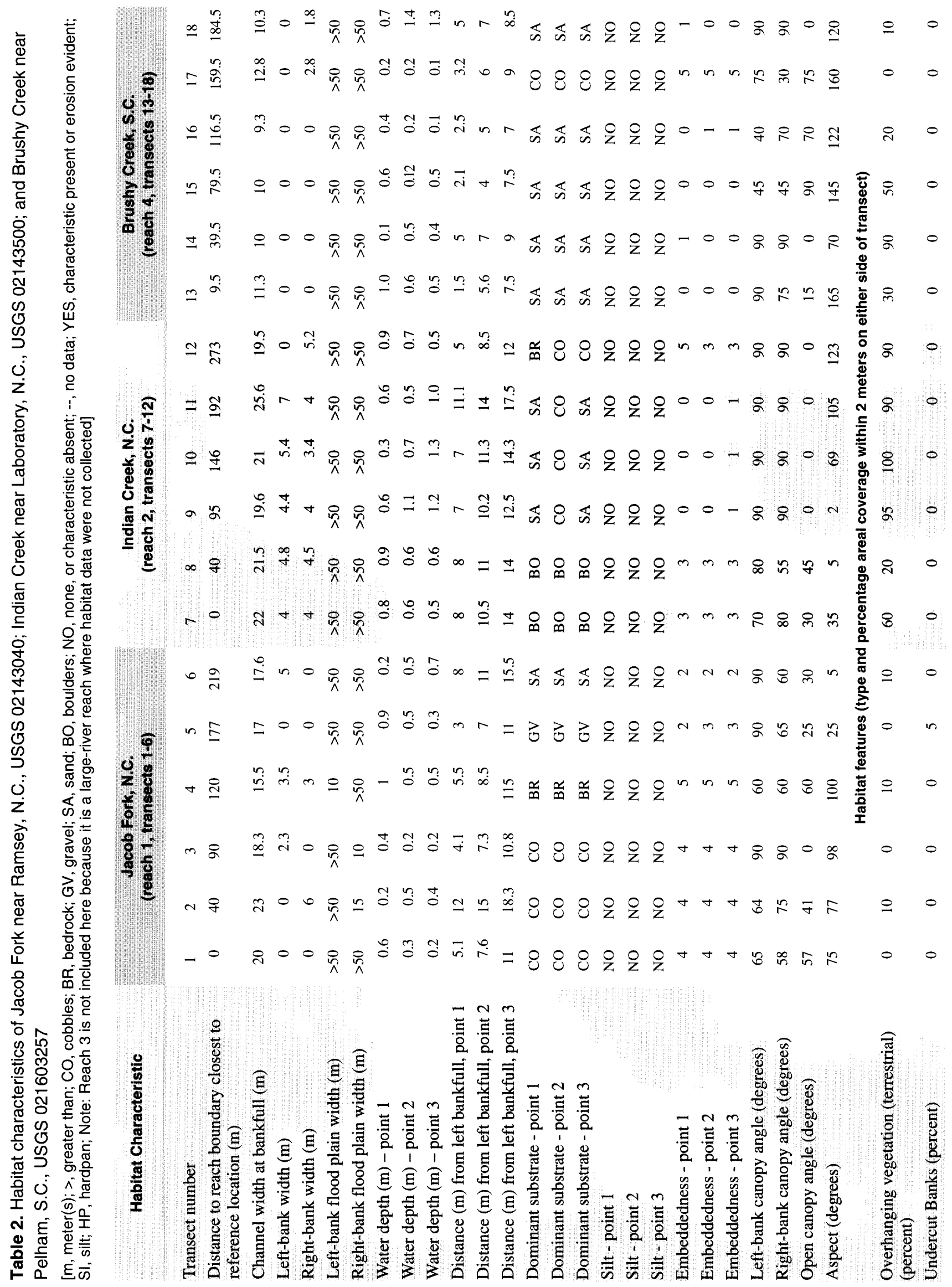




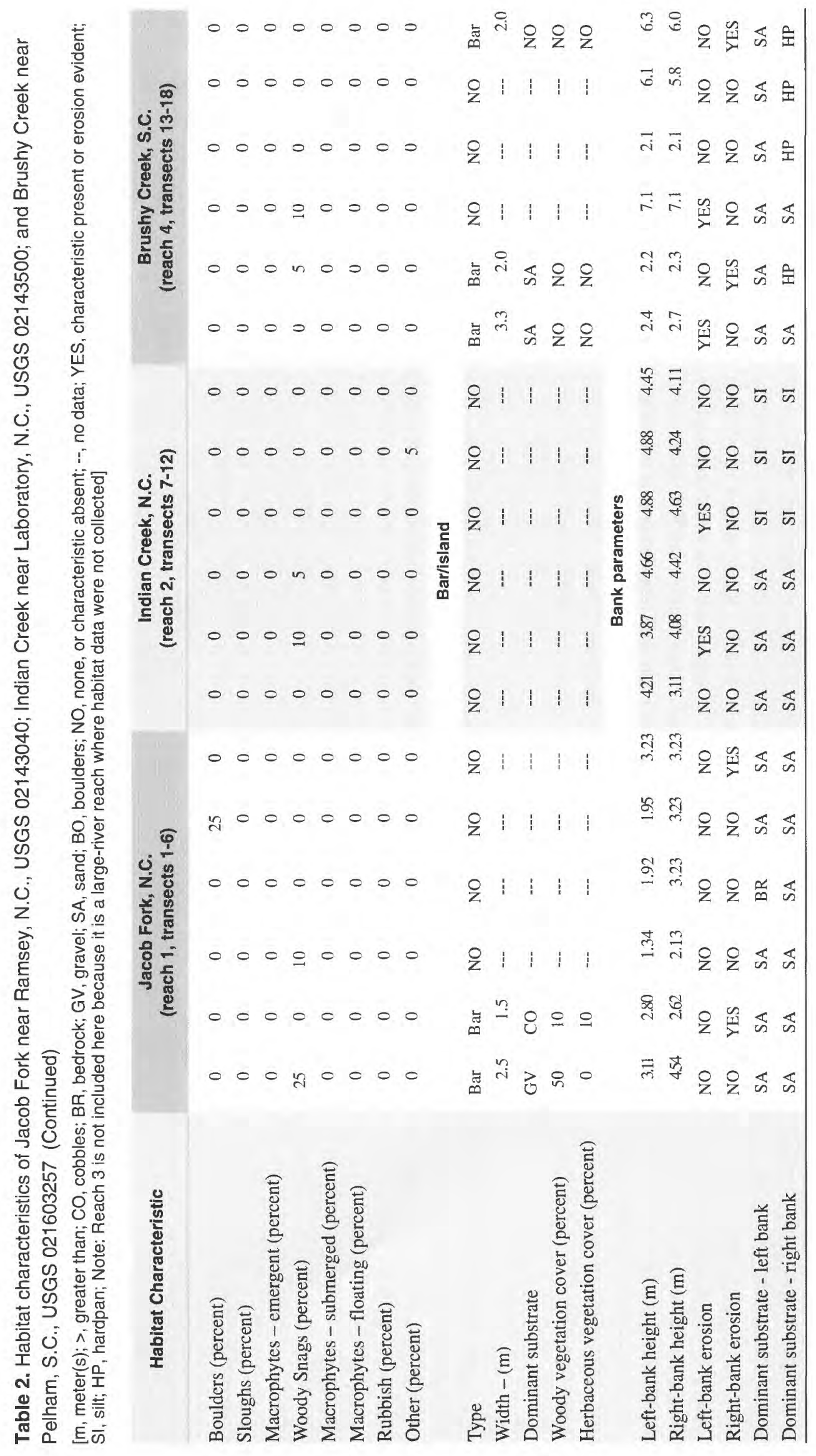




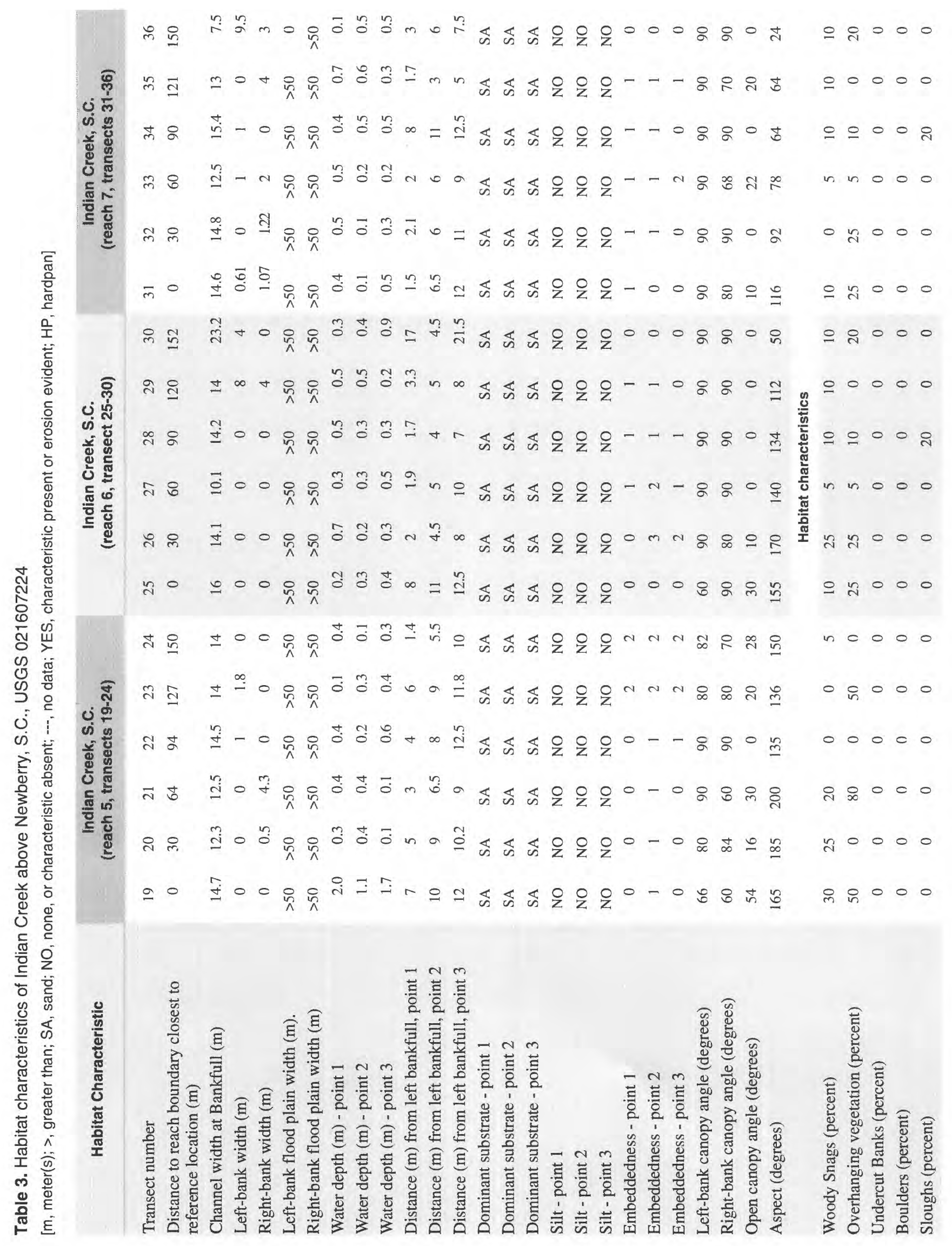




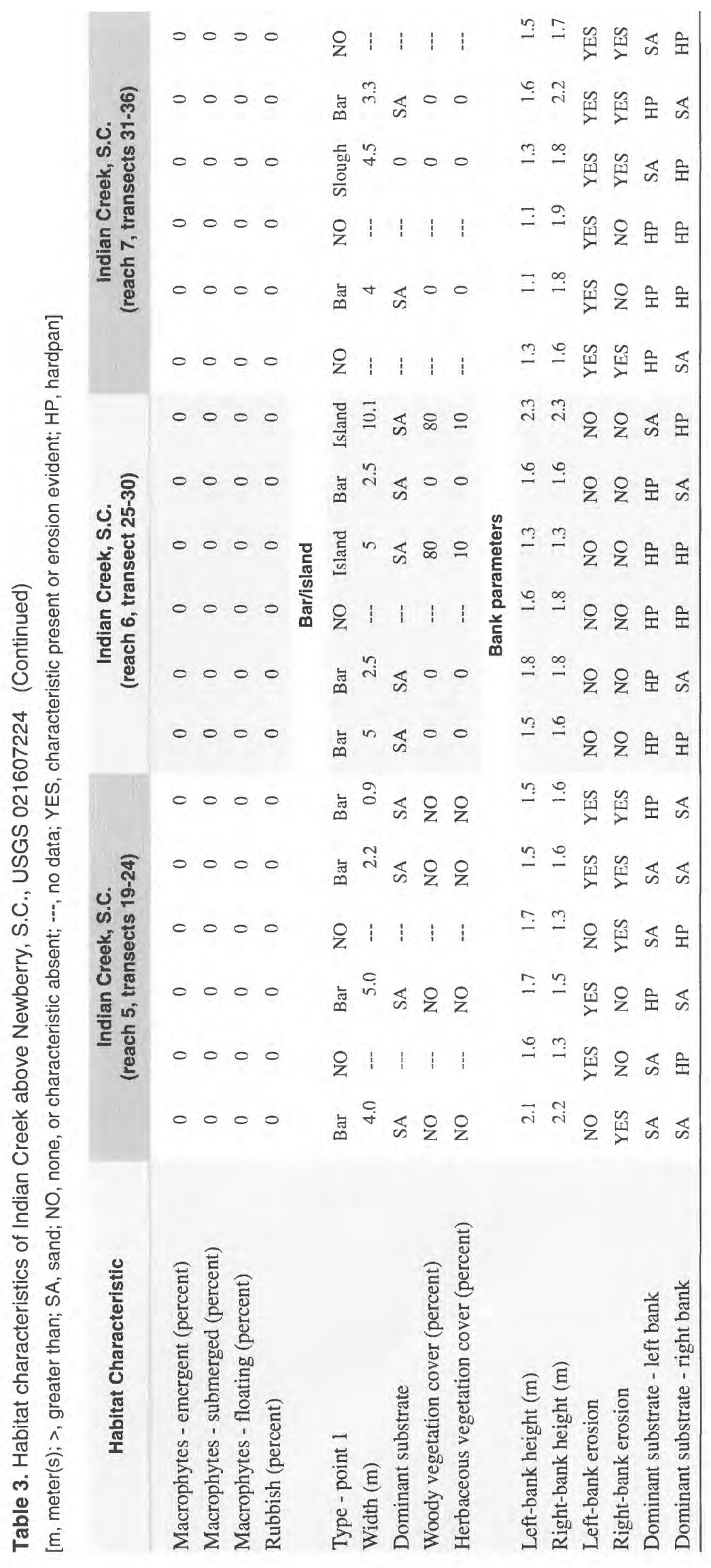




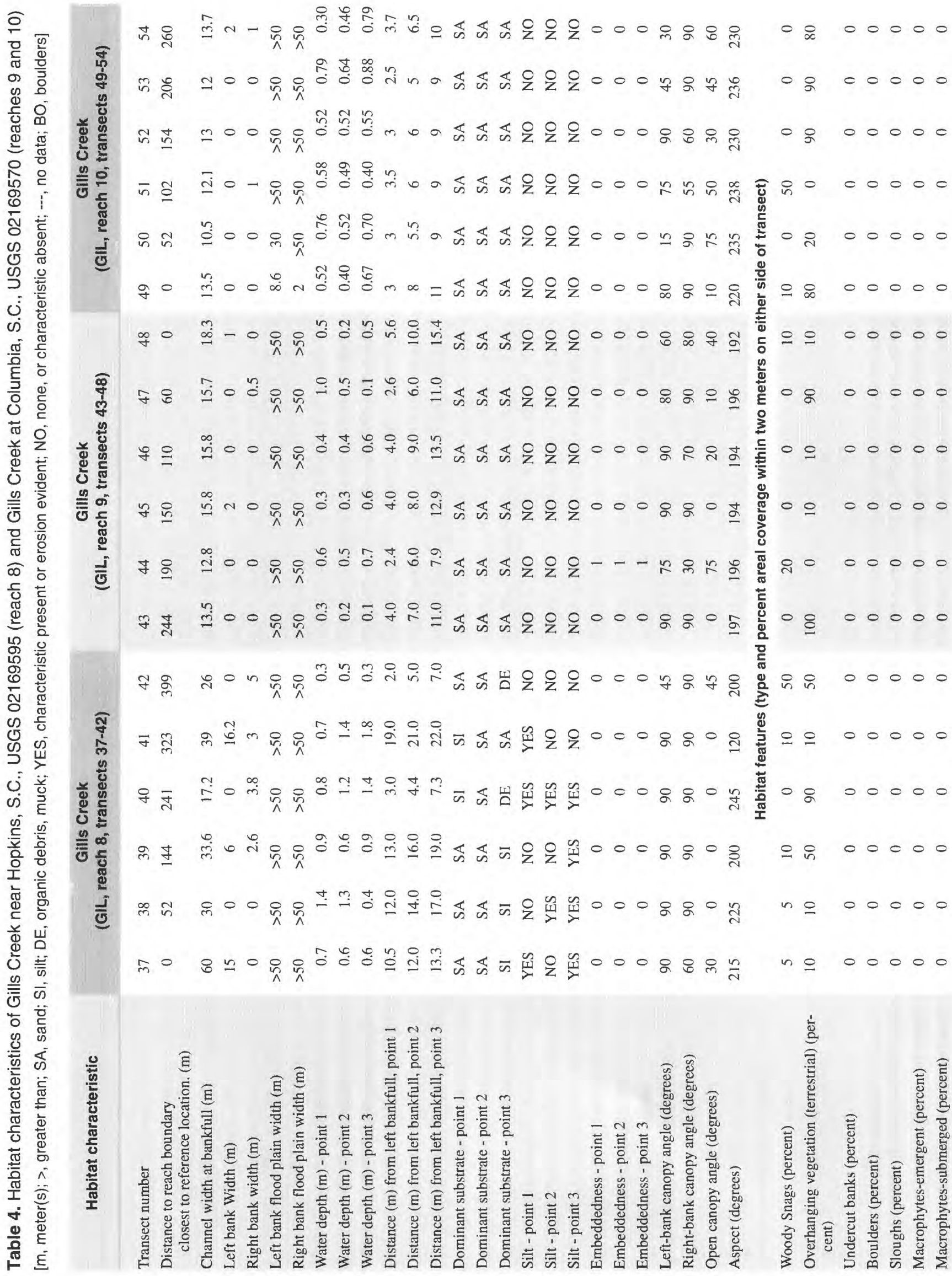




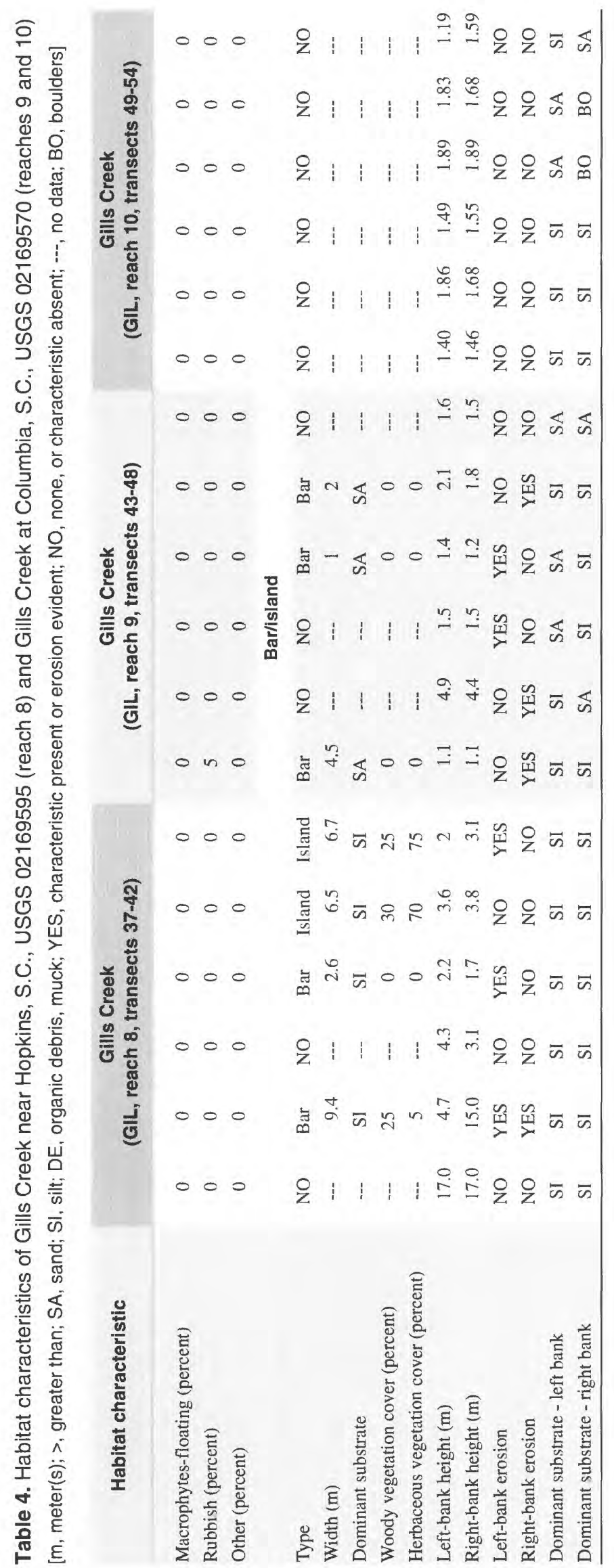




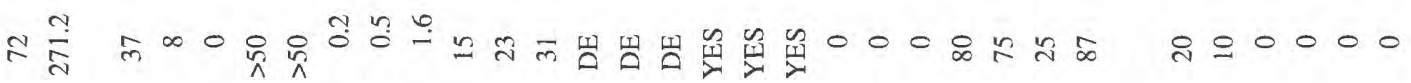

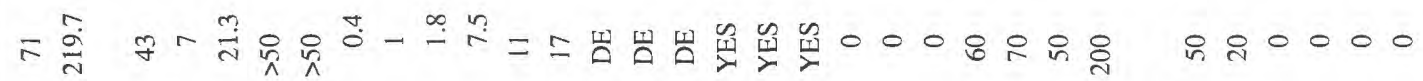

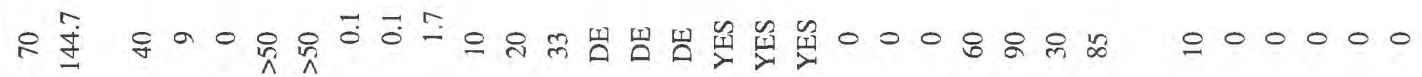

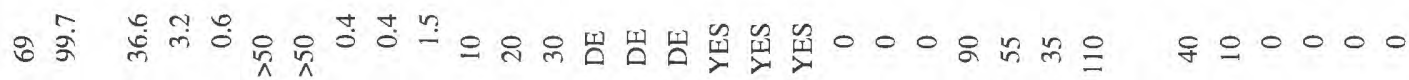

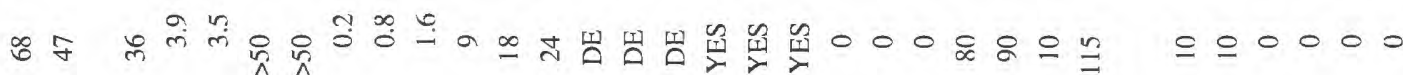

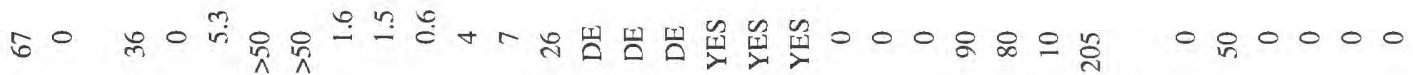

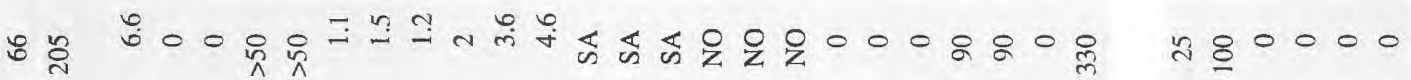

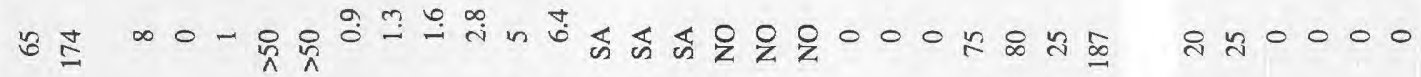

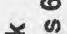

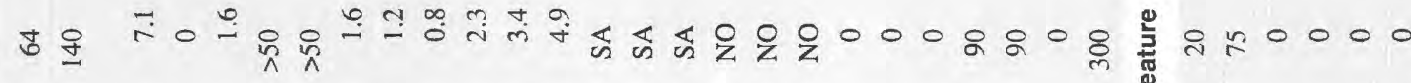
 잉

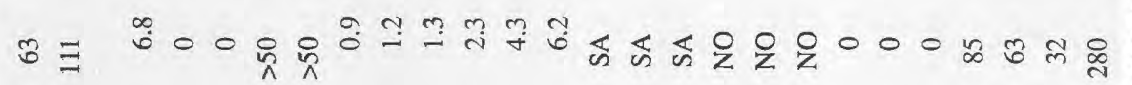

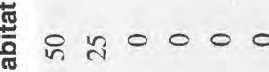
密 ㅇำ

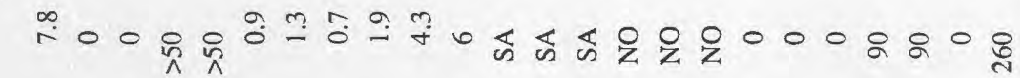

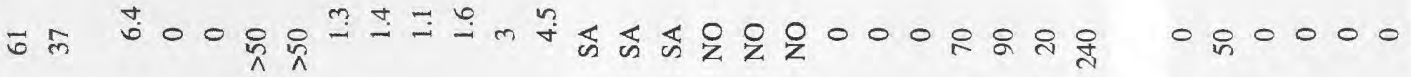

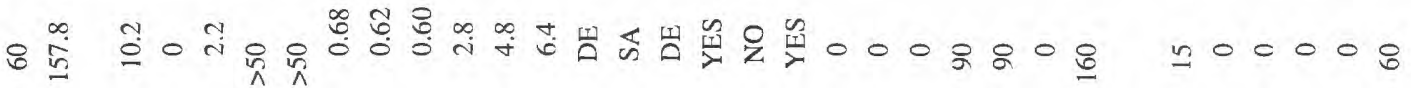

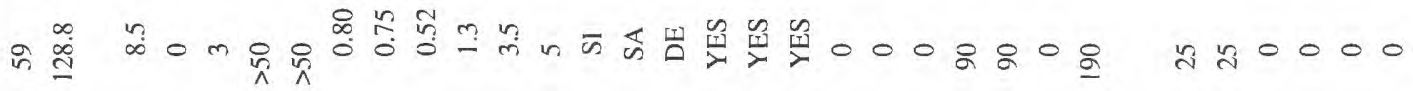

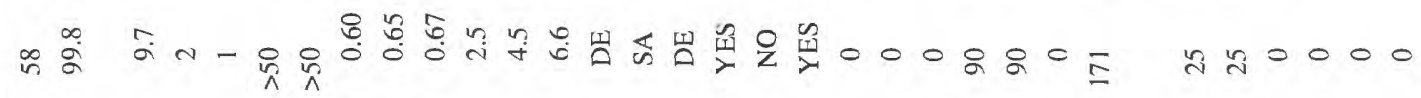

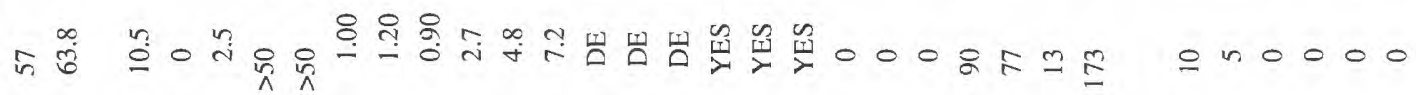

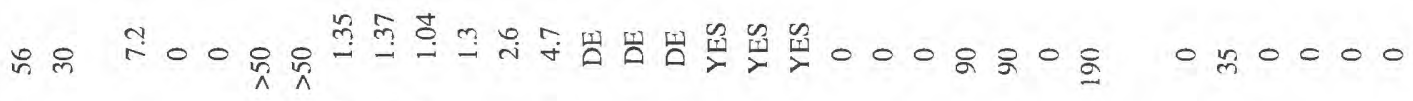

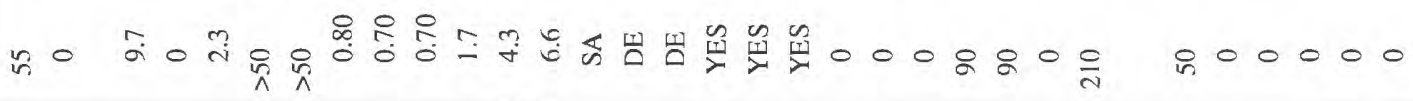




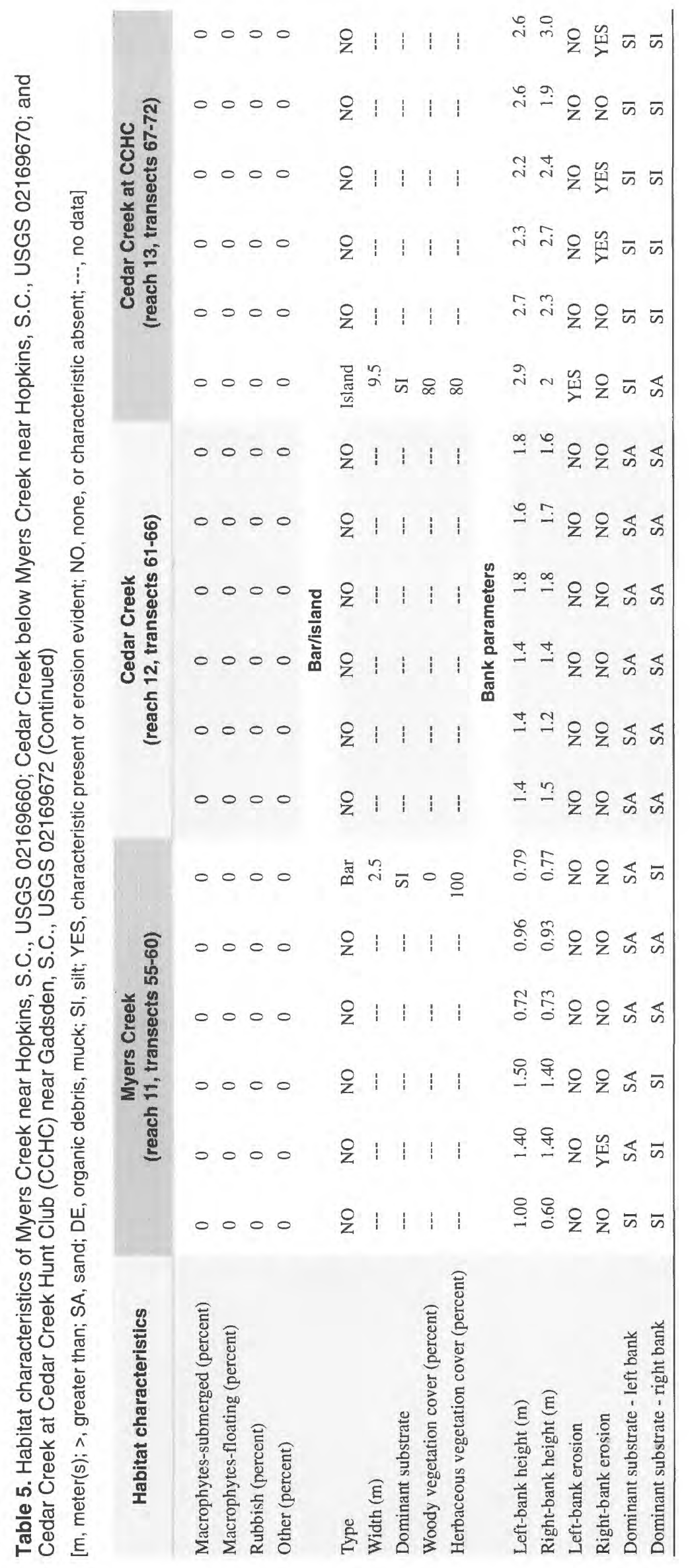




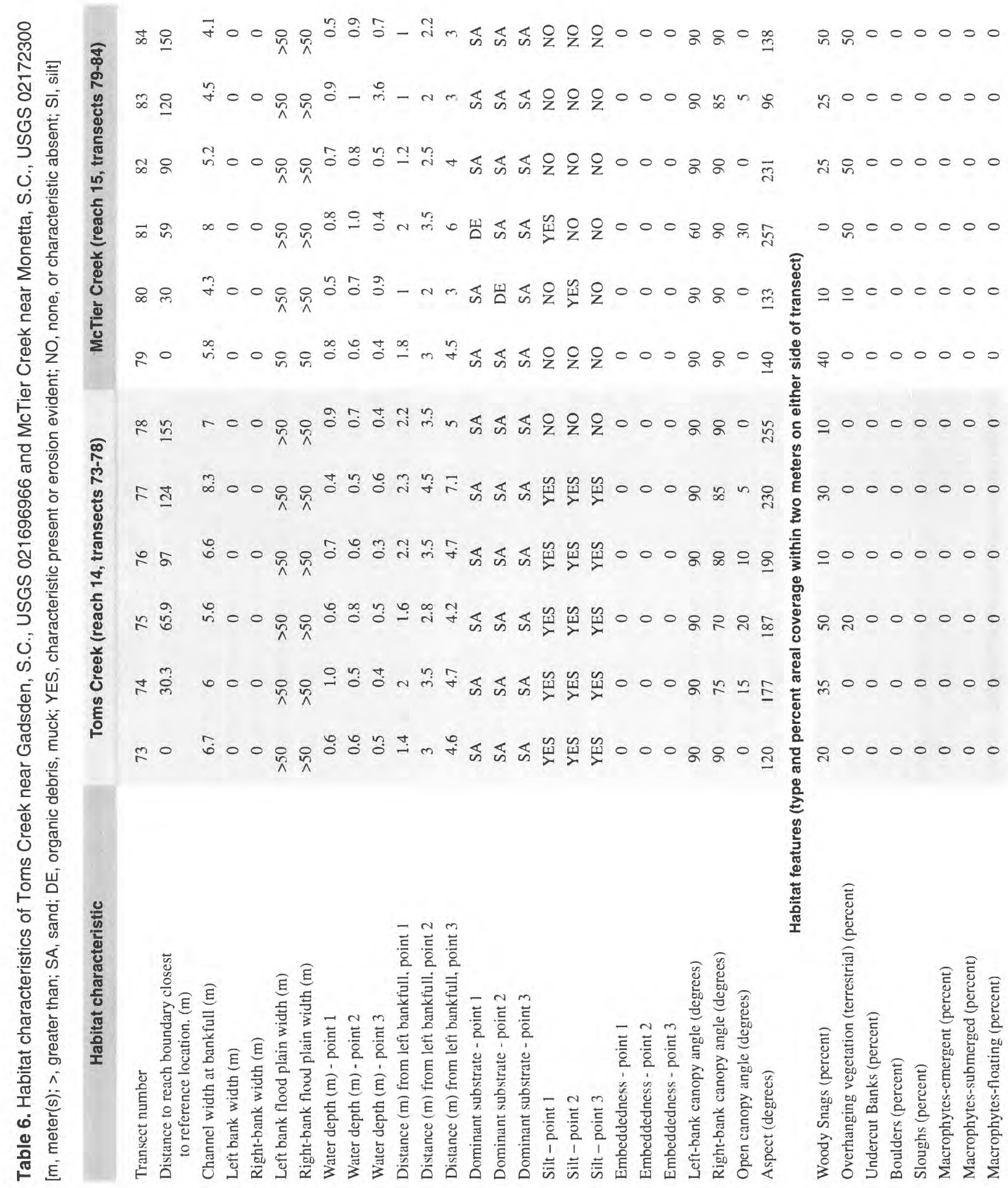




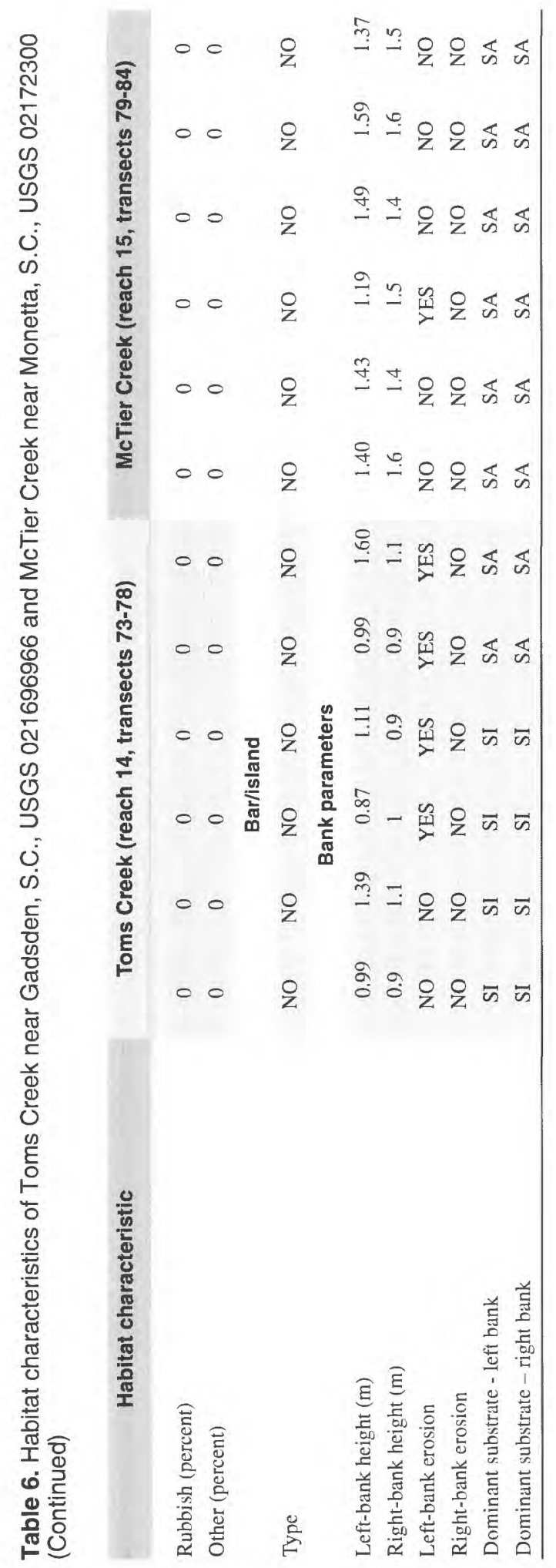




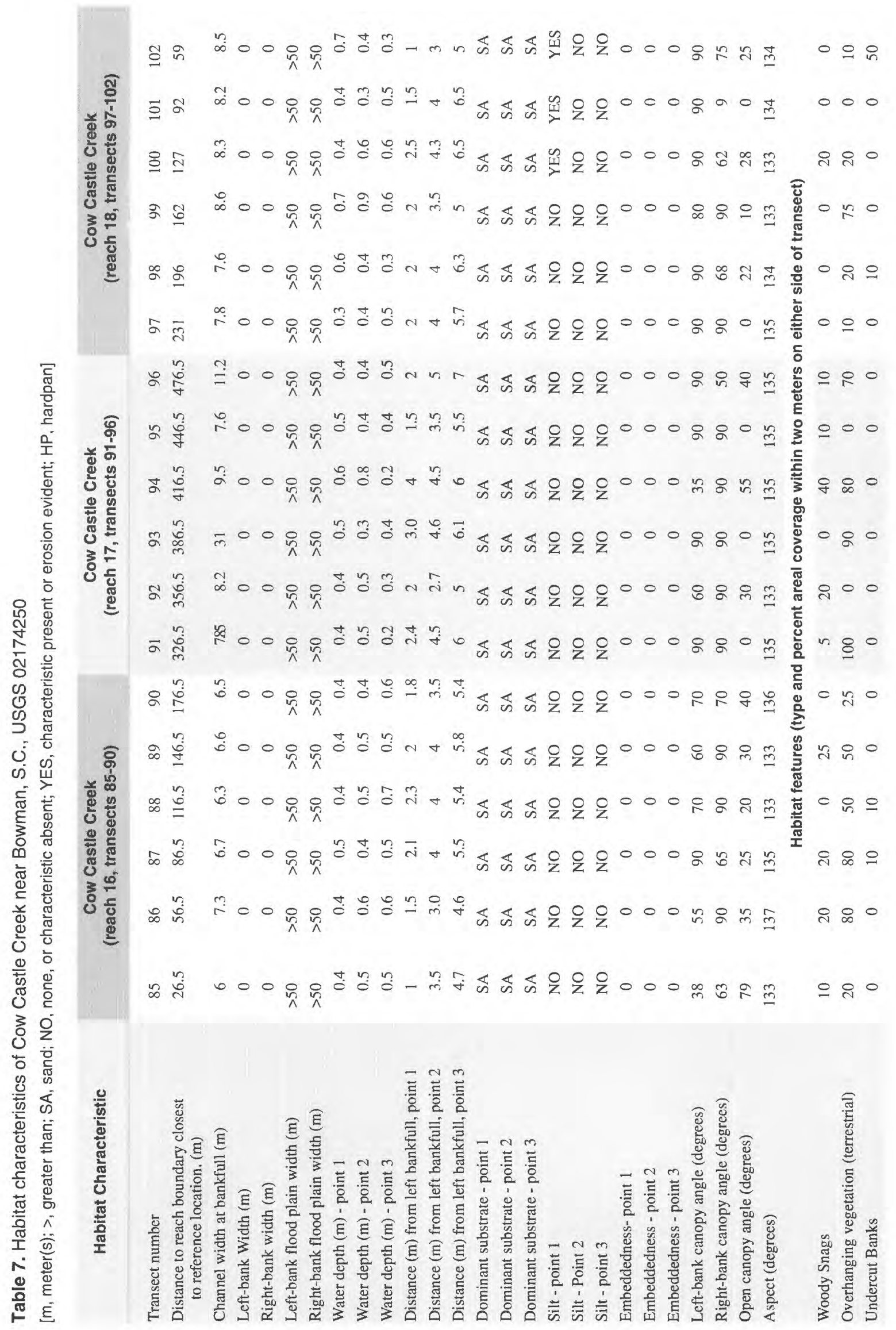




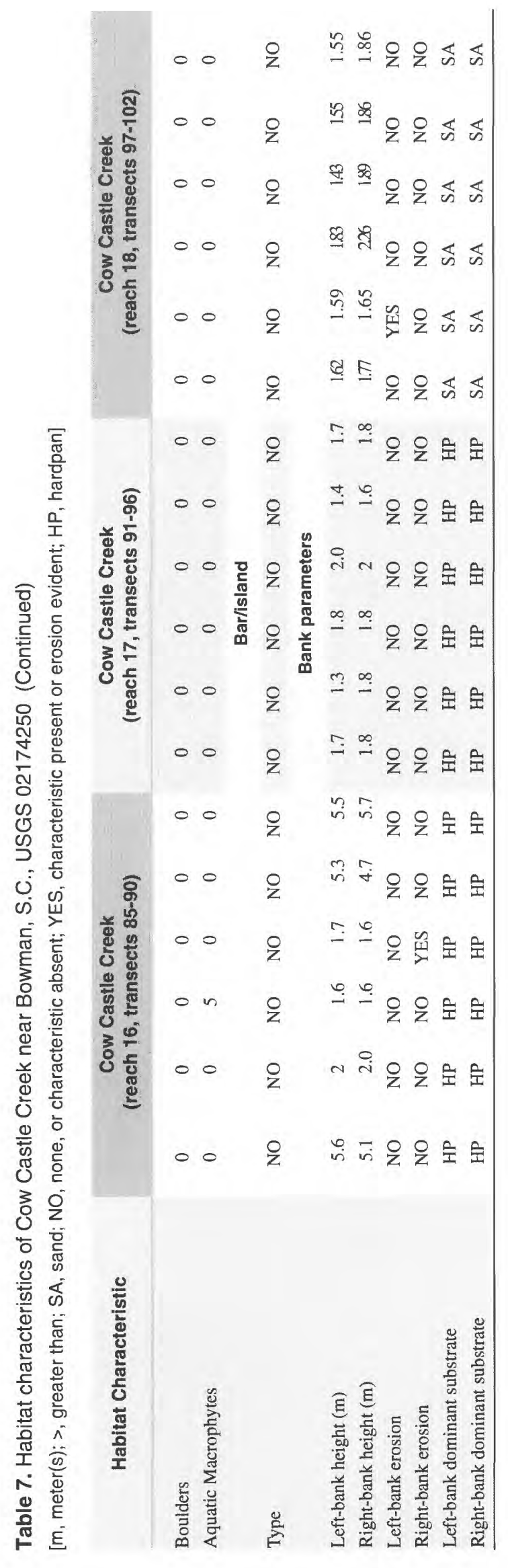


Table 8. Habitat characteristics of the Coosawhatchie River near Grays, S.C., USGS 02176518 [m, meter(s); >, greater than; DE, organic debris, muck; SA, sand; SI, silt; NO, none, or characteristic absent; YES, characteristic present or erosion evident]

\section{Habitat Characteristic}

Transect number

Distance to reach boundary closest to reference location. (m)

Channel width at bankfull $(\mathrm{m})$

Left-bank Width (m)

Right-bank width (m)

Lett-bank tinod plain width (m)

Right-bank flood plain width (m)

Water depth (m) - point 1

Water depth (m) - point 2

Water depth $(\mathrm{m})$ - point 3

Distance (m) trom lett banktull. point 1

Distance $(\mathrm{m})$ from left bankfull, point 2

Distance $(m)$ from left bankfull, point 3

Dominant substrate - point 1

Dominant substrate - point 2

Dominant substrate - point 3

Embeddedness - point 1

Embeddedness - point 2

Embeddedness - point 3

Silt - point I

Silt - point 2

Silt - point 3

Lett-bank canopy angle (degrees)

Right-bank canopy angle (degrees)

Open canopy angle (degrees)

Aspect (degrees)

Woody Snags (percent)

Overhanging vegetation (terrestrial) (percent)

Undercut Banks (percent)

Boulders (percent)

Sloughs (percent)

Macrophytes-emergent (percent)

Macrophytes-submerged (percent)

Macrophytes-tloatıng (percent)

Rubbish (percent)

Other (percent)

Left-bank height ( $\mathrm{m}$ )

Right-bank height ( $\mathrm{m}$ )

Lett-bank erosion

Right-bank erosion

Domınant substrate - lett bank

Dominant substrate - right bank

\section{Coosawhatchie River (reach 19, transects 103-108)}

\begin{tabular}{|c|c|c|c|c|}
\hline 103 & 104 & 105 & 106 & 107 \\
\hline 0 & 60 & 110 & 193 & 251 \\
\hline 35 & 26.8 & 49.6 & 22 & 26 \\
\hline 6.5 & 7 & 6 & 3.4 & 1.6 \\
\hline 2.2 & 1.7 & 19.8 & 1 & 5 \\
\hline$>50$ & $>50$ & $>50$ & $>50$ & $>50$ \\
\hline$>50$ & $>50$ & $>50$ & $>50$ & $>50$ \\
\hline 3.1 & 2.1 & 0.7 & 1.0 & 0.9 \\
\hline 4.2 & 1.7 & 0.9 & 1.6 & 1 \\
\hline 1.8 & 1.2 & 1.1 & 1.1 & 1.6 \\
\hline 12.1 & 13 & 3 & 7.4 & 5 \\
\hline 18 & 18 & 15 & 11.4 & 10 \\
\hline 24 & 23 & 30 & 15 & 15 \\
\hline DE & $\mathrm{SA}$ & $\mathrm{DE}$ & $\mathrm{SA}$ & DE \\
\hline SA & SA & SA & SA & DE \\
\hline $\mathrm{DE}$ & $\mathrm{DE}$ & $\mathrm{DE}$ & SA & $\mathrm{DE}$ \\
\hline 13 & $u$ & (1) & 11 & 0 \\
\hline 0 & 0 & 0 & 0 & 0 \\
\hline 0 & 0 & 0 & 0 & 0 \\
\hline NU & NU & NO & $\mathrm{NU}$ & NU \\
\hline NO & NO & NO & NO & NO \\
\hline NO & NO & NO & NO & NO \\
\hline y(1) & 90 & 90 & yu & 90 \\
\hline 90 & 90 & 90 & 90 & 90 \\
\hline 0 & 0 & 0 & 0 & 0 \\
\hline 175 & 260 & 0 & 220 & 135 \\
\hline
\end{tabular}

Habitat features

\begin{tabular}{|c|c|c|c|c|c|}
\hline 20 & 0 & 0 & 0 & 0 & 0 \\
\hline 100 & 0 & 0 & 0 & 0 & 0 \\
\hline U & 0 & 0 & 0 & 0 & 0 \\
\hline U & 0 & 0 & 0 & 0 & 0 \\
\hline 0 & 0 & 0 & 0 & 0 & 0 \\
\hline 0 & 0 & 0 & 0 & 0 & 0 \\
\hline 0 & 0 & 0 & 0 & 0 & 0 \\
\hline 0 & 0 & 0 & 0 & 0 & 0 \\
\hline 0 & 0 & 0 & 0 & 0 & 0 \\
\hline 0 & 0 & 0 & 0 & 0 & 0 \\
\hline \multicolumn{6}{|c|}{ Bar/island } \\
\hline NO & NO & $\mathrm{NO}$ & NO & $\mathrm{NO}$ & $\mathrm{NO}$ \\
\hline \multicolumn{6}{|c|}{ Bank parameters } \\
\hline 2.04 & 2.33 & 2.33 & 1.86 & 2.59 & 1.28 \\
\hline 2.04 & 2.33 & 2.33 & 1.86 & 2.90 & 1.68 \\
\hline NU & NU & NU & NU & NU & $\mathrm{NU}$ \\
\hline $\mathrm{NO}$ & $\mathrm{NO}$ & $\mathrm{NO}$ & $\mathrm{NO}$ & NO & $\mathrm{NO}$ \\
\hline DE & $\mathrm{DE}$ & $\mathrm{DE}$ & $D E$ & $\mathrm{DE}$ & DE \\
\hline $\mathrm{DE}$ & $\mathrm{DE}$ & $\mathrm{DE}$ & $\mathrm{DE}$ & $\mathrm{DE}$ & $\mathrm{DE}$ \\
\hline
\end{tabular}


Riparian-Zone Data 
Table 9. Riparian-zone trees, Jacob Fork near Ramsey, N.C., USGS site 02143040 (reach 1)

[m, meter(s); T-n, transect number; LQPn, left quarterpoint, nth tree; RQPn, right quarterpoint, $n$th tree]

\begin{tabular}{|c|c|c|c|c|c|}
\hline $\begin{array}{c}\text { Tree } \\
\text { position }\end{array}$ & Species code & Scientific name & Common name & $\begin{array}{c}\text { Distance } \\
\text { from pivot } \\
\text { point }(\mathrm{m})\end{array}$ & $\begin{array}{l}\text { Trunk diameter at } \\
\text { breast height (m) }\end{array}$ \\
\hline T-1 LQP1 & BENI & Betula nigra & river birch & 1.3 & 0.50 \\
\hline T-1 LQP2 & PIST & Pinus stroba & white pine & 2.1 & 0.02 \\
\hline T-1 LQP3 & CACA & Carpinus caroliniana & ironwood & 1.5 & 0.04 \\
\hline T-1 LQP4 & CACA & Carpinus caroliniana & ironwood & 1.3 & 0.11 \\
\hline T-1 RQP1 & CACA & Carpinus caroliniana & ironwood & 0.5 & 0.06 \\
\hline T-1 RQP2 & RHMA & Rhododendron maximum & great rhododendron & 0.6 & 0.06 \\
\hline T-1 RQP3 & RHMA & Rhododendron maximum & great rhododendron & 0.9 & 0.04 \\
\hline T-1 RQP4 & RHMA & Rhododendron maximum & great rhododendron & 3.6 & 0.07 \\
\hline T-2 LQP1 & ILOP & Ilex opaca & American holly & 4.5 & 0.03 \\
\hline T-2 LQP2 & ACPE & Acer pensylvanicum & striped maple & 1.5 & 0.02 \\
\hline T-2 LQP3 & CACA & Carpinus caroliniana & ironwood & 3.2 & 0.09 \\
\hline T-2 LQP4 & CACA & Carpinus caroliniana & ironwood & 3.3 & 0.17 \\
\hline T-2 RQP1 & CACA & Carpinus caroliniana & ironwood & 2.3 & 0.15 \\
\hline T-2 RQP2 & $\mathrm{CACA}$ & Carpinus caroliniana & ironwood & 2.0 & 0.08 \\
\hline T-2 RQP3 & QUAL & Quercus alba & white oak & 2.5 & 0.57 \\
\hline T-2 RQP4 & RHMA & Rhododendron maximum & great rhododendron & 0.9 & 0.02 \\
\hline T-3 LQP1 & CACA & Carpinus caroliniana & ironwood & 1.7 & 0.10 \\
\hline T-3 LQP2 & CACA & Carpinus caroliniana & ironwood & 2.0 & 0.06 \\
\hline T-3 LQP3 & BENI & Betula nigra & river birch & 2.1 & 0.36 \\
\hline T-3 LQP4 & CACA & Carpinus caroliniana & ironwood & 5.1 & 0.12 \\
\hline T-3 RQP1 & COFL & Cornus florida & flowering dogwood & 3.3 & 0.07 \\
\hline T-3 RQP2 & ACRU & Acer rubrum & red maple & 4.9 & 0.02 \\
\hline T-3 RQP3 & CACA & Carpinus caroliniana & ironwood & 1.8 & 0.09 \\
\hline T-3 RQP4 & CACA & Carpinus caroliniana & ironwood & 3.7 & 0.18 \\
\hline T-4 LQP1 & ACRU & Acer rubrum & red maple & 1.0 & 0.06 \\
\hline T-4 LQP2 & KALA & Kalmia latifolia & mountain laurel & 0.8 & 0.05 \\
\hline T-4 LQP3 & KALA & Kalmia latifolia & mountain laurel & 1.0 & 0.09 \\
\hline T-4 LQP4 & KALA & Kalmia latifolia & mountain laurel & 1.5 & 0.05 \\
\hline T-4 RQP1 & TSCA & Tsuga canadensis & eastern hemlock & 1.0 & 0.31 \\
\hline T-4 RQP2 & LITU & Liriodendron tulipifera & tulip poplar & 1.5 & 0.19 \\
\hline T-4 RQP3 & RHMA & Rhododendron maximum & great rhododendron & 1.6 & 0.15 \\
\hline T-4 RQP4 & RHMA & Rhododendron maximum & great rhododendron & 2.0 & 0.06 \\
\hline T-5 LQP1 & COFL & Cornus florida & flowering dogwood & 1.9 & 0.11 \\
\hline T-5 LQP2 & ACRU & Acer rubrum & red maple & 2.5 & 0.02 \\
\hline T-5 LQP3 & ILOP & Ilex ораса & American holly & 2.8 & 0.05 \\
\hline T-5 LQP4 & $\mathrm{CACA}$ & Carpinus caroliniana & ironwood & 5.8 & 0.05 \\
\hline T-5 RQP1 & COFL & Cornus florida & flowering dogwood & 1.8 & 0.01 \\
\hline T-5 RQP2 & ACRU & Acer rubrum & red maple & 2.1 & 0.06 \\
\hline T-5 RQP3 & QUAL & Quercus alba & white oak & 2.2 & 0.09 \\
\hline T-5 RQP4 & COFL & Cornus florida & flowering dogwood & 2.9 & 0.03 \\
\hline T-6 LQP1 & KALA & Kalmia latifolia & mountain laurel & 0.5 & 0.03 \\
\hline T-6 LQP2 & KALA & Kalmia latifolia & mountain laurel & 0.8 & 0.04 \\
\hline T-6 LQP3 & KALA & Kalmia latifolia & mountain laurel & 1.2 & 0.04 \\
\hline T-6 LQP4 & KALA & Kalmia latifolia & mountain laurel & 1.3 & 0.03 \\
\hline T-6 RQP1 & ILOP & Ilex opaca & American holly & 1.8 & 0.04 \\
\hline T-6 RQP2 & CACA & Carpinus caroliniana & ironwood & 2.1 & 0.13 \\
\hline T-6 RQP3 & TSCA & Tsuga canadensis & eastern hemlock & 3.9 & 0.24 \\
\hline T-6 RQP4 & CACA & Carpinus caroliniana & ironwood & 3.3 & 0.03 \\
\hline
\end{tabular}


Table 10. Riparian-zone trees, Indian Creek near Laboratory, N.C., USGS 02143500 (reach 2)

[m, meter(s); T-n, transect number; LQPn, left quarterpoint, $n$th tree; ---, no data; RQPn, right quarterpoint, nth tree]

\begin{tabular}{|c|c|c|c|c|c|}
\hline $\begin{array}{c}\text { Tree } \\
\text { position }\end{array}$ & Species code & Scientific name & Common name & $\begin{array}{l}\text { Distance } \\
\text { from } \\
\text { pivot } \\
\text { point }(\mathrm{m})\end{array}$ & $\begin{array}{c}\text { Trunk diameter at } \\
\text { breast } \\
\text { height }(\mathrm{m})\end{array}$ \\
\hline T-7 LQP1 & Pasture & no tree & --- & --- & --- \\
\hline T-7 LQP2 & Pasture & no tree & -- & --- & -- \\
\hline T-7 LQP3 & Pasture & no tree & --- & --- & --- \\
\hline T-7 LQP4 & Pasture & no tree & --- & -- & -- \\
\hline T-7 RQP1 & ACNE & Acer negundo & boxelder & 3.1 & 0.09 \\
\hline T-7 RQP2 & FRAM & Fraxinus americana & white ash & 2.2 & 0.32 \\
\hline T-7 RQP3 & RUFL & Rubus flagellaris & prickly dewberry & 0.5 & multiple stems \\
\hline T-7 RQP4 & $\mathrm{ACNE}$ & Acer negundo & boxelder & 1.7 & 0.02 \\
\hline T-8 LQP1 & LIST & Liquidambar styraciflua & sweetgum & 4.7 & 0.54 \\
\hline T-8 LQP2 & $\mathrm{ACNE}$ & Acer negundo & boxelder & 3.8 & 0.05 \\
\hline T-8 LQP3 & LIST & Liquidambar styraciflua & sweetgum & 3.8 & 0.02 \\
\hline T-8 LQP4 & RUFL & Rubus flagellaris & prickly dewberry & 0.5 & multiple stems \\
\hline T-8 RQP1 & FRAM & Fraxinus americana & white ash & 9.1 & 0.25 \\
\hline T-8 RQP2 & PLOX & Platanus occidentalis & sycamore & 4.1 & 0.45 \\
\hline T-8 RQP3 & CACA & Carpinus caroliniana & ironwood & 4.8 & 0.17 \\
\hline T-8 RQP4 & PRSE & Prunus serotina & black cherry & 3.1 & 0.11 \\
\hline T-9 LQP1 & LIST & Liquidambar styraciflua & sweetgum & 1.6 & 0.12 \\
\hline T-9 LQP2 & LIST & Liquidambar styraciflua & sweetgum & 3 & 0.47 \\
\hline T-9 LQP3 & FRAM & Fraxinus americana & white ash & 5.2 & 0.18 \\
\hline T-9 LQP4 & $\mathrm{CACA}$ & Carpinus caroliniana & ironwood & 5.5 & 0.09 \\
\hline T-9 RQP1 & PRSE & Prunus serotina & black cherry & 3.1 & 0.16 \\
\hline T-9 RQP2 & FRAM & Fraxinus americana & white ash & 1.9 & 0.05 \\
\hline T-9 RQP3 & CACA & Carpinus caroliniana & ironwood & 1.9 & 0.05 \\
\hline T-9 RQP4 & CACA & Carpinus caroliniana & ironwood & 1.4 & 0.04 \\
\hline T-10 LQP1 & BENI & Betula nigra & river birch & 4.6 & 0.41 \\
\hline T-10 LQP2 & PRSE & Prunus serotina & black cherry & 3.1 & 0.20 \\
\hline T-10 LQP3 & FRAM & Fraxinus americana & white ash & 10.1 & 0.40 \\
\hline T-10 LQP4 & $\mathrm{CACA}$ & Carpinus caroliniana & ironwood & 7 & 0.08 \\
\hline T-10 RQP1 & QUAL & Quercus alha & white oak & 2.8 & 0.06 \\
\hline T-10 RQP2 & FRAM & Fraxinus americana & white ash & 4 & 0.11 \\
\hline T-10 RQP3 & FRAM & Fraxinus americana & white ash & 3.1 & 0.26 \\
\hline T-10 RQP4 & FRAM & Fraxinus americana & white ash & 4.6 & 0.28 \\
\hline T-11 LQP1 & $\mathrm{ACNE}$ & Acernegundo & boxelder & 8.7 & 0.24 \\
\hline T-11 LQP2 & FRAM & Fraxinus americana & white ash & 2.6 & 0.03 \\
\hline T-11 LQP3 & FRAM & Fraxinus americana & white ash & 1.2 & 0.04 \\
\hline T-11 LQP4 & LIST & Liquidambar styraciflua & sweetgum & 3.5 & 0.35 \\
\hline T-11 RQP1 & FRAM & Fraxinus americana & white ash & 4.6 & 0.30 \\
\hline T-11 RQP2 & $\mathrm{ACNE}$ & Acer negundo & boxelder & 5.7 & 0.27 \\
\hline T-11 RQP3 & CACA & Carpinus caroliniana & ironwood & 2 & 0.02 \\
\hline T-11 RQP4 & COFL & Cornus florida & flowering dogwood & 7 & 0.03 \\
\hline T-12 LQP1 & CACA & Carpinus caroliniana & ironwood & 1.8 & 0.24 \\
\hline T-12 LQP2 & $\mathrm{CACA}$ & Carpinus caroliniana & ironwood & 3.9 & 0.20 \\
\hline T-12 LQP3 & $\mathrm{ACNE}$ & Acer negundo & boxelder & 4.5 & 0.26 \\
\hline T-12 LQP4 & QUNI & Quercus nigra & water oak & 5.9 & 0.07 \\
\hline T-12 RQPI & FRAM & Fraxinus americana & white ash & 1.7 & 0.12 \\
\hline T-12 RQP2 & FRAM & Fraxinus americana & white ash & 2.3 & 0.36 \\
\hline T-12 RQP3 & FRAM & Fraxinus americana & white ash & 4 & 0.32 \\
\hline T-12 RQP4 & ACNE & Acernegundo & boxelder & 3.2 & 0.18 \\
\hline
\end{tabular}


Table 11. Riparian-zone trees, Brushy Creek near Pelham, S.C., USGS 021603257 (reach 4)

[m, meter(s); T-n, transect number; LQPn, left quarterpoint, nth tree; RQPn, right quarterpoint, nth tree; NI, not identified; ---, no data]

\begin{tabular}{|c|c|c|c|c|c|}
\hline $\begin{array}{c}\text { Tree } \\
\text { position }\end{array}$ & $\begin{array}{l}\text { Species } \\
\text { code }\end{array}$ & Scientific name & Common name & $\begin{array}{l}\text { Distance } \\
\text { from pivot } \\
\text { point (m) }\end{array}$ & $\begin{array}{l}\text { Trunk diameter at } \\
\text { breast height }(\mathrm{m})\end{array}$ \\
\hline T-13 LQP1 & PLOC & Platanus occidentalis & sycamore & 5.1 & 0.38 \\
\hline T-13 LQP2 & LIST & Liquidambar styraciflua & sweetgum & 17.2 & 0.43 \\
\hline T-13 LQP3 & BENI & Betula nigra & river birch & 10.7 & 0.10 \\
\hline T-13 LQP4 & LITU & Liriodendron tulipifera & tulip tree & 5.2 & 0.25 \\
\hline T-13 RQP1 & ACNE & Acernegundo & boxelder & 1.1 & 0.14 \\
\hline T-13 RQP2 & PLOC & Platanus occidentalis & sycamore & 1.6 & 0.61 \\
\hline T-13 RQP3 & PLOC & Platanus occidentalis & sycamore & 4.4 & 0.04 \\
\hline T-13 RQP4 & ACNE & Acernegundo & boxelder & 1.2 & 0.07 \\
\hline T-14 LQP1 & ACNE & Acernegundo & boxelder & 1.8 & 2.90 \\
\hline T-14 LQP2 & BENI & Betula nigra & river birch & 2.6 & 0.35 \\
\hline T-14 LQP3 & ACNE & Acer negundo & boxelder & 3.3 & 0.07 \\
\hline T-14 LQP4 & ACNE & Acer negundo & boxelder & 2.4 & 0.04 \\
\hline T-14 RQP1 & ACNE & Acernegundo & boxelder & 6.2 & 0.02 \\
\hline T-14 RQP2 & FRSP & Fraxinus sp. & ash & 5.1 & 0.17 \\
\hline T-14 RQP3 & ACNE & Acer negundo & boxelder & 5.8 & 0.2 \\
\hline T-14 RQP4 & ACNE & Acernegundo & boxelder & 4.5 & 0.07 \\
\hline T-15 LQP1 & LITU & Liriodendron tulipifera & tulip tree & 7.8 & 0.23 \\
\hline T-15 LQP2 & ACNE & Acernegundo & boxelder & 1.6 & 0.23 \\
\hline T-15 LQP3 & NI & -- & tree & 4.1 & 0.07 \\
\hline T-15 LQP4 & GLTR & Gleditsia triacanthos & honey locust & 3.6 & 0.15 \\
\hline T-15 RQP1 & ACNE & Acer negundo & boxelder & 2.2 & 0.03 \\
\hline T-15 RQP2 & ACNE & Acer negundo & boxelder & 3.5 & 0.03 \\
\hline T-15 RQP3 & ACNE & Acer negundo & boxelder & 5.8 & 0.38 \\
\hline T-15 RQP4 & GLTR & Gleditsia triacanthos & honey locust & 4.1 & 0.05 \\
\hline T-16 LQP1 & LIST & Liquidambar styraciflua & sweetgum & 17.4 & 0.62 \\
\hline T-16 LQP2 & ACNE & Acer negundo & boxelder & 5.5 & 0.03 \\
\hline T-16 LQP3 & PRSE & Prunus serotina & black cherry & 4 & 0.22 \\
\hline T-16 LQP4 & NI & -- & tree & 9.2 & 0.03 \\
\hline T-16 RQP1 & NI & -- & tree & 3.1 & 0.03 \\
\hline T-16 RQP2 & ACNE & Acer negundo & boxelder & 1.3 & 0.07 \\
\hline T-16 RQP3 & ACNE & Acernegundo & boxelder & 5 & 0.46 \\
\hline T-16 RQP4 & ACNE & Acer negundo & boxelder & 1.3 & 0.07 \\
\hline T-17 LQP1 & LIST & Liquidambar styraciflua & sweetgum & 2.5 & 0.52 \\
\hline T-17 LQP2 & LIST & Liquidambar styraciflua & sweetgum & 3.7 & 0.08 \\
\hline T-17 LQP3 & LIST & Liquidambar styraciflua & sweetgum & 3.9 & 0.08 \\
\hline T-17 LQP4 & LIST & Liquidambar styraciflua & sweetgum & 3 & 0.31 \\
\hline T-17 RQP1 & LIST & Liquidambar styraciflua & sweetgum & 4.8 & 0.10 \\
\hline T-17 RQP2 & GLTR & Gleditsia triacanthos & honey locust & 4.8 & 0.06 \\
\hline T-17 RQP3 & MALUS & Malus sp. & crab apple & 8.8 & 0.18 \\
\hline T-17 RQP4 & NI & --- & tree & 8 & 0.03 \\
\hline T-18 LQP1 & QUNI & Quercus nigra & water oak & 6.2 & 0.56 \\
\hline T-18 LQP2 & LIST & Liquidambar styraciflua & sweetgum & 5.1 & 0.38 \\
\hline T-18 LQP3 & BENI & Betula nigra & river birch & 2.4 & 0.49 \\
\hline T-18 LQP4 & LIST & Liquidambar styraciflua & sweetgum & 6 & 0.37 \\
\hline T-18 RQP1 & ACNE & Acer negundo & boxelder & 2.2 & 0.46 \\
\hline T-18 RQP2 & NI & $-\ldots$ & shrub & 3.8 & 0.03 \\
\hline T-18 RQP3 & NI & --- & shrub & 1.5 & 0.11 \\
\hline T-18 RQP4 & ACNE & Acernegundo & boxelder & 7.2 & 0.19 \\
\hline
\end{tabular}


Table 12. Riparian-zone trees, Indian Creek above Newberry, S.C., USGS 021607224 (reach 5) [m, meter(s); T- $n$, transect number; LQPn, left quarterpoint; $n$th tree; RQPn, right quarterpoint, $n$th tree]

\begin{tabular}{|c|c|c|c|c|c|}
\hline Tree position & Species code & Scientific name & Common name & $\begin{array}{l}\text { Distance } \\
\text { from pivot } \\
\text { point (m) }\end{array}$ & $\begin{array}{l}\text { Trunk diameter at } \\
\text { breast height (m) }\end{array}$ \\
\hline T-19 LQPI & LITU & Liriodendron tulipifera & tulip poplar & 2.5 & 0.49 \\
\hline T-19 LQP2 & LIST & Liquidambar styraciflua & sweet gum & 2.0 & 0.09 \\
\hline T-19 LQP3 & CACA & Carpinus caroliniana & ironwood & 2.4 & 0.07 \\
\hline T-19 LQP4 & ACRU & Acer rubrum & red maple & 2.2 & 0.07 \\
\hline T-19 RQP1 & CACA & Carpinus caroliniana & ironwood & 1.0 & 0.15 \\
\hline T-19 RQP2 & CACA & Carpinus caroliniana & ironwood & 1.0 & 0.16 \\
\hline T-19 RQP3 & CACA & Carpinus caroliniana & ironwood & 4.9 & 0.11 \\
\hline T-19 RQP4 & CACA & Carpinus caroliniana & ironwood & 4.9 & 0.16 \\
\hline T-20 LQP1 & CACA & Carpinus caroliniana & ironwood & 2.5 & 0.08 \\
\hline T-20 LQP2 & CACA & Carpinus caroliniana & ironwood & 3.1 & 0.14 \\
\hline T-20 LQP3 & ACRU & Acer rubrum & red maple & 1.8 & 0.04 \\
\hline T-20 LQP4 & LIST & Liquidambar styraciflua & sweetgum & 5.3 & 0.13 \\
\hline T-20 RQP1 & LIST & Liquidambar styraciflua & sweetgum & 1.8 & 0.44 \\
\hline T-20 RQP2 & $\mathrm{ACRU}$ & Acer rubrum & red maple & 0.9 & 0.30 \\
\hline T-20 RQP3 & CACA & Carpinus caroliniana & ironwood & 2.6 & 0.16 \\
\hline T-20 RQP4 & $\mathrm{ACRU}$ & Acer rubrum & red maple & 1.2 & 0.40 \\
\hline T-21 LQP1 & BENI & Betula nigra & river birch & 2.5 & 0.46 \\
\hline T-21 LQP2 & CACA & Carpinus caroliniana & ironwood & 2.8 & 0.05 \\
\hline T-21 LQP3 & CACA & Carpinus caroliniana & ironwood & 2.9 & 0.12 \\
\hline T-21 LQP4 & CACA & Carpinus caroliniana & ironwood & 2.9 & 0.09 \\
\hline T-21 RQP1 & LIST & Liquidambar styraciflua & sweetgum & 2.5 & 0.18 \\
\hline $\mathrm{T}-21 \mathrm{RQP} 2$ & ACRU & Acer rubrum & red maple & 2.0 & 0.09 \\
\hline T-21 RQP3 & BENI & Betula nigra & river birch & 3.5 & 0.30 \\
\hline T-21 RQP4 & BENI & Betula nigra & river birch & 2.8 & 0.41 \\
\hline T-22 LQP1 & PLOX & Platanus occidentalis & sycamore & 2.8 & 0.16 \\
\hline T-22 LQP2 & LIST & Liquidambar styraciflua & sweetgum & 2.5 & 0.05 \\
\hline T-22 LQP3 & LIST & Liquidambar styraciflua & sweetgum & 2.9 & 0.14 \\
\hline T-22 LQP4 & LIST & Liquidambar styraciflua & sweetgum & 2.6 & 0.14 \\
\hline T-22 RQP1 & LIST & Liquidambar styraciflua & sweetgum & 2.2 & 0.12 \\
\hline T-22 RQP2 & CACA & Carpinus caroliniana & ironwood & 3.3 & 0.16 \\
\hline T-22 RQP3 & COFL & Cornus florida & flowering dogwood & 4.0 & 0.11 \\
\hline T-22 RQP4 & LIST & Liquidambar styraciflua & sweetgum & 1.9 & 0.04 \\
\hline T-23 LQP1 & CACA & Carpinus caroliniana & ironwood & 1.3 & 0.15 \\
\hline T-23 LQP2 & BENI & Betula nigra & river birch & 5.6 & 0.23 \\
\hline T-23 LQP3 & LIST & Liquidambar styraciflua & sweetgum & 2.2 & 0.21 \\
\hline T-23 LQP4 & QUAL & Quercus alba & white oak & 7.3 & 0.08 \\
\hline T-23 RQPI & BENI & Betula nigra & river birch & 4.7 & 0.32 \\
\hline T-23 RQP2 & ACRU & Acer rubrum & red maple & 2.4 & 0.24 \\
\hline T-23 RQP3 & LIST & Liquidambar styraciflua & sweetgum & 5.3 & 0.23 \\
\hline T-23 RQP4 & BENI & Betula nigra & river birch & 3.2 & 0.35 \\
\hline T-24 LQP1 & QUAL & Quercus alba & white oak & 2.5 & 0.58 \\
\hline T-24 LQP2 & LIST & Liquidambar styraciflua & sweetgum & 0.8 & 0.06 \\
\hline T-24 LQP3 & LIST & Liquidambar styraciflua & sweetgum & 2.7 & 0.05 \\
\hline T-24 LQP4 & COFL & Cornus florida & flowering dogwood & 1.5 & 0.06 \\
\hline T-24 RQP1 & COFL & Cornus florida & flowering dogwood & 2.4 & 0.07 \\
\hline T-24 RQP2 & CACA & Carpinus caroliniana & ironwood & 4.0 & 0.04 \\
\hline T-24 RQP3 & CACA & Carpinus caroliniana & ironwood & 6.4 & 0.17 \\
\hline T-24 RQP4 & CACA & Carpinus caroliniana & ironwood & 4.5 & 0.21 \\
\hline
\end{tabular}


Table 13. Riparian-zone trees, Indian Creek above Newberry, S.C., USGS 021607224 (reach 6)

[m, meter(s) T-n, transect number; LQPn, left quarterpoint, nth tree; RQPn, right quarterpoint, nth tree; NI, not identified; ---, no data]

\begin{tabular}{|c|c|c|c|c|c|}
\hline Tree position & Species code & Scientific name & Common name & $\begin{array}{l}\text { Distance } \\
\text { from pivot } \\
\text { point (m) }\end{array}$ & $\begin{array}{l}\text { Trunk diameter at } \\
\text { breast height (m) }\end{array}$ \\
\hline T-25 LQP1 & CACA & Carpinus caroliniana & ironwood & 6.3 & 0.05 \\
\hline T-25 LQP2 & CACA & Carpinus caroliniana & ironwood & 4.0 & 0.12 \\
\hline T-25 LQP3 & PITA & Pinus taeda & loblolly pine & 4.7 & 0.26 \\
\hline T-25 LQP4 & CACA & Carpinus caroliniana & ironwood & 4.8 & 0.08 \\
\hline T-25 RQP1 & QUNI & Quercus nigra & water oak & 4.2 & 0.30 \\
\hline T-25 RQP2 & PITA & Pinus taeda & loblolly pine & 7.0 & 0.42 \\
\hline T-25 RQP3 & ACRU & Acer rubrum & red maple & 6.5 & 0.07 \\
\hline T-25 RQP4 & $\mathrm{CACA}$ & Carpinus caroliniana & ironwood & 4.2 & 0.11 \\
\hline T-26 LQP1 & $\mathrm{CACA}$ & Carpinus caroliniana & ironwood & 3.4 & 0.05 \\
\hline T-26 LQP2 & CACA & Carpinus caroliniana & ironwood & 2.7 & 0.09 \\
\hline T-26 LQP3 & COFL & Cornus florida & flowering dogwood & 3.1 & 0.05 \\
\hline T-26 LQP4 & COFL & Cornus florida & flowering dogwood & 2.8 & 0.03 \\
\hline T-26 RQP1 & CACA & Carpinus caroliniana & ironwood & 3.7 & 0.14 \\
\hline T-26 RQP2 & CACA & Carpinus caroliniana & ironwood & 3.8 & 0.12 \\
\hline T-26 RQP3 & $\mathrm{CACA}$ & Carpinus caroliniana & ironwood & 4.2 & 0.13 \\
\hline T-26 RQP4 & OSVI & Ostrya virginiana & hop hornbeam & 3.8 & 0.12 \\
\hline T-27 LQP1 & CACA & Carpinus caroliniana & ironwood & 2.1 & 0.04 \\
\hline T-27 LQP2 & QUNI & Quercus nigra & water oak & 3.3 & 0.16 \\
\hline T-27 LQP3 & LITU & Liriodendron tulipifera & tulip poplar & 3.7 & 0.46 \\
\hline T-27 LQP4 & CACA & Carpinus caroliniana & ironwood & 3.6 & 0.11 \\
\hline T-27 RQP1 & CACA & Carpinus caroliniana & ironwood & 4.5 & 0.13 \\
\hline T-27 RQP2 & NI & --- & tree & 4.0 & 0.33 \\
\hline T-27 RQP3 & $\mathrm{CACA}$ & Carpinus caroliniana & ironwood & 4.0 & 0.08 \\
\hline T-27 RQP4 & CACA & Carpinus caroliniana & ironwood & 4.3 & 0.03 \\
\hline T-28 LQP1 & LIST & Liquidambar styraciflua & sweetgum & 1.5 & 0.15 \\
\hline T-28 LQP2 & CACA & Carpinus caroliniana & ironwood & 4.1 & 0.18 \\
\hline T-28 LQP3 & QURU & Quercus rubra & red oak & 4.8 & 0.56 \\
\hline T-28 LQP4 & LIST & Liquidambar styraciflua & sweetgum & 2.8 & 0.18 \\
\hline T-28 RQP1 & $\mathrm{CACA}$ & Carpinus caroliniana & ironwood & 2.0 & 0.08 \\
\hline T-28 RQP2 & $\mathrm{CACA}$ & Carpinus caroliniana & ironwood & 3.0 & 0.11 \\
\hline T-28 RQP3 & QUNI & Quercus nigra & water oak & 2.0 & 0.28 \\
\hline T-28 RQP4 & CACA & Carpinus caroliniana & ironwood & 1.7 & 0.07 \\
\hline T-29 LQP1 & CACA & Carpinus caroliniana & ironwood & 2.5 & 0.06 \\
\hline T-29 LQP2 & BENI & Betula nigra & river birch & 2.4 & 0.24 \\
\hline T-29 LQP3 & LIST & Liquidambar styraciflua & sweetgum & 5.2 & 0.45 \\
\hline T-29 LQP4 & BENI & Betula nigra & river birch & 1.5 & 0.43 \\
\hline T-29 RQP1 & COFL & Cornus florida & flowering dogwood & 1.3 & 0.05 \\
\hline T-29 RQP2 & LIST & Liquidambar styraciflua & sweetgum & 3.8 & 0.10 \\
\hline T-29 RQP3 & LIST & Liquidambar styraciflua & sweetgum & 3.3 & 0.06 \\
\hline T-29 RQP4 & CACA & Carpinus caroliniana & ironwood & 4.8 & 0.05 \\
\hline T-30 LQP1 & $\mathrm{CACA}$ & Carpinus caroliniana & ironwood & 2.4 & 0.04 \\
\hline T-30 LQP2 & BENI & Betula nigra & river birch & 3.5 & 0.41 \\
\hline T-30 LQP3 & BENI & Betula nigra & river birch & 3.8 & 0.35 \\
\hline T-30 LQP4 & BENI & Betula nigra & river birch & 5.9 & 0.32 \\
\hline T-30 RQP1 & LIST & Liquidambar styraciflua & sweetgum & 1.2 & 0.11 \\
\hline T-30 RQP2 & COFL & Cornus florida & flowering dogwood & 2.6 & 0.05 \\
\hline T-30 RQP3 & LIST & Liquidambar styraciflua & sweetgum & 3.7 & 0.20 \\
\hline $\mathrm{T}-30 \mathrm{RQP} 4$ & CACA & Carpinus caroliniana & ironwood & 5.6 & 0.06 \\
\hline
\end{tabular}


Table 14. Riparian-zone trees, Indian Creek above Newberry, S.C., USGS 021607224 (reach 7) [m, meter(s); T-n, transect number; LQPn, left quarterpoint, $n$th tree; RQPn, right quarterpoint, $n$th tree]

\begin{tabular}{|c|c|c|c|c|c|}
\hline Tree position & $\begin{array}{l}\text { Species } \\
\text { code }\end{array}$ & Scientific name & Common name & $\begin{array}{c}\text { Distance } \\
\text { from pivot } \\
\text { point }(m)\end{array}$ & $\begin{array}{l}\text { Trunk diameter at } \\
\text { breast height }(\mathrm{m})\end{array}$ \\
\hline T-31 LQP1 & QUNI & Quercus nigra & water oak & 0.5 & 0.11 \\
\hline T-31 LQP2 & BENI & Betula nigra & river birch & 2.9 & 0.54 \\
\hline T-31 LQP3 & JUVI & Juniperus virginiana & eastern redcedar & 2.2 & 0.08 \\
\hline T-31 LQP4 & LIST & Liquidambar styraciflua & sweetgum & 0.8 & 0.12 \\
\hline T-31 RQP1 & BENI & Betula nigra & river birch & 4.0 & 0.08 \\
\hline T-31 RQP2 & BENI & Betula nigra & river birch & 2.2 & 0.25 \\
\hline T-31 RQP3 & CACA & Carpinus caroliniana & ironwood & 0.6 & 0.03 \\
\hline T-31 RQP4 & BENI & Betula nigra & river birch & 1.5 & 0.03 \\
\hline T-32 LQP1 & ULAL & Ulmus alata & winged elm & 4.2 & 0.09 \\
\hline T-32 LQP2 & BENI & Betula nigra & river birch & 2.9 & 0.51 \\
\hline T-32 LQP3 & LIST & Liquidambar styraciflua & sweetgum & 3.2 & 0.60 \\
\hline T-32 LQP4 & ACRU & Acer rubrum & red maple & 4.8 & 0.20 \\
\hline T-32 RQP1 & CACA & Carpinus caroliniana & ironwood & 3.1 & 0.04 \\
\hline T-32 RQP2 & JUVI & Juniperus virginiana & eastern redcedar & 3.4 & 0.06 \\
\hline T-32 RQP3 & LIST & Liquidambar styraciflua & sweetgum & 0.9 & 0.06 \\
\hline T-32 RQP4 & ACRU & Acer rubrum & red maple & 2 & 0.04 \\
\hline T-33 LQP1 & CACA & Carpinus caroliniana & ironwood & 0.5 & 0.05 \\
\hline T-33 LQP2 & BENI & Betula nigra & river birch & 6.1 & 0.22 \\
\hline T-33 LQP3 & ACRU & Acer rubrum & red maple & 3.2 & 0.33 \\
\hline T-33 LQP4 & BENI & Betula nigra & river birch & 4.4 & 0.18 \\
\hline T-33 RQP1 & QUMI & Quercus michauxii & basket oak & 1.1 & 0.06 \\
\hline T-33 RQP2 & COFL & Cornus florida & flowering dogwood & 2.1 & 0.03 \\
\hline T-33 RQP3 & COFL & Cornus florida & flowering dogwood & 2.5 & 0.10 \\
\hline T-33 RQP4 & BENI & Betula nigra & river birch & 1.2 & 0.12 \\
\hline T-34 LQP1 & PLOC & Platanus occidentalis & sycamore & 1.8 & 0.27 \\
\hline T-34 LQP2 & CACA & Carpinus caroliniana & ironwood & 6.5 & 0.19 \\
\hline T-34 LQP3 & ACRU & Acer rubrum & red maple & 4.1 & 0.12 \\
\hline T-34 LQP4 & ACRU & Acer rubrum & red maple & 1.4 & 0.54 \\
\hline T-34 RQP1 & QUNI & Quercus nigra & water oak & 2.2 & 0.14 \\
\hline T-34 RQP2 & CACA & Carpinus caroliniana & ironwood & 2.8 & 0.03 \\
\hline T-34 RQP3 & CACA & Carpinus caroliniana & ironwood & 1.9 & 0.17 \\
\hline T-34 RQP4 & LIST & Liquidambar styraciflua & sweetgum & 2 & 0.17 \\
\hline T-35 LQP1 & ACRU & Acer rubrum & red maple & 0.2 & 0.10 \\
\hline T-35 LQP2 & QUMI & Quercus michauxii & basket oak & 5.2 & 0.12 \\
\hline T-35 LQP3 & LITU & Liriodendron tulipifera & tulip tree & 1.6 & 0.10 \\
\hline $\mathrm{T}-35 \mathrm{LQP} 4$ & CACA & Carpinus caroliniana & ironwood & 0.3 & 0.10 \\
\hline T-35 RQP1 & ACNE & Acer negundo & box elder & 2.9 & 0.15 \\
\hline $\mathrm{T}-35 \mathrm{RQP} 2$ & PLOC & Platanus occidentalis & sycamore & 1.2 & 0.44 \\
\hline $\mathrm{T}-35 \mathrm{RQP} 3$ & BENI & Betula nigra & river birch & 1.4 & 0.49 \\
\hline T-35 RQP4 & CACA & Carpinus caroliniana & ironwood & 0.8 & 0.07 \\
\hline T-36 LQP1 & CACA & Carpinus caroliniana & ironwood & 2.5 & 0.05 \\
\hline T-36 LQP2 & BENI & Betula nigra & river birch & 3.9 & 0.49 \\
\hline T-36 LQP3 & CACA & Carpinus caroliniana & ironwood & 6.4 & 0.18 \\
\hline T-36 LQP4 & BENI & Betula nigra & river birch & 9.1 & 0.03 \\
\hline $\mathrm{T}-36 \mathrm{RQP1}$ & ACRU & Acer rubrum & red maple & 1.8 & 0.04 \\
\hline $\mathrm{T}-36 \mathrm{RQP} 2$ & ACRU & Acer rubrum & red maple & 3.5 & 0.35 \\
\hline $\mathrm{T}-36 \mathrm{RQP3}$ & BENI & Betula nigra & river birch & 1.4 & 0.34 \\
\hline T-36 RQP4 & CACA & Carpinus caroliniana & ironwood & 2.2 & 0.08 \\
\hline
\end{tabular}


Table 15. Riparian-zone trees, Gills Creek near Hopkins, S.C., USGS 02169595 (reach 8)

[ $m$, meter(s); T-n, transect number; LQPn, left quarterpoint, $n$th tree; RQPn, right quarterpoint, $n$th tree]

\begin{tabular}{|c|c|c|c|c|c|}
\hline Tree position & Species code & Scientific name & Common name & $\begin{array}{l}\text { Distance } \\
\text { from pivot } \\
\text { point (m) }\end{array}$ & $\begin{array}{l}\text { Trunk diameter at } \\
\text { breast height ( } \mathrm{m})\end{array}$ \\
\hline T-37 LQP1 & NYAQ & Nyssa aquatica & water tupelo & 3.7 & 0.31 \\
\hline T-37 LQP2 & CETE & Celtis tenuifolia & upland hackberry & 3.2 & 0.04 \\
\hline T-37 LQP3 & BRPA & Broussonetia papyrifera & paper mulberry & 2.4 & 0.10 \\
\hline T-37 LQP4 & CACA & Carpinus caroliniana & ironwood & 8.4 & 0.28 \\
\hline T-37 RQP1 & CACA & Carpinus caroliniana & ironwood & 2 & 0.16 \\
\hline T-37 RQP2 & LIST & Liquidambar styraciflua & sweetgum & 2.9 & 0.60 \\
\hline T-37 RQP3 & CACA & Carpinus caroliniana & ironwood & 2.3 & 0.07 \\
\hline T-37 RQP4 & PLAQ & Planera aquatica & water elm & 7.8 & 0.25 \\
\hline T-38 LQP1 & CACA & Carpinus caroliniana & ironwood & 3.1 & 0.10 \\
\hline T-38 LQP2 & QUMI & Quercus michauxii & chinquapin oak & 2.6 & 0.43 \\
\hline T-38 LQP3 & QUNI & Quercus nigra & water oak & 2.1 & 0.23 \\
\hline T-38 LQP4 & PLAQ & Planera aquatica & water elm & 3.9 & 0.06 \\
\hline T-38 RQP1 & CACA & Carpinus caroliniana & ironwood & 1.9 & 0.09 \\
\hline T-38 RQP2 & ACRU & Acer rubrum & red maple & 3.8 & 0.21 \\
\hline T-38 RQP3 & CACA & Carpinus caroliniana & ironwood & 2 & 0.06 \\
\hline T-38 RQP4 & ACRU & Acer rubrum & red maple & 2.4 & 0.09 \\
\hline T-39 LQP1 & CACA & Carpinus caroliniana & ironwood & 2.1 & 0.15 \\
\hline T-39 LQP2 & CACA & Carpinus caroliniana & ironwood & 2.6 & 0.28 \\
\hline T-39 LQP3 & QUNI & Quercus nigra & water oak & 2.8 & 0.34 \\
\hline T-39 LQP4 & CACA & Carpinus caroliniana & ironwood & 1.7 & 0.07 \\
\hline T-39 RQP1 & BENI & Betula nigra & river birch & 2 & 0.72 \\
\hline T-39 RQP2 & ACRU & Acer rubrum & red maple & 2.9 & 0.24 \\
\hline T-39 RQP3 & LIST & Liquidambar styraciflua & sweetgum & 3.8 & 0.17 \\
\hline T-39 RQP4 & LIST & Liquidambar styraciflua & sweetgum & 4.7 & 0.31 \\
\hline T-40 LQP1 & ILMO & Ilex montana & largeleaf holly & 1.2 & 0.06 \\
\hline T-40 LQP2 & CAPA & Carya pallida & sand hickory & 1.6 & 0.33 \\
\hline T-40 LQP3 & LIST & Liquidambar styraciflua & sweetgum & 2.1 & 0.10 \\
\hline T-40 LQP4 & CACA & Carpinus caroliniana & ironwood & 3.6 & 0.34 \\
\hline T-40 RQP1 & CACA & Carpinus caroliniana & ironwood & 2.8 & 0.16 \\
\hline T-40 RQP2 & OSVI & Ostrya virginiana & hop hornbeam & 4.3 & 0.47 \\
\hline T-40 RQP3 & ACRU & Acer rubrum & red maple & 3.2 & 0.18 \\
\hline T-40 RQP4 & CAPA & Carya pallida & sand hickory & 3 & 0.27 \\
\hline T-41 LQP1 & CATO & Carya tomentosa & mockernut hickory & 3.4 & 0.36 \\
\hline T-41 LQP2 & PLAQ & Planera aquatica & water elm & 4.5 & 0.54 \\
\hline T-41 LQP3 & CATO & Carya glabra & pignut hickory & 4.2 & 0.08 \\
\hline T-41 LQP4 & CACA & Carpinus caroliniana & ironwood & 4.5 & 0.20 \\
\hline T-41 RQP1 & ACRU & Acer rubrum & red maple & 4.2 & 0.26 \\
\hline T-41 RQP2 & ACRU & Acer rubrum & red maple & 6.3 & 0.26 \\
\hline T-41 RQP3 & ACRU & Acer rubrum & red maple & 3.9 & 0.38 \\
\hline T-41 RQP4 & CACA & Carpinus caroliniana & ironwood & 3.7 & 0.23 \\
\hline T-42 LQP1 & ACRU & Acer rubrum & red maple & 2.9 & 0.64 \\
\hline T-42 LQP2 & ACRU & Acer rubrum & red maple & 2.8 & 0.18 \\
\hline T-42 LQP3 & QUPH & Quercus phellos & willow oak & 3 & 0.38 \\
\hline T-42 LQP4 & ACRU & Acer rubrum & red maple & 5.7 & 0.27 \\
\hline T-42 RQP1 & QUNI & Quercus nigra & water oak & 4.6 & 0.52 \\
\hline T-42 RQP2 & CACA & Carpinus caroliniana & ironwood & 1.5 & 0.12 \\
\hline T-42 RQP3 & CACA & Carpinus caroliniana & ironwood & 6.1 & 0.07 \\
\hline T-42 RQP4 & CACA & Carpinus caroliniana & ironwood & 3.5 & 0.18 \\
\hline
\end{tabular}


Table 16. Riparian-zone trees, Gills Creek at Columbia, S.C., USGS 02169570 (reach 9)

[m, meter(s); T-n, transect number; LQPn, left quarterpoint, $n$th tree; RQPn, right quarterpoint, $n$th tree; NI, not identified; --, no data

\begin{tabular}{|c|c|c|c|c|c|}
\hline Tree position & Species code & Scientific name & Common name & $\begin{array}{l}\text { Distance } \\
\text { from pivot } \\
\text { point (m) }\end{array}$ & $\begin{array}{l}\text { Trunk diameter at } \\
\text { breast height }(\mathrm{m})\end{array}$ \\
\hline T-43 LQP2 & ACRU & Acer rubrum & red maple & 3.3 & 0.23 \\
\hline T-43 LQP3 & LITU & Liriodendron tulipifera & tulip tree & 3.6 & 0.24 \\
\hline T-43 LQP4 & LITU & Liriodendron tulipifera & tulip tree & 3.1 & 0.29 \\
\hline T-43 RQPI & ACRU & Acer rubrum & red maple & 2.3 & 0.35 \\
\hline T-43 RQP2 & NI & -- & shrub & 3.6 & 0.04 \\
\hline T-43 RQP3 & NI & --- & shrub & 2.1 & 0.02 \\
\hline T-43 RQP4 & NI & --- & shrub & 2.6 & 0.02 \\
\hline T-44 LQP1 & SMGL & Smilax glauca & glaucous greenbrier & 0 & multiple stems \\
\hline T-44 LQP2 & ACRU & Acer rubrum & red maple & 2.2 & 0.27 \\
\hline T-44 LQP3 & SMRO & Smilax glauca & glaucous greenbrier & 1.6 & multiple stems \\
\hline T-44 LQP4 & QUNI & Quercus nigra & water oak & 1.6 & 0.11 \\
\hline T-44 RQP1 & RHVE & Rhus vernix & poison sumac & 1 & multiple stems \\
\hline T-44 RQP2 & RUFL & Rubus flagellaris & prickly dewberry & 0.05 & 0.02 \\
\hline T-44 RQP3 & RUFL & Rubus flagellaris & prickly dewberry & 2 & multiple stems \\
\hline T-44 RQP4 & RUFL & Rubus flagellaris & prickly dewberry & 1 & multiple stems \\
\hline T-45 LQP1 & BENI & Betula nigra & river birch & 0.8 & 0.67 \\
\hline T-45 LQP2 & LIST & Liquidambar styraciflua & sweetgum & 0.3 & 1.80 \\
\hline T-45 LQP3 & QUNI & Quercus nigra & water oak & 5 & 0.68 \\
\hline T-45 LQP4 & LIST & Liquidambar styraciflua & sweetgum & 5.1 & 0.36 \\
\hline T-45 RQP1 & NI & --- & shrub & 2 & 0.02 \\
\hline T-45 RQP2 & NI & -- & shrub & 2.5 & 0.02 \\
\hline T-45 RQP3 & ACRU & Acer rubrum & red maple & 1.1 & 0.09 \\
\hline T-45 RQP4 & ACRU & Acer rubrum & red maple & 1 & 0.08 \\
\hline T-46 LQP1 & NI & --- & shrub & -- & --- \\
\hline T-46 LQP2 & NI & -- & shrub & --- & \\
\hline T-46 LQP3 & NI & -- & shrub & -- & -- \\
\hline T-46 LQP4 & NI & --- - & shrub & --- & -- \\
\hline T-46 RQP1 & ACRU & Acer rubrum & red maple & 1.4 & 0.05 \\
\hline T-46 RQP2 & QUNI & Quercus nigra & water oak & 2 & 0.12 \\
\hline T-46 RQP3 & LIST & Liquidambar styraciflua & sweetgum & 3.8 & 0.54 \\
\hline T-46 RQP4 & NI & --- & shrub & 4.6 & 0.02 \\
\hline T-47 LQP1 & LIST & Liquidambar styraciflua & sweetgum & 4.3 & 0.36 \\
\hline T-47 LQP2 & QUNI & Quercus nigra & water oak & 3.1 & 0.03 \\
\hline T-47 LQP3 & LIST & Liquidambar styraciflua & sweetgum & 4.3 & 0.52 \\
\hline T-47 LQP4 & NI & -- & tree & 2.6 & 0.06 \\
\hline T-47 RQP1 & ACNE & Acer negundo & box elder & 4 & 0.04 \\
\hline $\mathrm{T}-47 \mathrm{RQP} 2$ & BENI & Betula nigra & river birch & 4.4 & 0.05 \\
\hline T-47 RQP3 & LIST & Liquidambar styraciflua & sweetgum & 5.8 & 0.37 \\
\hline T-47 RQP4 & PEBO & Persea borbonia & redbay & 2.4 & 0.35 \\
\hline T-48 LQP1 & LIST & Liquidambar styraciflua & sweetgum & 1.4 & 0.05 \\
\hline T-48 LQP2 & ACRU & Acer rubrum & red maple & 2.9 & 0.10 \\
\hline T-48 LQP3 & QUNI & Quercus nigra & water oak & 2.2 & 0.10 \\
\hline T-48 LQP4 & QUNI & Quercus nigra & water oak & 0.7 & 0.12 \\
\hline T-48 RQP1 & FRAM & Fraxinus americana & white ash & 4.4 & 0.18 \\
\hline T-48 RQP2 & FRAM & Fraxinus americana & white ash & 6.05 & 0.29 \\
\hline T-48 RQP3 & QUNI & Quercus nigra & water oak & 1.8 & 0.08 \\
\hline T-48 RQP4 & LIST & Liquidambar styraciflua & sweetgum & 5 & 0.30 \\
\hline
\end{tabular}


Table 17. Riparian-zone trees, Gills Creek at Columbia, S.C., USGS 02169570 (reach 10)

[m, meter(s); T-n, transect number; LQPn, left quarterpoint, nth tree; RQPn, right quarterpoint, nth tree; NI, not identified; --, no data]

\begin{tabular}{|c|c|c|c|c|c|}
\hline Tree position & Specles code & Scientlfic name & Common name & $\begin{array}{c}\text { Distance } \\
\text { from pivot } \\
\text { point (m) }\end{array}$ & $\begin{array}{l}\text { Trunk diameter at } \\
\text { breast height }(m)\end{array}$ \\
\hline T-49 LQP1 & SANI & Salix nigra & black willow & 2.1 & 0.11 \\
\hline T-49 LQP2 & BENI & Betula nigra & river birch & 2.2 & 0.52 \\
\hline T-49 LQP3 & ACRU & Acer rubrum & red maple & 2.2 & 0.03 \\
\hline T-49 LQP4 & LIST & Liquidambar styraciflua & sweetgum & 2.3 & 0.09 \\
\hline T-49 RQPI & BENI & Betula nigra & river birch & 1.2 & 0.03 \\
\hline T-49 RQP2 & PRSE & Prunus serotina & black cherry & 4 & 0.30 \\
\hline T-49 RQP3 & PLAQ & Planera aquatica & water elm & 3.6 & 0.18 \\
\hline T-49 RQP4 & QUNI & Quercus nigra & water oak & 4.6 & 0.06 \\
\hline T-50 LQP1 & NI & 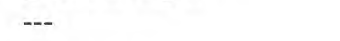 & shrub & 10.4 & 0.13 \\
\hline T-50 LQP2 & NI & --- & shrub & 3.3 & 0.03 \\
\hline T-50 LQP3 & NI & --- & shrub & 3.1 & 0.02 \\
\hline T-50 LQP4 & RUFL & Rubus flagellaris & prickly dewberry & 2 & patch \\
\hline T-50 RQP1 & \multicolumn{2}{|c|}{ Private property - no access } & -- & -- & -- \\
\hline T-50 RQP2 & \multicolumn{2}{|c|}{ Private property - no access } & -- & -- & -- \\
\hline T-50 RQP3 & \multicolumn{2}{|c|}{ Private property - no access } & -- & -- & -- \\
\hline T-50 RQP4 & \multicolumn{2}{|c|}{ Private property - no access } & --- & -- & --- \\
\hline T-51 LQP1 & LITU & Liriodendron tulipifera & tulip tree & 2.3 & 0.22 \\
\hline T-51 LQP2 & LITU & Liriodendron tulipifera & tulip tree & 2 & 0.25 \\
\hline T-51 LQP3 & SANI & Salix nigra & black willow & 8.8 & 0.10 \\
\hline T-51 LQP4 & ACRU & Acer rubrum & red maple & 10 & 0.16 \\
\hline T-51 RQP1 & \multicolumn{2}{|c|}{ Private property - no access } & --- & --- & --- \\
\hline T-51 RQP2 & \multicolumn{2}{|c|}{ Private property - no access } & --- & -- & -- \\
\hline T-51 RQP3 & \multicolumn{2}{|c|}{ Private property - no access } & -- & -- & $\cdots$ \\
\hline T-51 RQP4 & \multicolumn{2}{|c|}{ Private property - no access } & --- & -- & --- \\
\hline T-52 LQP1 & BENI & Betula nigra & river birch & 3.8 & 0.28 \\
\hline T-52 LQP2 & QUNI & Quercus nigra & water oak & 3.4 & 0.08 \\
\hline $\mathrm{T}-52 \mathrm{LQP} 3$ & QUNI & Quercus nigra & water oak & 2.5 & 0.08 \\
\hline T-52 LQP4 & QUNI & Quercus nigra & water oak & 2.1 & 0.13 \\
\hline T-52 RQP1 & \multicolumn{2}{|c|}{ Private property - no access } & -- & --- & -- \\
\hline T-52 RQP2 & \multicolumn{2}{|c|}{ Private property - no access } & --- & -- & -- \\
\hline $\mathrm{T}-52 \mathrm{RQP3}$ & \multicolumn{2}{|c|}{ Private property - no access } & --- & -- & -- \\
\hline T-52 RQP4 & \multicolumn{2}{|c|}{ Private property - no access } & --- & --- & -- \\
\hline T-53 LQP1 & RUFL & Rubus flagellaris & prickly dewberry & 1 & multiple stems \\
\hline T-53 LQP2 & NI & & tree & 4.8 & 0.03 \\
\hline T-53 LQP3 & QUNI & Quercus nigra & water oak & 8.2 & 0.06 \\
\hline T-53 LQP4 & LIST & Liquidambar styraciflua & sweetgum & 6.8 & 0.05 \\
\hline T-53 RQP1 & \multicolumn{2}{|c|}{ Private property - no access } & -- & --- & -- \\
\hline T-53 RQP2 & \multicolumn{2}{|c|}{ Private property - no access } & --- & -- & -- \\
\hline T-53 RQP3 & \multicolumn{2}{|c|}{ Private property - no access } & --- & -- & -- \\
\hline T-53 RQP4 & \multicolumn{2}{|c|}{ Private property - no access } & --- & --- & --- \\
\hline T-54 LQP1 & FRSP & Fraxinus species & ash species & 2 & 0.05 \\
\hline T-54 LQP2 & BENI & Betula nigra & river birch & 4.2 & 0.11 \\
\hline T-54 LQP3 & RUFL & Rubus flagellaris & prickly dewberry & 1 & multiple stems \\
\hline T-54 LQP4 & RUFL & Rubus flagellaris & prickly dewberry & 3 & multiple stems \\
\hline T-54 RQP1 & \multicolumn{2}{|c|}{ Private property - no access } & --- & -- & -- \\
\hline T-54 RQP2 & \multicolumn{2}{|c|}{ Private property - no access } & --- & -- & --- \\
\hline T-54 RQP3 & \multirow{2}{*}{\multicolumn{2}{|c|}{ Private property - no access }} & --- & -- & -- \\
\hline T-54 RQP4 & & & --- & --- & --- \\
\hline
\end{tabular}


Table 18. Riparian-zone trees, Myers Creek near Hopkins, S.C., USGS 02169660 (reach 11)

[m, meter(s); T-n, transect number; LQPn, left quarterpoint, $n$th tree; RQPn, right quarterpoint, $n$th tree]

\begin{tabular}{|c|c|c|c|c|c|}
\hline Tree position & Species code & Scientific name & Common name & $\begin{array}{l}\text { Distance } \\
\text { from pivot } \\
\text { point (m) }\end{array}$ & $\begin{array}{l}\text { Trunk diameter at } \\
\text { breast height (m) }\end{array}$ \\
\hline T-55 LQP1 & LIST & Liquidambar styraciflua & sweetgum & 1.6 & 0.50 \\
\hline T-55 LQP2 & ACRU & Acer rubrum & red maple & 7.2 & 0.46 \\
\hline T-55 LQP3 & LIST & Liquidambar styraciflua & sweetgum & 1.9 & 0.14 \\
\hline T-55 LQP4 & $\mathrm{ACRU}$ & Acer rubrum & red maple & 1.8 & 0.07 \\
\hline T-55 RQP1 & ACRU & Acer rubrum & red maple & 2.2 & 0.04 \\
\hline T-55 RQP2 & LIST & Liquidambar styraciflua & sweetgum & 2.6 & 0.14 \\
\hline T-55 RQP3 & ACRU & Acer rubrum & red maple & 3.6 & 0.08 \\
\hline T-55 RQP4 & ILDE & Ilex decidua & possumhaw holly & 2.3 & 0.04 \\
\hline T-56 LQP1 & NYAQ & Nyssa aquatica & water tupelo & 1.2 & 0.45 \\
\hline T-56 LQP2 & ULAM & Ulmus americana & American elm & 1.6 & 0.16 \\
\hline T-56 LQP3 & LIST & Liquidambar styraciflua & sweetgum & 2.1 & 0.44 \\
\hline T-56 LQP4 & ULAM & Ulmus americana & American elm & 1.0 & 0.11 \\
\hline T-56 RQP1 & LIST & Liquidambar styraciflua & sweetgum & 3.8 & 0.32 \\
\hline T-56 RQP2 & LIST & Liquidambar styraciflua & sweetgum & 5.0 & 0.42 \\
\hline T-56 RQP3 & NYAQ & Nyssa aquatica & water tupelo & 3.3 & 0.38 \\
\hline T-56 RQP4 & ILDE & Ilex decidua & possumhaw holly & 2.3 & 0.04 \\
\hline T-57 LQP1 & NYSY & Nyssa sylvatica & sourgum & 1.6 & 0.06 \\
\hline T-57 LQP2 & NYAQ & Nyssa aquatica & water tupelo & 7.3 & 0.23 \\
\hline T-57 LQP3 & ACRU & Acer rubrum & red maple & 1.3 & 0.11 \\
\hline T-57 LQP4 & ACRU & Acer rubrum & red maple & 1.3 & 0.20 \\
\hline T-57 RQP1 & LIST & Liquidambar styraciflua & sweetgum & 1.3 & 0.18 \\
\hline T-57 RQP2 & QULA & Quercus laurifolia & laurel oak & 4.4 & 0.09 \\
\hline T-57 RQP3 & LIST & Liquidambar styraciflua & sweetgum & 1.0 & 0,30 \\
\hline T-57 RQP4 & LIST & Liquidambar styraciflua & sweetgum & 3.4 & 0.36 \\
\hline T-58 LQP1 & $\mathrm{ACRU}$ & Acer rubrum & red maple & 1.3 & 0.18 \\
\hline T-58 LQP2 & ACRU & Acer rubrum & red maple & 3.1 & 0.07 \\
\hline T-58 LQP3 & LIST & Liquidambar styraciflua & sweetgum & 2.8 & 0.16 \\
\hline T-58 LQP4 & ACRU & Acer rubrum & red maple & 2.2 & 0.10 \\
\hline T-58 RQP1 & NYAQ & Nyssa aquatica & water tupelo & 9.0 & 0.30 \\
\hline T-58 RQP2 & LIST & Liquidambar styraciflua & sweetgum & 2.1 & 0.43 \\
\hline T-58 RQP3 & LIST & Liquidambar styraciflua & sweetgum & 1.5 & 0.13 \\
\hline T-58 RQP4 & ACRU & Acer rubrum & red maple & 1.5 & 0.45 \\
\hline T-59 LQP1 & LIST & Liquidambar styraciflua & sweetgum & 2.8 & 0.27 \\
\hline T-59 LQP2 & LIST & Liquidambar styraciflua & sweetgum & 4.0 & 0.40 \\
\hline T-59 LQP3 & $\mathrm{ACRU}$ & Acer rubrum & red maple & 2.0 & 0.60 \\
\hline T-59 LQP4 & LIST & Liquidambar styraciflua & sweetgum & 1.4 & 0.23 \\
\hline T-59 RQP1 & $\mathrm{ACRU}$ & Acer rubrum & red maple & 2.0 & 0.47 \\
\hline T-59 RQP2 & FRCA & Fraxinus caroliniana & Carolina (water) ash & 3.6 & 0.03 \\
\hline T-59 RQP3 & LIST & Liquidambar styraciflua & sweetgum & 1.4 & 0.09 \\
\hline T-59 RQP4 & NYSY & Nyssa sylvatica & sourgum & 4.7 & 0.11 \\
\hline T-60 LQP1 & ACRU & Acer rubrum & red maple & 1.1 & 0.29 \\
\hline T-60 LQP2 & NYAQ & Nyssa sylvatica & sourgum & 10.4 & 0.24 \\
\hline T-60 LQP3 & ACRU & Acer rubrum & red maple & 6.2 & 0.60 \\
\hline T-60 LQP4 & NYSY & Nyssa sylvatica & sourgum & 1.3 & 0.10 \\
\hline T-60 RQP1 & $\mathrm{ACRU}$ & Acer rubrum & red maple & 2.5 & 0.04 \\
\hline T-60 RQP2 & ACRU & Acer rubrum & red maple & 8.0 & 0.33 \\
\hline T-60 RQP3 & ACRU & Acer rubrum & red maple & 4.2 & 0.33 \\
\hline T-60 RQP4 & NYSY & Nyssa sylvatica & sourgum & 3.3 & 0.24 \\
\hline
\end{tabular}


Table 19. Riparian-zone trees, Cedar Creek below Myers Creek near Hopkins, S.C., USGS 02169670 (reach 12) [m, meter(s); T-n, transect number; LQPn, left quarterpoint, nth tree; RQPn, right quarterpoint, nth tree]

\begin{tabular}{|c|c|c|c|c|c|}
\hline $\begin{array}{c}\text { Tree } \\
\text { position }\end{array}$ & $\begin{array}{l}\text { Species } \\
\text { code }\end{array}$ & Scientific name & Common name & $\begin{array}{l}\text { Distance } \\
\text { from pivot } \\
\text { point }(m)\end{array}$ & $\begin{array}{l}\text { Trunk diameter at } \\
\text { breast height }(\mathrm{m})\end{array}$ \\
\hline T-61 LQP1 & FRCA & Fraxinus caroliniana & Carolina (water) ash & 1.4 & 0.03 \\
\hline T-61 LQP2 & ILOP & Ilex opaca & American holly & 1.3 & 0.04 \\
\hline T-61 LQP3 & ILOP & Ilex opaca & American holly & 1.9 & 0.03 \\
\hline T-61 LQP4 & FRCA & Fraxinus caroliniana & Carolina (water) ash & 2.0 & 0.06 \\
\hline T-61 RQP1 & LIST & Liquidambar styraciflua & sweetgum & 0.7 & 0.20 \\
\hline T-61 RQP2 & ILOP & Ilex opaca & American holly & 1.3 & 0.05 \\
\hline T-61 RQP3 & NYAQ & Nyssa aquatica & water tupelo & 4.2 & 0.57 \\
\hline T-61 RQP4 & LIST & Liquidambar styraciflua & sweetgum & 0.8 & 0.07 \\
\hline T-62 LQP1 & LIST & Liquidambar styraciflua & sweetgum & 0.9 & 0.36 \\
\hline T-62 LQP2 & PEBO & Persea borbonia & redbay & 4.8 & 0.14 \\
\hline T-62 LQP3 & PEBO & Persea borbonia & redbay & 4.1 & 0.05 \\
\hline T-62 LQP4 & ILOP & Ilex ораса & American holly & 0.4 & 0.03 \\
\hline T-62 RQP1 & CYRA & Cyrilla racemiflora & cyrilla & 2.2 & 0.04 \\
\hline T-62 RQP2 & LIST & Liquidambar styraciflua & sweetgum & 1.3 & 0.36 \\
\hline T-62 RQP3 & LIST & Liquidambar styraciflua & sweetgum & 1.0 & 0.05 \\
\hline T-62 RQP4 & FRCA & Fraxinus caroliniana & Carolina (water) ash & 0.6 & 0.04 \\
\hline T-63 LQP1 & LIST & Liquidambar styraciflua & sweetgum & 0.8 & 0.43 \\
\hline T-63 LQP2 & ACRU & Acer rubrum & red maple & 2.9 & 0.14 \\
\hline T-63 LQP3 & ULAM & Ulmus alata & winged elm & 2.8 & 0.05 \\
\hline T-63 LQP4 & ACRU & Acer rubrum & red maple & 1.0 & 0.13 \\
\hline T-63 RQP1 & LIST & Liquidambar styraciflua & sweetgum & 1.4 & 0.37 \\
\hline T-63 RQP2 & ILOP & Ilex ораса & American holly & 1.4 & 0.10 \\
\hline T-63 RQP3 & ACRU & Acer rubrum & red maple & 6.9 & 0.15 \\
\hline T-63 RQP4 & ACRU & Acer rubrum & red maple & 3.1 & 0.75 \\
\hline T-64 LQP1 & ILOP & Ilex ораса & American holly & 0.7 & 0.19 \\
\hline T-64 LQP2 & ACRU & Acer rubrum & red maple & 5.8 & 0.37 \\
\hline T-64 LQP3 & FRCA & Fraxinus caroliniana & Carolina (water) ash & 1.2 & 0.10 \\
\hline T-64 LQP4 & ACRU & Acer rubrum & red maple & 2.0 & 0.39 \\
\hline T-64 RQP1 & ILOP & Ilex opaca & American holly & 0.5 & 0.03 \\
\hline T-64 RQP2 & ACRU & Acer rubrum & red maple & 0.8 & 0.31 \\
\hline T-64 RQP3 & NYAQ & Nyssa aquatica & water tupelo & 2.4 & 0.23 \\
\hline T-64 RQP4 & PEBO & Persea borbonia & redbay & 2.1 & 0.03 \\
\hline T-65 LQP1 & ACRU & Acer rubrum & red maple & 1.2 & 0.29 \\
\hline T-65 LQP2 & ILOP & Ilex ораса & American holly & 4.4 & 0.18 \\
\hline T-65 LQP3 & ILOP & Ilex ораса & American holly & 3.8 & 0.06 \\
\hline T-65 LQP4 & LIST & Liquidambar styraciflua & sweetgum & 2.1 & 0.30 \\
\hline T-65 RQP1 & QULA & Quercus laurifolia & laurel oak & 1.8 & 0.39 \\
\hline T-65 RQP2 & ACRU & Acer rubrum & red maple & 2.3 & 0.06 \\
\hline T-65 RQP3 & ACRU & Acer rubrum & red maple & 1.6 & 0.04 \\
\hline T-65 RQP4 & ACRU & Acer rubrum & red maple & 0.2 & 0.04 \\
\hline T-66 LQP1 & PEBO & Persea borbonia & redbay & 1.6. & 0.12 \\
\hline T-66 LQP2 & ACRU & Acer rubrum & red maple & 3.7 & 0.13 \\
\hline T-66 LQP3 & ILOP & Ilex ораса & American holly & 2.7 & 0.03 \\
\hline T-66 LQP4 & LIST & Liquidambar styraciflua & sweetgum & 2.0 & 0.05 \\
\hline T-66 RQP1 & ACRU & Acer rubrum & red maple & 1.1 & 0.49 \\
\hline T-66 RQP2 & CYRA & Cyrilla racemiflora & cyrilla & 0.9 & 0.06 \\
\hline T-66 RQP3 & ILOP & Ilex ораса & American holly & 0.6 & 0.04 \\
\hline T-66 RQP4 & LIST & Liquidambar styraciflua & sweetgum & 1.0 & 0.27 \\
\hline
\end{tabular}


Table 20. Riparian-zone trees, Cedar Creek at Cedar Creek Hunt Club near Gadsden, S.C., USGS 02169672 (reach 13) [m, meter(s); T-n, transect number; LQPn, left quarterpoint, nth tree; RQPn, right quarterpoint, nth tree; NI, not identified; ---, no data]

\begin{tabular}{|c|c|c|c|c|c|}
\hline $\begin{array}{c}\text { Tree } \\
\text { position }\end{array}$ & $\begin{array}{l}\text { Species } \\
\text { code }\end{array}$ & Scientific name & Common name & $\begin{array}{l}\text { Distance } \\
\text { from pivot } \\
\text { point (m) }\end{array}$ & $\begin{array}{l}\text { Trunk diameter a } \\
\text { breast height }(\mathrm{m})\end{array}$ \\
\hline T-67 LQP1 & CACA & Carpinus caroliniana & ironwood & 2.7 & 0.11 \\
\hline T-67 LQP2 & QUMI & Quercus michauxii & basket oak & 1.5 & 0.53 \\
\hline T-67 LQP3 & CACA & Carpinus caroliniana & ironwood & 3.7 & 0.03 \\
\hline T-67 LQP4 & NYAQ & Nyssa aquatica & water tupelo & 2.3 & 0.04 \\
\hline T-67 RQP1 & NI & -- & tree & 0.9 & 0.03 \\
\hline T-67 RQP2 & ULAM & Ulmus americana & American elm & 5.0 & 0.29 \\
\hline T-67 RQP3 & ULAM & Ulmus americana & American elm & 4.0 & 0.05 \\
\hline T-67 RQP4 & QUNI & Quercus nigra & water oak & 1.3 & 0.07 \\
\hline T-68 LQP1 & NYAQ & Nyssa aquatica & water tupelo & 3.0 & 0.47 \\
\hline T-68 LQP2 & NYAQ & Nyssa aquatica & water tupelo & 5.0 & 0.89 \\
\hline T-68 LQP3 & NYAQ & Nyssa aquatica & water tupelo & 1.5 & 0.94 \\
\hline T-68 LQP4 & NYAQ & Nyssa aquatica & water tupelo & 1.8 & 0.03 \\
\hline T-68 RQP1 & NYAQ & Nyssa aquatica & water tupelo & 1.0 & 0.30 \\
\hline T-68 RQP2 & CACA & Carpinus caroliniana & ironwood & 4.2 & 0.30 \\
\hline T-68 RQP3 & QULA & Quercus laurifolia & laurel oak & 2.2 & 0.63 \\
\hline T-68 RQP4 & TADI & Taxodium distichum & baldcypress & 3.4 & 0.64 \\
\hline T-69 LQP1 & NYAQ & Nyssa aquatica & water tupelo & 1.2 & 0.52 \\
\hline T-69 LQP2 & ACRU & Acer rubrum & red maple & 2.6 & 0.12 \\
\hline T-69 LQP3 & NYAQ & Nyssa aquatica & water tupelo & 5.0 & 0.57 \\
\hline T-69 LQP4 & NYAQ & Nyssa aquatica & water tupelo & 1.9 & 0.03 \\
\hline T-69 RQP1 & ILOP & Ilex opaca & American holly & 2.7 & 0.06 \\
\hline T-69 RQP2 & QULA & Quercus laurifolia & laurel oak & 4.7 & 0.41 \\
\hline T-69 RQP3 & QULA & Quercus laurifolia & laurel oak & 1.7 & 0.35 \\
\hline T-69 RQP4 & QULA & Quercus laurifolia & laurel oak & 1.7 & 0.07 \\
\hline T-70 LQP1 & NYAQ & Nyssa aquatica & water tupelo & 7.0 & 0.67 \\
\hline $\mathrm{T}-70 \mathrm{LQP} 2$ & NYAQ & Nyssa aquatica & water tupelo & 1.8 & 0.11 \\
\hline T-70 LQP3 & NYAQ & Nyssa aquatica & water tupelo & 1.3 & 0.29 \\
\hline T-70 LQP4 & NYAQ & Nyssa aquatica & water tupelo & 1.3 & 0.67 \\
\hline T-70 RQP1 & ILOP & Ilex ораса & American holly & 5.5 & 0.08 \\
\hline $\mathrm{T}-70 \mathrm{RQP2}$ & QULA & Quercus laurifolia & laurel oak & 0.8 & 0.71 \\
\hline T-70 RQP3 & QULA & Quercus laurifolia & laurel oak & 1.4 & 0.15 \\
\hline T-70 RQP4 & QULA & Quercus laurifolia & laurel oak & 0.7 & 0.16 \\
\hline T-71 LQP1 & NYAQ & Nyssa aquatica & water tupelo & 4.2 & 0.21 \\
\hline T-71 LQP2 & NYAQ & Nyssa aquatica & water tupelo & 3.6 & 0.30 \\
\hline T-71 LQP3 & NYAQ & Nyssa aquatica & water tupelo & 1.2 & 0.37 \\
\hline T-71 LQP4 & QULA & Quercus laurifolia & laurel oak & 0.6 & 0.30 \\
\hline T-71 RQPI & NYAQ & Nyssa aquatica & water tupelo & 1.6 & 0.54 \\
\hline $\mathrm{T}-71 \mathrm{RQP2}$ & NYAQ & Nyssa aquatica & water tupelo & 1.1 & 0.03 \\
\hline T-71 RQP3 & NYAQ & Nyssa aquatica & water tupelo & 0.9 & 0.72 \\
\hline T-71 RQP4 & NYAQ & Nyssa aquatica & water tupelo & 0.8 & 0.06 \\
\hline T-72 LQP1 & NYAQ & Nyssa aquatica & water tupelo & 8.4 & 0.51 \\
\hline T-72 LQP2 & CACA & Carpinus caroliniana & ironwood & 1.7 & 0.12 \\
\hline T-72 LQP3 & $\mathrm{ACRU}$ & Acer rubrum & red maple & 2.9 & 0.03 \\
\hline $\mathrm{T}-72 \mathrm{LQP} 4$ & NYAQ & Nyssa aquatica & water tupelo & 2.4 & 0.81 \\
\hline $\mathrm{T}-72 \mathrm{RQP1}$ & NYAQ & Nyssa aquatica & water tupelo & 2.0 & 0.06 \\
\hline T-72 RQP2 & NYAQ & Nyssa aquatica & water tupelo & 3.0 & 0.88 \\
\hline T-72 RQP3 & ACRU & Acer rubrum & red maple & 3.5 & 0.08 \\
\hline T-72 RQP4 & TADI & Taxodium distichum & baldcypress & 4.0 & 0.10 \\
\hline
\end{tabular}


Table 21. Riparian-zone trees, Toms Creek near Gadsden, S.C., USGS 021696966 (reach 14) [m, meter(s); T-n, transect number; LQPn, left quarterpoint, nth tree; RQPn, right quarterpoint, nth tree]

\begin{tabular}{|c|c|c|c|c|c|}
\hline $\begin{array}{c}\text { Tree } \\
\text { position }\end{array}$ & $\begin{array}{l}\text { Species } \\
\text { code }\end{array}$ & Scientific name & Common name & $\begin{array}{l}\text { Distance } \\
\text { from pivot } \\
\text { point }(m)\end{array}$ & $\begin{array}{l}\text { Trunk diameter at } \\
\text { breast height }(\mathrm{m})\end{array}$ \\
\hline T-73 LQP1 & QULA & Quercus laurifolia & laurel oak & 1.1 & 0.26 \\
\hline T-73 LQP2 & QULA & Quercus laurifolia & laurel oak & 2.0 & 0.35 \\
\hline T-73 LQP3 & LIST & Liquidambar styraciflua & sweetgum & 2.3 & 0.09 \\
\hline T-73 LQP4 & ACRU & Acer rubrum & red maple & 1.6 & 0.22 \\
\hline T-73 RQP1 & ACRU & Acer rubrum & red maple & 1.0 & 0.19 \\
\hline T-73 RQP2 & ACRU & Acer rubrum & red maple & 1.6 & 0.24 \\
\hline T-73 RQP3 & QULA & Quercus laurifolia & laurel oak & 2.0 & 0.34 \\
\hline T-73 RQP4 & QULA & Quercus laurifolia & laurel oak & 1.5 & 0.06 \\
\hline T-74 LQP1 & ACRU & Acer rubrum & red maple & 1.5 & 0.12 \\
\hline T-74 LQP2 & LIST & Liquidambar styraciflua & sweetgum & 2.4 & 0.22 \\
\hline T-74 LQP3 & ACRU & Acer rubrum & red maple & 32.5 & 0.08 \\
\hline T-74 LQP4 & ACRU & Acer rubrum & red maple & 1.6 & 0.19 \\
\hline $\mathrm{T}-74 \mathrm{RQP1}$ & CACA & Carpinus carolina & ironwood & 0.7 & 0.05 \\
\hline $\mathrm{T}-74 \mathrm{RQP} 2$ & QULA & Quercus laurifolia & laurel oak & 0.9 & 0.07 \\
\hline T-74 RQP3 & ACRU & Acer rubrum & red maple & 1.7 & 0.07 \\
\hline $\mathrm{T}-74 \mathrm{RQP} 4$ & ACRU & Acer rubrum & red maple & 1.6 & 0.06 \\
\hline T-75 LQP1 & QUIN & Quercus incana & bluejack oak & 2.1 & 0.06 \\
\hline T-75 LQP2 & TADI & Taxodium distichum & baldcypress & 2.8 & 0.02 \\
\hline T-75 LQP3 & ACRU & Acer rubrum & red maple & 2.8 & 0.33 \\
\hline T-75 LQP4 & PLAQ & Planera aquatica & water elm & 2.0 & 0.19 \\
\hline $\mathrm{T}-75 \mathrm{RQP1}$ & ACRU & Acer rubrum & red maple & 1.1 & 0.13 \\
\hline $\mathrm{T}-75 \mathrm{RQP} 2$ & ACRU & Acer rubrum & red maple & 1.6 & 0.24 \\
\hline T-75 RQP3 & LIST & Liquidambar styraciflua & sweetgum & 3.6 & 0.21 \\
\hline T-75 RQP4 & QULA & Quercus laurifolia & laurel oak & 2.2 & 0.07 \\
\hline T-76 LQP1 & LIST & Liquidambar styraciflua & sweetgum & 1.7 & 0.20 \\
\hline T-76 LQP2 & LIST & Liquidambar styraciflua & sweetgum & 1.1 & 0.45 \\
\hline T-76 LQP3 & ACRU & Acer rubrum & red maple & 1.9 & 0.04 \\
\hline T-76 LQP4 & QUAL & Quercus alba & white oak & 1.6 & 0.43 \\
\hline T-76 RQP1 & ACRU & Acer rubrum & red maple & 2.6 & 0.14 \\
\hline T-76 RQP2 & ACRU & Acer rubrum & red maple & 1.2 & 0.12 \\
\hline T-76 RQP3 & ACRU & Acer rubrum & red maple & 1.8 & 0.22 \\
\hline T-76 RQP4 & LIST & Liquidambar styraciflua & sweetgum & 4.5 & 0.18 \\
\hline T-77 LQP1 & ILOP & Ilex ораса & American holly & 1.7 & 0.06 \\
\hline T-77 LQP2 & QULA & Quercus laurifolia & laurel oak & 1.5 & 0.35 \\
\hline T-77 LQP3 & LIST & Liquidambar styraciflua & sweetgum & 2.4 & 0.13 \\
\hline T-77 LQP4 & TADI & Taxodium distichum & baldcypress & 1.6 & 0.36 \\
\hline T-77 RQP1 & LIST & Liquidambar styraciflua & sweetgum & 1.4 & 0.33 \\
\hline T-77 RQP2 & QULA & Quercus laurifolia & laurel oak & 2.7 & 0.41 \\
\hline T-77 RQP3 & QULA & Quercus laurifolia & laurel oak & 1.5 & 0.31 \\
\hline T-77 RQP4 & QULA & Quercus laurifolia & laurel oak & 1.9 & 0.43 \\
\hline T-78 LQP1 & LIST & Liquidambar styraciflua & sweetgum & 1.2 & 0.33 \\
\hline T-78 LQP2 & QULA & Quercus laurifolia & laurel oak & 3.1 & 0.26 \\
\hline T-78 LQP3 & QULA & Quercus laurifolia & laurel oak & 1.4 & 0.44 \\
\hline T-78 LQP4 & ACRU & Acer rubrum & red maple & 2.9 & 0.38 \\
\hline T-78 RQP1 & PLAQ & Planera aquatica & water elm & 1.1 & 0.04 \\
\hline T-78 RQP2 & QULA & Quercus laurifolia & laurel oak & 4.4 & 0.43 \\
\hline T-78 RQP3 & ACRU & Acer rubrum & red maple & 4.6 & 0.10 \\
\hline T-78 RQP4 & LIST & Liquidambar styraciflua & sweetgum & 1.7 & 0.37 \\
\hline
\end{tabular}


Table 22. Riparian-zone trees, McTier Creek near Monetta, S.C., USGS 02172300 (reach 15)

[m, meter(s); T-n, transect number; LQPn, left quarterpoint, nth tree; RQPn, right quarterpoint, $n$th tree]

\begin{tabular}{|c|c|c|c|c|c|}
\hline $\begin{array}{c}\text { Tree } \\
\text { position }\end{array}$ & $\begin{array}{l}\text { Species } \\
\text { code }\end{array}$ & Scientific name & Common name & $\begin{array}{l}\text { Distance } \\
\text { from pivot } \\
\text { point (m) }\end{array}$ & $\begin{array}{l}\text { Trunk diameter at } \\
\text { breast height (m) }\end{array}$ \\
\hline T-79 LQP1 & LIST & Liquidambar styraciflua & sweetgum & 2.0 & 0.45 \\
\hline T-79 LQP2 & LIST & Liquidambar styraciflua & sweetgum & 1.5 & 0.13 \\
\hline T-79 LQP3 & LIST & Liquidambar styraciflua & sweetgum & 1.0 & 0.41 \\
\hline T-79 LQP4 & ACRU & Acer rubrum & red maple & 7.4 & 0.44 \\
\hline T-79 RQP1 & LIST & Liquidambar styraciflua & sweetgum & 2.5 & 0.23 \\
\hline T-79 RQP2 & LITU & Liriodendron tulipifera & tulip tree & 3.2 & 0.41 \\
\hline T-79 RQP3 & CAGL & Carya glabra & pignut hickory & 6.3 & 0.11 \\
\hline T-79 RQP4 & CAGL & Carya glabra & pignut hickory & 4.1 & 0.15 \\
\hline T-80 LQP1 & LITU & Liriodendron tulipifera & tulip tree & 2.7 & 0.73 \\
\hline T-80 LQP2 & LIST & Liquidambar styraciflua & sweetgum & 3.0 & 0.61 \\
\hline T-80 LQP3 & QULA & Quercus laurifolia & laurel oak & 2.8 & 0.34 \\
\hline T-80 LQP4 & LIST & Liquidambar styraciflua & sweetgum & 5.0 & 0.07 \\
\hline T-80 RQP1 & ILOP & Ilex opaca & American holly & 2.9 & 0.11 \\
\hline T-80 RQP2 & ACRU & Acer rubrum & red maple & 5.4 & 0.39 \\
\hline T-80 RQP3 & QULA & Quercus laurifolia & laurel oak & 6.2 & 0.24 \\
\hline T-80 RQP4 & LIST & Liquidambar styraciflua & sweetgum & 3.3 & 0.13 \\
\hline T-81 LQP1 & QULA & Quercus laurifolia & laurel oak & 3.9 & 0.43 \\
\hline T-81 LQP2 & LIST & Liquidambar styraciflua & sweetgum & 8.7 & 0.55 \\
\hline T-81 LQP3 & ILOP & Ilex opaca & American holly & 6.1 & 0.19 \\
\hline T-81 LQP4 & ACRU & Acer rubrum & red maple & 6.9 & 0.45 \\
\hline T-81 RQP1 & ACRU & Acer rubrum & red maple & 0.8 & 0.05 \\
\hline $\mathrm{T}-81 \mathrm{RQP} 2$ & ACRU & Acer rubrum & red maple & 1.3 & 0.09 \\
\hline T-81 RQP3 & ILOP & Ilex opaca & American holly & 2.2 & 0.07 \\
\hline T-81 RQP4 & ACRU & Acer rubrum & red maple & 0.8 & 0.06 \\
\hline T-82 LQP1 & LITU & Liriodendron tulipifera & tulip tree & 3.0 & 0.62 \\
\hline T-82 LQP2 & ILOP & Ilex ораса & American holly & 1.9 & 0.07 \\
\hline T-82 LQP3 & ILOP & Ilex opaca & American holly & 9.7 & 0.32 \\
\hline T-82 LQP4 & ACRU & Acer rubrum & red maple & 6.9 & 0.10 \\
\hline T-82 RQP1 & QUNI & Quercus nigra & water oak & 1.3 & 0.23 \\
\hline T-82 RQP2 & LIST & Liquidambar styraciflua & sweetgum & 0.6 & 0.16 \\
\hline T-82 RQP3 & ILOP & Ilex opaca & American holly & 2.8 & 0.1 \\
\hline T-82 RQP4 & ACRU & Acer rubrum & red maple & 4.3 & 0.10 \\
\hline T-83 LQP1 & QUNI & Quercus nigra & water oak & 3.2 & 0.34 \\
\hline T-83 LQP2 & QUNI & Quercus nigra & water oak & 2.7 & 0.43 \\
\hline T-83 LQP3 & QULA & Quercus laurifolia & laurel oak & 2.6 & 0.15 \\
\hline T-83 LQP4 & QULA & Quercus laurifolia & laurel oak & 4.8 & 0.16 \\
\hline T-83 RQP1 & LIST & Liquidambar styraciflua & sweetgum & 2.2 & 0.46 \\
\hline T-83 RQP2 & LITU & Liriodendron tulipifera & tulip tree & 3.5 & 0.10 \\
\hline T-83 RQP3 & ACRU & Acer rubrum & red maple & 6.5 & 0.36 \\
\hline T-83 RQP4 & ACRU & Acer rubrum & red maple & 3.4 & 0.38 \\
\hline T-84 LQP1 & QUNI & Quercus nigra & water oak & 1.9 & 0.41 \\
\hline T-84 LQP2 & QUST & Quercus stellata & post oak & 1.4 & 0.07 \\
\hline T-84 LQP3 & ACRU & Acer rubrum & red maple & 2.4 & 0.09 \\
\hline T-84 LQP4 & PEBO & Persea borbonia & red bay & 3.3 & 0.06 \\
\hline T-84 RQP1 & LIST & Liquidambar styraciflua & sweetgum & 1.0 & 0.41 \\
\hline T-84 RQP2 & LIST & Liquidambar styraciflua & sweetgum & 2.0 & 0.21 \\
\hline T-84 RQP3 & LIST & Liquidambar styraciflua & sweetgum & 4.9 & 0.27 \\
\hline T-84 RQP4 & ACRU & Acer rubrum & red maple & 3.0 & 0.12 \\
\hline
\end{tabular}


Table 23. Riparian-zone trees, Cow Castle Creek near Bowman, S.C., USGS 02174250 (reach 16)

[m, meter(s); T-n, transect number; LQPn, left quarterpoint, nth tree; ---, no data; RQPn, right quarterpoint, nth tree; NI, not identified]

\begin{tabular}{|c|c|c|c|c|c|}
\hline Tree position & Species code & Scientific name & Common name & $\begin{array}{l}\text { Distance } \\
\text { from pivot } \\
\text { point }(\mathrm{m})\end{array}$ & $\begin{array}{l}\text { Trunk diameter at } \\
\text { breast height }(\mathrm{m})\end{array}$ \\
\hline T-85 LQP1 & LIST & Liquidambar styraciflua & sweetgum & 2.0 & 0.34 \\
\hline T-85 LQP2 & QUNI & Quercus nigra & water oak & 2.5 & 0.32 \\
\hline T-85 LQP3 & CAPA & Carya pallida & sand hickory & 4.0 & 0.38 \\
\hline T-85 LQP4 & CASP & Carya sp. & hickory & --- & --- \\
\hline T-85 RQP1 & CASP & Carya sp. & hickory & --- & --- \\
\hline T-85 RQP2 & CASP & Carya sp. & hickory & -- & --- \\
\hline T-85 RQP3 & CASP & Carya sp. & hickory & -- & -- \\
\hline T-85 RQP4 & CASP & Carya sp. & hickory & -- & --- \\
\hline T-86 LQPI & PLAQ & Planera aquatica & water elm & 1.2 & 0.39 \\
\hline T-86 LQP2 & QUNI & Quercus nigra & water oak & 2.3 & 0.06 \\
\hline T-86 LQP3 & CASP & Carya sp. & hickory & --- & --- \\
\hline T-86 LQP4 & CASP & Carya sp. & hickory & -- & -- \\
\hline T-86 RQP1 & ACRU & Acer rubrum & red maple & 1.0 & 0.37 \\
\hline T-86 RQP2 & QULA & Quercus laurifolia & laurel oak & 0.2 & 0.09 \\
\hline T-86 RQP3 & QULA & Quercus laurifolia & laurel oak & 0.2 & 0.04 \\
\hline T-86 RQP4 & LIST & Liquidambar styraciflua & sweetgum & 1.9 & 0.29 \\
\hline T-87 LQP1 & PLAQ & Planera aquatica & water elm & 1.5 & 0.05 \\
\hline T-87 LQP2 & ACRU & Acer rubrum & red maple & 3.6 & 0.12 \\
\hline T-87 LQP3 & CACA & Carpinus caroliniana & ironwood & 3.2 & 0.01 \\
\hline T-87 LQP4 & QULA & Quercus laurifolia & laurel oak & 2.7 & 0.17 \\
\hline T-87 RQPI & LITU & Liriodendron tulipifera & tulip poplar & 1.4 & 0.10 \\
\hline T-87 RQP2 & QUMI & Quercus michauxii & basket oak & 0.9 & 0.16 \\
\hline T-87 RQP3 & LIST & Liquidambar styraciflua & sweetgum & 1.4 & 0.19 \\
\hline T-87 RQP4 & LIST & Liquidambar styraciflua & sweetgum & 2.0 & 0.03 \\
\hline T-88 LQP1 & QULA & Quercus laurifolia & laurel oak & 5.4 & 0.08 \\
\hline T-88 LQP2 & $\mathrm{CACA}$ & Carpinus caroliniana & ironwood & 2.3 & 0.11 \\
\hline T-88 LQP3 & QULA & Quercus laurifolia & laurel oak & 3.2 & 0.32 \\
\hline T-88 LQP4 & LIST & Liquidambar styraciflua & sweetgum & 2.7 & 0.36 \\
\hline T-88 RQP1 & QULA & Quercus laurifolia & laurel oak & 42 & 0.04 \\
\hline T-88 RQP2 & ACRU & Acer rubrum & red maple & 2.5 & 0.35 \\
\hline T-88 RQP3 & ACRU & Acer rubrum & red maple & 2.2 & 0.28 \\
\hline T-88 RQP4 & CACA & Carpinus caroliniana & ironwond & 2.4 & 0.16 \\
\hline T-89 LQPI & ULAM & Ulmus americana & slippery elm & 1.3 & 0.25 \\
\hline T-89 LQP2 & LIST & Liquidambar styraciflua & sweetgum & 2.5 & 0.47 \\
\hline T-89 LQP3 & NI & tree & -- & -- & -- \\
\hline T-89 LQP4 & NI & tree & -- & --- & -- \\
\hline T-89 RQP1 & QUMI & Quercus michauxii & basket oak & 1.3 & 0.18 \\
\hline T-89 RQP2 & COFL & Cornus florida & flowering dogwood & 2.9 & 0.11 \\
\hline T-89 RQP3 & ACRU & Acer rubrum & red maple & 3.5 & 0.16 \\
\hline T-89 RQP4 & QULA & Quercus laurifolia & laurel oak & 4.0 & 0.05 \\
\hline T-90 LQP1 & PLAQ & Planera aquatica & water elm & 0.2 & 0.23 \\
\hline T-90 LQP2 & QUNI & Quercus nigra & water oak & 0.2 & 0.14 \\
\hline T-90 LQP3 & QULA & Quercus laurifolia & laurel oak & 0.7 & 0.06 \\
\hline T-90 LQP4 & PLOX & Platanus occidentalis & sycamore & 6.8 & 0.28 \\
\hline T-90 RQPI & ACRU & Acer rubrum & red maple & 0.7 & 0.09 \\
\hline T-90 RQP2 & CACA & Carpinus caroliniana & ironwood & 1.7 & 0.19 \\
\hline T-90 RQP3 & PEBO & Persea borbonia & redbay & 3.3 & 0.03 \\
\hline T-90 RQP4 & QUN1 & Quercus nigra & water oak & 1.4 & 0.05 \\
\hline
\end{tabular}


Table 24. Riparian-zone trees, Cow Castle Creek near Bowman, S.C., USGS 02174250 (reach 17)

[m, meter(s); T-n, transect number; LQPn, left quarterpoint, nth tree; RQPn, right quarterpoint, nth tree]

\begin{tabular}{|c|c|c|c|c|c|}
\hline $\begin{array}{l}\text { Tree } \\
\text { position }\end{array}$ & $\begin{array}{l}\text { Species } \\
\text { code }\end{array}$ & Scientific name & Common name & $\begin{array}{c}\text { Distance } \\
\text { from pivot } \\
\text { point }(\mathrm{m})\end{array}$ & $\begin{array}{l}\text { Trunk diameter at } \\
\text { breast height }(\mathrm{m})\end{array}$ \\
\hline T-91 LQP1 & QUIN & Quercus incana & bluejack oak & 1.7 & 0.12 \\
\hline T-91 LQP2 & LIST & Liquidambar styraciflua & sweetgum & 3.2 & 0.17 \\
\hline T-91 LQP3 & LIST & Liquidambar styraciflua & sweetgum & 2.2 & 0.36 \\
\hline T-91 LQP4 & FRAM & Fraxinus americana & white ash & 2.4 & 0.10 \\
\hline T-91 RQP1 & QUMI & Quercus michauxii & basket oak & 2.1 & 0.57 \\
\hline T-91 RQP2 & CACA & Carpinus caroliniana & ironwood & 1.8 & 0.08 \\
\hline T-91 RQP3 & LIST & Liquidambar styraciflua & sweetgum & 2.9 & 0.10 \\
\hline T-91 RQP4 & CACA & Carpinus caroliniana & ironwood & 3.0 & 0.14 \\
\hline T-92 LQP1 & QURU & Quercus rubra & northern red oak & 2.9 & 0.20 \\
\hline T-92 LQP2 & LIST & Liquidambar styraciflua & sweetgum & 2.9 & 0.04 \\
\hline T-92 LQP3 & BRPA & Broussonetia papyrifera & paper mulberry & 2.0 & 0.09 \\
\hline T-92 LQP4 & CAGL & Carya glabra & pignut hickory & 3.0 & 0.10 \\
\hline T-92 RQP1 & QUNI & Quercus nigra & water oak & 2.8 & 0.09 \\
\hline T-92 RQP2 & $\mathrm{CACA}$ & Carpinus caroliniana & ironwood & 1.9 & 0.07 \\
\hline T-92 RQP3 & QULA & Quercus laurifolia & laurel oak & 2.1 & 0.09 \\
\hline T-92 RQP4 & CACA & Carpinus caroliniana & ironwood & 2.7 & 0.19 \\
\hline T-93 LQP1 & LITU & Liriodendron tulipifera & tulip poplar & 1.8 & 0.03 \\
\hline T-93 LQP2 & LIST & Liquidambar styraciflua & sweetgum & 3.5 & 0.34 \\
\hline T-93 LQP3 & PEBO & Persea borbonia & redbay & 1.8 & 0.05 \\
\hline T-93 LQP4 & LITU & Liriodendron tulipifera & tulip poplar & 5.3 & 0.52 \\
\hline T-93 RQP1 & ACRU & Acer rubrum & red maple & 1.8 & 0.21 \\
\hline T-93 RQP2 & LITU & Liriodendron tulipifera & tulip poplar & 1.0 & 0.09 \\
\hline T-93 RQP3 & QULA & Quercus laurifolia & ironwood & 1.7 & 0.03 \\
\hline T-93 RQP4 & ACRU & Acer rubrum & red maple & 2.7 & 0.05 \\
\hline T-94 LQP1 & QURU & Quercus rubra & northern red oak & 1.2 & 0.29 \\
\hline T-94 LQP2 & PITA & Pinus taeda & loblolly pine & 5.0 & 0.63 \\
\hline T-94 LQP3 & $\mathrm{CACA}$ & Carpinus caroliniana & ironwood & 1.3 & 0.10 \\
\hline T-94 LQP4 & QULA & Quercus laurifolia & laurel oak & 5.9 & 0.07 \\
\hline T-94 RQP1 & ACRU & Acer rubrum & red maple & 3.9 & 0.04 \\
\hline T-94 RQP2 & COFL & Cornus florida & flowering dogwood & 1.7 & 0.11 \\
\hline T-94 RQP3 & QUNI & Quercus nigra & water oak & 2.6 & 0.21 \\
\hline T-94 RQP4 & ACRU & Acer rubrum & red maple & 2.8 & 0.13 \\
\hline T-95 LQPI & ACRU & Acer rubrum & red maple & 3.2 & 0.58 \\
\hline T-95 LQP2 & PLAQ & Planera aquatica & water elm & 2.7 & 0.08 \\
\hline T-95 LQP3 & LIST & Liquidambar styraciflua & sweetgum & 2.9 & 0.37 \\
\hline T-95 LQP4 & LIST & Liquidambar styraciflua & sweetgum & 2.2 & 0.47 \\
\hline T-95 RQP1 & LIST & Liquidambar styraciflua & sweetgum & 4.8 & 0.24 \\
\hline T-95 RQP2 & QULA & Quercus laurifolia & laurel oak & 2.2 & 0.09 \\
\hline T-95 RQP3 & CACA & Carpinus caroliniana & ironwood & 0.7 & 0.15 \\
\hline T-95 RQP4 & LITU & Liriodendron tulipifera & tulip poplar & 1.1 & 0.25 \\
\hline T-96 LQP1 & QUMI & Quercus michauxii & basket oak & 1.6 & 0.31 \\
\hline T-96 LQP2 & LIST & Carpinus caroliniana & ironwood & 2.2 & 0.46 \\
\hline T-96 LQP3 & LIST & Liquidambar styraciflua & sweetgum & 4.7 & 0.51 \\
\hline T-96 LQP4 & QUMI & Quercus michauxii & basket oak & 4.5 & 0.12 \\
\hline T-96 RQP1 & CACA & Carpinus caroliniana & ironwood & 1.3 & 0.12 \\
\hline T-96 RQP2 & LIST & Liquidambar styraciflua & sweetgum & 0.6 & 0.3 \\
\hline T-96 RQP3 & LIST & Liquidambar styraciflua & sweetgum & 1.2 & 0.26 \\
\hline T-96 RQP4 & LIST & Liquidambar styraciflua & sweetgum & 4.1 & 0.21 \\
\hline
\end{tabular}


Table 25. Riparian-zone trees, Cow Castle Creek near Bowman, S.C., USGS 02174250 (reach 18)

[ $m$, meter(s); T-n, transect number; LQPn, left quarterpoint, $n$th tree; RQPn, right quarterpoint, $n$th tree]

\begin{tabular}{|c|c|c|c|c|c|}
\hline Tree position & Species code & Scientific name & Common name & $\begin{array}{l}\text { Distance } \\
\text { from pivot } \\
\text { point }(\mathrm{m})\end{array}$ & $\begin{array}{l}\text { Trunk diameter at } \\
\text { breast height }(\mathrm{m})\end{array}$ \\
\hline T-97 LQP1 & CAPA & Carya pallida & sand hickory & 1.1 & 0.08 \\
\hline T-97 LQP2 & QUNI & Quercus nigra & water oak & 1.6 & 0.38 \\
\hline T-97 LQP3 & CAVO & Carya ovata & shagbark hickory & 2.3 & 0.06 \\
\hline T-97 LQP4 & CAPA & Carpinus caroliniana & ironwood & 4.7 & 0.07 \\
\hline T-97 RQP1 & LIST & Liquidambar styraciflua & sweetgum & 3.4 & 0.25 \\
\hline T-97 RQP2 & CACA & Carpinus caroliniana & ironwood & 2 & 0.05 \\
\hline T-97 RQP3 & CAVO & Carya ovata & shagbark hickory & 5.3 & 0.15 \\
\hline T-97 RQP4 & LIST & Liquidambar styraciflua & sweetgum & 3.3 & 0.25 \\
\hline T-98 LQP1 & QUMI & Quercus michauxii & basket oak & 4.1 & 0.65 \\
\hline T-98 LQP2 & LIST & Liquidambar styraciflua & sweetgum & 3.6 & 0.07 \\
\hline T-98 LQP3 & LIST & Liquidambar styraciflua & sweetgum & 1.8 & 0.21 \\
\hline T-98 LQP4 & LIST & Liquidambar styraciflua & sweetgum & 1.1 & 0.06 \\
\hline T-98 RQP1 & QUNI & Quercus nigra & water oak & 1.6 & 0.10 \\
\hline T-98 RQP2 & LIST & Liquidambar styraciflua & sweetgum & 2.9 & 0.50 \\
\hline T-98 RQP3 & CAPA & Carpinus caroliniana & ironwood & 2 & 0.08 \\
\hline T-98 RQP4 & ACRU & Acer rubrum & red maple & 2.7 & 0.05 \\
\hline T-99 LQP1 & LIST & Liquidambar styraciflua & sweetgum & 2.9 & 0.17 \\
\hline T-99 LQP2 & CAGL & Carya glabra & pignut hickory & 1.6 & 0.04 \\
\hline T-99 LQP3 & QUMI & Quercus michauxii & basket oak & 2.3 & 0.23 \\
\hline T-99 LQP4 & QUMI & Quercus michauxii & basket oak & 1.5 & 0.05 \\
\hline T-99 RQP1 & QULA & Quercus laurifolia & laurel oak & 0.9 & 0.07 \\
\hline T-99 RQP2 & LIST & Liquidambar styraciflua & sweetgum & 0.5 & 0.09 \\
\hline T-99 RQP3 & CAOV & Carya ovata & shagbark hickory & 3.5 & 0.10 \\
\hline T-99 RQP4 & LITU & Liriodendron tulipifera & tulip poplar & 1.4 & 0.05 \\
\hline T-100 LQP1 & CAPA & Carya pallida & sand hickory & 1.8 & 0.17 \\
\hline T-100 LQP2 & LIST & Liquidambar styraciflua & sweetgum & 3.4 & 0.41 \\
\hline T-100 LQP3 & CAOV & Carya ovata & shagbark hickory & 1.7 & 0.05 \\
\hline T-100 LQP4 & ACRU & Acer rubrum & red maple & 4.1 & 0.12 \\
\hline T-100 RQP1 & LIST & Liquidambar styraciflua & sweetgum & 4.2 & 0.36 \\
\hline T-100 RQP2 & ACRU & Acer rubrum & red maple & 4 & 0.21 \\
\hline T-100 RQP3 & LIST & Liquidambar styraciflua & sweetgum & 1.9 & 0.16 \\
\hline T-100 RQP4 & LIST & Liquidambar styraciflua & sweetgum & 4.7 & 0.20 \\
\hline T-101 LQP1 & PIEC & Pinus echinata & shortleaf pine & 2.2 & 0.16 \\
\hline T-101 LQP2 & CAPA & Carya pallida & sand hickory & 2.8 & 0.17 \\
\hline T-101 LQP3 & LIST & Liquidambar styraciflua & sweetgum & 1 & 0.49 \\
\hline T-101 LQP4 & CAPA & Carya pallida & sand hickory & 3 & 0.09 \\
\hline T-101 RQP1 & ACRU & Acer rubrum & red maple & 1.8 & 0.28 \\
\hline T-101 RQP2 & COFL & Cornus florida & flowering dogwood & 3.5 & 0.10 \\
\hline T-101 RQP3 & CAPA & Carya pallida & sand hickory & 2.5 & 0.20 \\
\hline T-101 RQP4 & LIST & Liquidambar styraciflua & sweetgum & 0.7 & 0.28 \\
\hline T-102 LQP1 & LIST & Liquidambar styraciflua & sweetgum & 2.3 & 0.34 \\
\hline T-102 LQP2 & CAPA & Carya pallida & sand hickory & 1.5 & 0.06 \\
\hline T-102 LQP3 & QULY & Quercus lyrata & overcup oak & 1.5 & 0.06 \\
\hline T-102 LQP4 & CAOV & Carya ovata & shagbark hickory & 2.3 & 0.10 \\
\hline T-102 RQP1 & CAOV & Carya ovata & shagbark hickory & 2.3 & 0.11 \\
\hline T-102 RQP2 & CAOV & Carya ovata & shagbark hickory & 2.6 & 0.76 \\
\hline T-102 RQP3 & CAOV & Carya ovata & shagbark hickory & 1.8 & 0.35 \\
\hline T-102 RQP4 & CAOV & Carya ovata & shagbark hickory & 4.6 & 0.10 \\
\hline
\end{tabular}


Table 26. Riparian-zone trees, Coosawhatchie River near Grays, S.C., USGS 02176518 (reach 19)

[T-n, transect number; LQPn, left quarterpoint, $n$th tree; RQPn, right quarterpoint, $n$th tree]

\begin{tabular}{|c|c|c|c|c|c|}
\hline Tree position & Species code & Scientific name & Common name & $\begin{array}{l}\text { Distance } \\
\text { from pivot } \\
\text { point }(\mathrm{m})\end{array}$ & $\begin{array}{l}\text { Trunk diameter at } \\
\text { breast height }(\mathrm{m})\end{array}$ \\
\hline T-103 LQP1 & NYAQ & Nyssa aquatica & water tupelo & 1.5 & 0.51 \\
\hline T-103 LQP2 & FOAC & Forestiera acuminata & swamp forestiera & 2.7 & 0.13 \\
\hline T-103 LQP3 & NYAQ & Nyssa aquatica & water tupelo & 2 & 0.15 \\
\hline T-103 LQP4 & NYAQ & Nyssa aquatica & water tupelo & 2 & 0.19 \\
\hline T-103 RQP1 & ACRU & Acer rubrum & red maple & 0.8 & 0.10 \\
\hline T-103 RQP2 & NYAQ & Nyssa aquatica & water tupelo & 3.6 & 0.25 \\
\hline T-103 RQP3 & ULSP & Ulmaceae & elm & 2 & 0.53 \\
\hline T-103 RQP4 & LIST & Liquidambar styraciflua & sweetgum & 2.5 & 0.26 \\
\hline T-104 LQP1 & NYAQ & Nyssa aquatica & water tupelo & 3.5 & 0.68 \\
\hline T-104 LQP2 & CAAQ & Carya aquatica & water hickory & 1.2 & 0.09 \\
\hline T-104 LQP3 & ULSP & Ulmaceae & elm & 1.1 & 0.08 \\
\hline T-104 LQP4 & ULSP & Ulmaceae & elm & 2.1 & 0.03 \\
\hline T-104 RQP1 & QULA & Quercus laurifolia & laurel oak & 1.3 & 0.55 \\
\hline T-104 RQP2 & QUAL & Quercus lyrata & overcup oak & 2.1 & 0.24 \\
\hline T-104 RQP3 & TADI & Taxodium distichum & cypress & 1.7 & 0.44 \\
\hline T-104 RQP4 & FRCA & Fraxinus caroliniana & Carolina ash & 2.4 & 0.09 \\
\hline T-105 LQP1 & ULSP & Ulmaceae & elm & 1.2 & 0.30 \\
\hline T-105 LQP2 & QULA & Quercus laurifolia & laurel oak & 2.2 & 0.54 \\
\hline T-105 LQP3 & ULSP & Ulmaceae & elm & 1.6 & 0.04 \\
\hline T-105 LQP4 & QULA & Quercus laurifolia & laurel oak & 4.1 & 0.64 \\
\hline T-105 RQP1 & NYAQ & Nyssa aquatica & water tupelo & 2.8 & 0.32 \\
\hline T-105 RQP2 & LIST & Liquidambar styraciflua & sweetgum & 1.7 & 0.74 \\
\hline T-105 RQP3 & NYAQ & Nyssa aquatica & water tupelo & 1.2 & 0.16 \\
\hline T-105 RQP4 & ULSP & Ulmaceae & elm & 2.8 & 0.07 \\
\hline T-106 LQP1 & QULA & Quercus laurifolia & laurel oak & 1 & 0.24 \\
\hline T-106 LQP2 & LIST & Liquidambar styraciflua & sweetgum & 2 & 0.07 \\
\hline T-106 LQP3 & ACRU & Acer rubrum & red maple & 1.2 & 0.07 \\
\hline T-106 LQP4 & ULAQ & Ulmus aquatica & water elm & 1.2 & 0.34 \\
\hline T-106 RQP1 & NYAQ & Nyssa aquatica & water tupelo & 2.4 & 0.20 \\
\hline T-106 RQP2 & LIST & Liquidambar styraciflua & sweetgum & 6 & 0.17 \\
\hline T-106 RQP3 & ULSP & Ulmaceae & elm & 1.3 & 0.19 \\
\hline T-106 RQP4 & NYAQ & Nyssa aquatica & water tupelo & 1.1 & 0.43 \\
\hline T-107 LQP1 & ULSP & Ulmaceae & elm & 2.5 & 0.08 \\
\hline T-107 LQP2 & FRCA & Fraxinus caroliniana & Carolina ash & 2.3 & 0.11 \\
\hline T-107 LQP3 & NYAQ & Nyssa aquatica & water tupelo & 1.4 & 0.51 \\
\hline T-107 LQP4 & NYAQ & Nyssa aquatica & water tupelo & 5.1 & 0.57 \\
\hline T-107 RQP1 & LIST & Liquidambar styraciflua & sweetgum & 1 & 0.30 \\
\hline T-107 RQP2 & QUAL & Quercus lyrata & overcup oak & 2.1 & 0.10 \\
\hline T-107 RQP3 & $\mathrm{CACA}$ & Carpinus caroliniana & ironwood & 2.7 & 0.10 \\
\hline T-107 RQP4 & CAAQ & Carya aquatica & water hickory & 1.9 & 0.32 \\
\hline T-108 LQP1 & NYAQ & Nyssa aquatica & water tupelo & 1.2 & 0.13 \\
\hline T-108 LQP2 & NYAQ & Nyssa aquatica & water tupelo & 2.2 & 0.08 \\
\hline T-108 LQP3 & NYAQ & Nyssa aquatica & water tupelo & 0.9 & 0.29 \\
\hline T-108 LQP4 & FRCA & Fraxinus caroliniana & Carolina ash & 0.9 & 0.08 \\
\hline T-108 RQP1 & QUNI & Quercus nigra & water oak & 2.8 & 0.58 \\
\hline T-108 RQP2 & QUNI & Quercus nigra & water oak & 1.1 & 0.53 \\
\hline T-108 RQP3 & ULSP & Ulmaceae & elm & 4.3 & 0.09 \\
\hline T-108 RQP4 & CACA & Carpinus caroliniana & ironwood & 2 & 0.09 \\
\hline
\end{tabular}




\section{Algal Data}

Tables 27 and 28 list algal species and cell densities, respectively, determined from collections at in the Santee River Basin and Coastal Drainages study unit. Specific names and maximum relative abundance are reported. Unknown taxa are uniquely identified. A question mark (?) in the name (for example, Navicula sp.?) designates rare taxa that could be identified only to genus, or taxa for which the identification is tentative, but reasonable. Taxa that are almost, but not quite like a known taxon are designated with a "cf" (compare with). For clarity, the authorities for variety and form have been omitted from this listing. Interested readers should contact the Chief of the Santee River Basin and Coastal Drainages study unit for a more detailed listing of the algal species, including authority and miscellaneous information.

Taxonomic analysis was performed under contract by the Academy of Natural Sciences,

Philadelphia, $\mathrm{Pa}$. 
Table 27. Algal taxa and their maximum relative abundance in quantitative samples collected in the Santee River Basin and Coastal Drainages study unit

[Maximum relative abundance is the highest percentage of the total number of algal cells found in the sample set, and indicates the relative dominance of the taxon within the sample set. Collections of algal species include all quantitative and qualitative collections in the study unit. Blue-green algae, Cyanobacterium; ---, abundance too low to record]

\section{Algal group}

Algal taxon name

Blue-green algae

Blue-green algae

Blue-green algae

Blue-green algae

Blue-green algae

Blue-green algae

Blue-green algae

Blue-green algae

Blue-green algae

Blue-green algae

Blue-green algae

Blue-green algae

Blue-green algae

Blue-green algae

Blue-green algae

Blue-green algae

Blue-green algae

Blue-green algae

Blue-green algae

Blue-green algae

Blue-green algae

Cryptophytes

Diatoms

Diatoms

Diatoms

Diatoms

Diatoms

Diatoms

Diatoms

Diatoms

Diatoms

Diatoms

Diatoms

Diatoms

Diatoms

Diatoms

Diatoms

Diatoms

Diatoms

Diatoms

Diatoms

Diatoms

Diatoms

Diatoms

Diatoms

Diatoms

Diatoms

Diatoms

Diatoms
Anabaena affinis

Anabaena oscillarioides

Anabaena sp.

Calothrix parientina

Calothrix sp.

Hydrocoleum brebissonii

Hydrocoleum sp.

Lyngbya aestuarii

Lyngbya contorta

Lyngbya limnetica

Lyngbya martensiana

Lyngbya sp.

Merismopedia glauca

Microcoleus vaginatus

Microcystis sp.

Oscillatoria lutea

Oscillatoria princeps

Oscillatoria retzii

Oscillatoria sp.

Oscillatoria splendida

Spirulina laxa

Cryptomonas sp.

undifferentiated diatoms

Achnanthes bioreti

Achnanthes cf. grimmei

Achnanthes cf. lanceolata var. apiculata.

Achnanthes chlidanos

Achnanthes clevei

Achnanthes clevei var. rostrata

Achnanthes deflexa

Achnanthes delicatula.

Achnanthes exigua

Achnanthes exigua var, elliptica

Achnanthes exigua var. heterovalva

Achnanthes helvetica

Achnanthes hungarica

Achnanthes kryophila var. denistriata

Achnanthes lanceolata

Achnanthes lanceolata $\mathrm{cf}$. var. capitata

Achnanthes lanceolata var. abbreviata

Achnanthes lanceolata var. apiculata

Achnanthes lanceolata var. dubia

Achnanthes lanceolata var. haynaldii

Achnanthes lanceolata var. omissa

Achnanthes linearis

Achnanthes linearis

Achnanthes marginulata

Achnanthes microcephala

Achnanthes minutissima

\section{Number of samples in which organism was identified}

\section{Maximum relative abundance (percent)}

1.7

3.0

0.33

1.3

2.3

7.0

11.0

12

6.4

25

0.99

15

1

4.2

16

0.97

64

0.96

26.3

90.6

0.3

0.3

0.3

0.3

8.6

0.3

38.3

1.7

2.55

0.3

22.5

20.3

4.8

0.32

15.8

12.8

0.3

10.3

13.6

0.34

0.33

0.33

1.11

2.66

0.3

35.3 
Table 27. Algal taxa and their maximum relative abundance in quantitative samples collected in the Santee River Basin and Coastal Drainages study unit (Continued)

[Maximum relative abundance is the highest percentage of the total number of algal cells found in the sample set, and indicates the relative dominance of the taxon within the sample set. Collections of algal species include all quantitative and qualitative collections in the study unit. Blue-green algae, Cyanobacterium; ---, abundance too low to record]

\section{Algal group}

Algal taxon name

\section{Number of samples in which organism was identified}

\section{Maximum relative abundance (percent)}

Diatoms

Achnanthes peragalli

7

4.11

Diatoms

Achnanthes peragalli var. parvula

Achnanthes pinnata

Diatoms

Diatoms

Achnanthes rupestoides

Achnanthes semiaperta

Achnanthes sp.

Diatoms

Achnanthes subatomoides

Achnanthes subatomus

Diatoms

Diatoms

Diatoms

Diatoms

Diatoms

Diatoms

Diatoms

Diatoms

Diatoms

Diatoms

Diatoms

Diatoms

Diatoms

Diatoms

Diatoms

Diatoms

Diatoms

Diatoms

Diatoms

Diatoms

Diatoms

Diatoms

Diatoms

Diatoms

Diatoms

Diatoms

Diatoms

Diatoms

Diatoms

Diatoms

Diatoms

Diatoms

Diatoms

Diatoms

Diatoms

Diatoms

Diatoms

Diatoms

Diatoms

Diatoms

Diatoms

Diatoms

Diatoms
Achnanthes subhudsonis

Actinella punctata

Amphipleura pellucida

Amphora acutiuscula

Amphora cf. laevis

Amphora ovalis

Amphora ovalis var. affinis

Amphora ovalis var. pediculus

Amphora perpusilla

Amphora sp.

Amphora submontana

Amphora veneta

Anomoeoneis serians var. brachysira

Anomoeoneis vitrea

Aulacosira ambigua

Aulacosira crassipunctata

Aulacosira distans

Aulacosira distans var. nivalis

Aulacosira granulata

Aulacosira granulata var. angustissima

Aulacosira italica

Aulacosira lirata

Aulacosira lirata var. lacustris

Bacillaria paradoxa

Biddulphia laevis

Caloneis bacillum

Caloneis bacillum var. fontinalis

Caloneis hyalina

Caloneis limosa

Caloneis sp.?

Caloneis sp.

Caloneis ventricosa var. truncatula

Capartogramma crucicula

Cocconeis cf. diminuta

Cocconeis fluviatilis

Cocconeis neodiminuta

Cocconeis placentula var. euglypta

Cocconeis placentula var. lineata

Cocconeis sp.

Cyclotella atomus

Cyclotella meneghiniana

Cyclotella pseudostelligera
0.97

0.33

0.16

0.32

5.89

9.39

0.28

1.5

0.33

0.34

0.33

0.32

0.32

7.9

3.6

1.32

0.32

0.66

0.33

27.1

8.7

11

2.2

0.83

1.5

5.83

39

1.83

2.24

1.12

8.66

0.33

0.5

0.32

0.83

0.5

0.68

0.32

0.34

4.83

0.83

1.16

0.32

10.6

0.15

0.33

2

2.44 
Table 27. Algal taxa and their maximum relative abundance in quantitative samples collected in the Santee River Basin and Coastal Drainages study unit (Continued)

[Maximum relative abundance is the highest percentage of the total number of algal cells found in the sample set, and indicates the relative dominance of the taxon within the sample set. Collections of algal species include all quantitative and qualitative collections in the study unit. Blue-green algae, Cyanobacterium; ---, abundance too low to record]

Algal group

Algal taxon name

\section{Number of samples \\ in which organism was identified}

\section{Maximum relative abundance (percent)}

\begin{tabular}{|c|c|}
\hline Diatoms & Cyclotella stelligera \\
\hline Diatoms & Cymbella aspera \\
\hline Diatoms & Cymbella cuspidata \\
\hline Diatoms & Cymbella lunata \\
\hline Diatoms & Cymbella microcephala \\
\hline Diatoms & Cymbella minuta \\
\hline Diatoms & Cymbella minuta var. pseudogracilis \\
\hline Diatoms & Cymbella minuta var. silesiaca \\
\hline Diatoms & Cymbella naviculiformis \\
\hline Diatoms & Cymbella sp. \\
\hline Diatoms & Cymbella triangulum \\
\hline Diatoms & Cymbella tumida \\
\hline Diatoms & Cymbella tumidula \\
\hline Diatoms & Cymbella turgidula \\
\hline Diatoms & Desmogonium sp. \\
\hline Diatoms & Diploneis cf. ovalis \\
\hline Diatoms & Diploneis marginestriata \\
\hline Diatoms & Diploneis pseudovalis \\
\hline Diatoms & Diploneis puella \\
\hline Diatoms & Diploneis smithii \\
\hline Diatoms & Diploneis smithii var. pumila \\
\hline Diatoms & Entomoneis ornata \\
\hline Diatoms & Epithemia turgida \\
\hline Diatoms & Eunotia arcus \\
\hline Diatoms & Eunotia bidentula \\
\hline Diatoms & Eunotia camelus \\
\hline Diatoms & Eunotia carolina \\
\hline Diatoms & Eunotia cf. fallax var. gracillima \\
\hline Diatoms & Eunotia cf. formica \\
\hline Diatoms & Eunotia cf. tecta \\
\hline Diatoms & Eunotia cf. tenella \\
\hline Diatoms & Eunotia curvata \\
\hline Diatoms & Eunotia curvata var. subarcuata \\
\hline Diatoms & Eunotia elegans \\
\hline Diatoms & Eunotia exigua \\
\hline Diatoms & Eunotia faba \\
\hline Diatoms & Eunotia fallax var. gracillima \\
\hline Diatoms & Eunotia flexuosa \\
\hline Diatoms & Eunotia formica \\
\hline Diatoms & Eunotia hemicyclus \\
\hline Diatoms & Eunotia iatriaensis \\
\hline Diatoms & Eunotia incisa \\
\hline Diatoms & Eunotia intermedia \\
\hline Diatoms & Eunotia meisteri \\
\hline Diatoms & Eunotia microcephala \\
\hline Diatoms & Eunotia monodon \\
\hline Diatoms & Eunotia monodon var. major \\
\hline Diatoms & Eunotia naegelii \\
\hline Diatoms & Eunotia paludosa \\
\hline Diatoms & Eunotia pectinalis \\
\hline
\end{tabular}

1

4.32

7.33

0.33

7.83

0.95

12.5

0.98

8

1.14

0.34

0.3

8.66

0.47

0.66

0.86

0.33

0.32

0.66

0.97

0.33

3.11

0.16

0.16

0.32

1.7

0.16

6.16

0.32

0.34

0.5

0.15

1.33

16.2

0.33

0.66

0.3

0.16

7.33

9.5

0.16

1.5

3.83

0.79

7.66

2

0.32

0.45

5

11 
Table 27. Algal taxa and their maximum relative abundance in quantitative samples collected in the Santee River Basin and Coastal Drainages study unit (Continued)

[Maximum relative abundance is the highest percentage of the total number of algal cells found in the sample set, and indicates the relative dominance of the taxon within the sample set. Collections of algal species include all quantitative and qualitative collections in the study unit. Blue-green algae, Cyanobacterium; ---, abundance too low to record]

\section{Algal group}

\section{Algal taxon name}

\section{Number of samples in which organism was identified}

\section{Maximum relative abundance (percent)}

Diatoms

Diatoms

Diatoms

Diatoms

Diatoms

Diatoms

Diatoms

Diatoms

Diatoms

Diatoms

Diatoms

Diatoms

Diatoms

Diatoms

Diatoms

Diatoms

Diatoms

Diatoms

Diatoms

Diatoms

Diatoms

Diatoms

Diatoms

Diatoms

Diatoms

Diatoms

Diatoms

Diatoms

Diatoms

Diatoms

Diatoms

Diatoms

Diatoms

Diatoms

Diatoms

Diatoms

Diatoms

Diatoms

Diatoms

Diatoms

Diatoms

Diatoms

Diatoms

Diatoms

Diatoms

Diatoms

Diatoms

Diatoms

Diatoms

Diatoms
Eunotia pectinalis var. minor

Eunotia pectinalis var. undulata

Eunotia perpusilla

Eunotia polydentula

Eunotia praerupta

Eunotia rhomboidea

Eunotia robusta

Eunotia robusta var. tetraodon

Eunotia serra

Eunotia sp.

Eunotia sudetica

Eunotia tenella

Eunotia zasuminensis

Fragilaria bicapitata

Fragilaria brevistriata

Fragilaria capucina

Fragilaria capucina var. lanceolata

Fragilaria cf. capucina var. lanceolata

Fragilaria constricta

Fragilaria constricta var. trinodis

Fragilaria construens var. pumila

Fragilaria construens var. venter

Fragilaria crotonensis

Fragilaria gracillma

Fragilaria hungarica var. tumida

Fragilaria leptostauron

Fragilaria leptostauron var. dubia

Fragilaria nanana

Fragilaria pinnata

Fragilaria pinnata var. intercedens

Fragilaria sp.

Fragilaria tenera

Fragilaria vaucheriae

Fragilaria vaucheriae var. capitellata

Fragilaria virescens var. exigua

Frustulia rhomboides

Frustulia rhomboides form. undulata

Frustulia rhomboides var. capitata

Frustulia rhomboides var. crassinervia

Frustulia rhomboides var. saxonica

Frustulia sp.

Frustulia vulgaris

Frustulia weinholdii

Gomphonema affine

Gomphonema angustatum

Gomphonema angustatum var. obtusatum

Gomphonema angustatum var. productum

Gomphonema carolinense

Gomphonema cf. puiggarianum var. reducta

Gomphonema clevei
24

2

18

3

2

7

2

9

3

2

1

3

1

1

6

1

2

2

14

3

19

1

4

22

15

1

15

15

5
22.8

18.7

0.71

0.33

24.2

3.9

0.32

0.33

3.36

I. 33

1.33

23.3

0.5

2.5

2.97

1.58

2.06

0.15

0.33

1.66

0.66

1.66

0.32

0.33

0.16

0.32

13.2

1.00

0.16

3.16

1.66

4.81

0.96

0.33

10.5

2.97

4.66

11.3

36.3

0.16

1.98

2.46

0.45

3.83

0.33

3.69

0.31

0.32

37.0 
Table 27. Algal taxa and their maximum relative abundance in quantitative samples collected in the Santee River Basin and Coastal Drainages study unit (Continued)

[Maximum relative abundance is the highest percentage of the total number of algal cells found in the sample set, and indicates the relative dominance of the taxon within the sample set. Collections of algal species include all quantitative and qualitative collections in the study unit. Blue-green algae, Cyanobacterium; ---, abundance too low to record]

\section{Algal group}

Diatoms

Diatoms

Diatoms

Diatoms

Diatoms

Diatoms

Diatoms

Diatoms

Diatoms

Diatoms

Diatoms

Diatoms

Diatoms

Diatoms

Diatoms

Diatoms

Diatoms

Diatoms

Diatoms

Diatoms

Diatoms

Diatoms

Diatoms

Diatoms

Diatoms

Diatoms

Diatoms

Diatoms

Diatoms

Diatoms

Diatoms

Diatoms

Diatoms

Diatoms

Diatoms

Diatoms

Diatoms

Diatoms

Diatoms

Diatoms

Diatoms

Diatoms

Diatoms

Diatoms

Diatoms

Diatoms

Diatoms

Diatoms

Diatoms

Diatoms
Algal taxon name

\section{Maximum relative abundance (percent)}


Table 27. Algal taxa and their maximum relative abundance in quantitative samples collected in the Santee River Basin and Coastal Drainages study unit (Continued)

[Maximum relative abundance is the highest percentage of the total number of algal cells found in the sample set, and indicates the relative dominance of the taxon within the sample set. Collections of algal species include all quantitative and qualitative collections in the study unit. Blue-green algae, Cyanobacterium; ---, abundance too low to record]

\section{Algal group Algal taxon name}

\section{Number of samples in which organism was identified}

\section{Maximum relative abundance (percent)}

\begin{tabular}{|c|c|c|c|}
\hline Diatoms & Navicula cf. obsoleta & 3 & 0.66 \\
\hline Diatoms & Navicula cf. perparva & 2 & 1.36 \\
\hline Diatoms & Navicula cf. protracta & 1 & 1.63 \\
\hline Diatoms & Navicula cf. pseudoreinhardtii & 1 & 0.6 \\
\hline Diatoms & Navicula cf. saxophila & 1 & 0.3 \\
\hline Diatoms & Navicula cf. subarvensis & 1 & 2.16 \\
\hline Diatoms & Navicula cf. subarvensis & I & 1.63 \\
\hline Diatoms & Navicula cf. subrhynchocephala & 1 & 1.05 \\
\hline Diatoms & Navicula cf. subrotundata & 1 & 0.64 \\
\hline Diatoms & Navicula cf. subtilissima var. okamurae & 2 & 0.68 \\
\hline Diatoms & Navicula cf. trivialis & 1 & 1.44 \\
\hline Diatoms & Navicula cf. utermöhii & 2 & 5.52 \\
\hline Diatoms & Navicula cf. ventosa & 1 & 0.96 \\
\hline Diatoms & Navicula cf. zanoni & 1 & 2.13 \\
\hline Diatoms & Navicula clementis & 2 & 0.33 \\
\hline Diatoms & Navicula cocconeiformis & 3 & 1.13 \\
\hline Diatoms & Navicula cohnii & 8 & 0.61 \\
\hline Diatoms & Navicula confervacea & 9 & 5 \\
\hline Diatoms & Navicula confervacea form. rostrata & 1 & 0.26 \\
\hline Diatoms & Navicula contenta form. parallela & 1 & 0.97 \\
\hline Diatoms & Navicula contenta & 2 & 0.32 \\
\hline Diatoms & Navicula contenta var. biceps & 20 & 3.83 \\
\hline Diatoms & Navicula crucicula & 5 & 5.54 \\
\hline Diatoms & Navicula cryptocephala & 35 & 8.88 \\
\hline Diatoms & Navicula cryptocephala var. veneta & 10 & 0.66 \\
\hline Diatoms & Navicula cryptotenella & 10 & 4.66 \\
\hline Diatoms & Navicula decussis & 24 & 18.3 \\
\hline Diatoms & Navicula difficillima & 2 & 1.28 \\
\hline Diatoms & Navicula elginensis & 3 & 1 \\
\hline Diatoms & Navicula elginensis var. neglecta & 1 & 0.33 \\
\hline Diatoms & Navicula evanida & 3 & 0.16 \\
\hline Diatoms & Navicula exigua & 1 & 0.32 \\
\hline Diatoms & Navicula exigua var. capitata & 5 & 0.68 \\
\hline Diatoms & Navicula falaisensis var. lanceola & 1 & 0.66 \\
\hline Diatoms & Navicula festiva & 4 & 0.33 \\
\hline Diatoms & Navicula gallica var. laevissima & 1 & 0.33 \\
\hline Diatoms & Navicula gallica var. perpusilla & 2 & 0.33 \\
\hline Diatoms & Navicula gastrum & 1 & 0.16 \\
\hline Diatoms & Navicula goeppertiana & 2 & 0.66 \\
\hline Diatoms & Navicula graciloides & 2 & 1.16 \\
\hline Diatoms & Navicula gregaria & 12 & 10.8 \\
\hline Diatoms & Navicula halophila & 1 & 0.16 \\
\hline Diatoms & Navicula halophila form. tenuirostris & 1 & 0.31 \\
\hline Diatoms & Navicula hambergï & 3 & 0.33 \\
\hline Diatoms & Navicula hassiaca & 8 & 2.33 \\
\hline Diatoms & Navicula heufleri var. leptocephala & 4 & 0.33 \\
\hline Diatoms & Navicula hustedtii & 5 & 1.66 \\
\hline Diatoms & Navicula ignota var. acceptata & 1 & 0.5 \\
\hline Diatoms & Navicula indifferens & 1 & 0.14 \\
\hline Diatoms & Navicula ingenua & 1 & 0.16 \\
\hline
\end{tabular}


Table 27. Algal taxa and their maximum relative abundance in quantitative samples collected in the Santee River Basin and Coastal Drainages study unit (Continued)

[Maximum relative abundance is the highest percentage of the total number of algal cells found in the sample set, and indicates the relative dominance of the taxon within the sample set. Collections of algal species include all quantitative and qualitative collections in the study unit. Blue-green algae, Cyanobacterium; ---, abundance too low to record]

\section{Algal group}

\section{Algal taxon name}

\section{Number of samples in which organism was identified}

\section{Maximum relative abundance (percent)}

Diatoms

Diatoms

Diatoms

Diatoms

Diatoms

Diatoms

Diatoms

Diatoms

Diatoms

Diatoms

Diatoms

Diatoms

Diatoms

Diatoms

Diatoms

Diatoms

Diatoms

Diatoms

Diatoms

Diatoms

Diatoms

Diatoms

Diatoms

Diatoms

Diatoms

Diatoms

Diatoms

Diatoms

Diatoms

Diatoms

Diatoms

Diatoms

Diatoms

Diatoms

Diatoms

Diatoms

Diatoms

Diatoms

Diatoms

Diatoms

Diatoms

Diatoms

Diatoms

Diatoms

Diatoms

Diatoms

Diatoms

Diatoms

Diatoms

Diatoms
Navicula invicta

Navicula kotschyi

Navicula krasskei

Navicula laevissima

Navicula lanceolata

Navicula lateropunctata

Navicula leptostriata

Navicula luzonensis

Navicula mediacomplexa

Navicula menisculus var. upsaliensis

Navicula minima

Navicula minuscula

Navicula minuscula var. muralis

Navicula mitigata

Navicula mobiliensis var. minor

Navicula modica

Navicula molestiformis

Navicula mutica form. undulata

Navicula mutica

Navicula mutica var. stigma

Navicula mutica var. ventricosa

Navicula obsidialis

Navicula omissa

Navicula pelliculosa

Navicula perminuta

Navicula placentula

Navicula pseudoreinhardtii

Navicula pseudoventralis

Navicula pupula

Navicula pupula var. capitata

Navicula pupula var. mutata

Navicula pupula var. rectangularis

Navicula pupula var. rostrata

Navicula pusilla.

Navicula radiosa

Navicula rhynchocephala

Navicula rhynchocephala var. germainii

Navicula salinarum

Navicula savannahiana

Navicula schoenfeldii

Navicula schroeteri var. escambia

Navicula scutiformis

Navicula secreta var. apiculata

Navicula secura

Navicula seminuloides

Navicula seminulum

Navicula seminulum var. intermedia

Navicula sp.

Navicula subarvensis

Navicula subfasciata
0.83

0.33

0.32

0.98

2.04

3.16

8.83

15.5

0.32

3.82

12.8

0.33

0.33

0.16

3.66

0.53

0.33

0.16

2.33

3.01

0.66

0.66

0.5

19.5

0.33

0.33

0.33

1.28

3.6

0.57

1.5

0.33

0.32

0.81

0.72

2.46

7.66

0.31

0.33

1.3

6.03

1.33

21.5

0.66

5.34

9.83

5.28

3.84

1.36

0.16 
Table 27. Algal taxa and their maximum relative abundance in quantitative samples collected in the Santee River Basin and Coastal Drainages study unit (Continued)

[Maximum relative abundance is the highest percentage of the total number of algal cells found in the sample set, and indicates the relative dominance of the taxon within the sample set. Collections of algal species include all quantitative and qualitative collections in the study unit. Blue-green algae, Cyanobacterium; ---, abundance too low to record]

\section{Algal group}

Algal taxon name

\section{Number of samples in which organism was identified}

\section{Maximum relative abundance (percent)}

Diatoms

Diatoms

Diatoms

Diatoms

Diatoms

Diatoms

Diatoms

Diatoms

Diatoms

Diatoms

Diatoms

Diatoms

Diatoms

Diatoms

Diatoms

Diatoms

Diatoms

Diatoms

Diatoms

Diatoms

Diatoms

Diatoms

Diatoms

Diatoms

Diatoms

Diatoms

Diatoms

Diatoms

Diatoms

Diatoms

Diatoms

Diatoms

Diatoms

Diatoms

Diatoms

Diatoms

Diatoms

Diatoms

Diatoms

Diatoms

Diatoms

Diatoms

Diatoms

Diatoms

Diatoms

Diatoms

Diatoms

Diatoms

Diatoms

Diatoms
Navicula subhamulata

Navicula subrhyncocephala

Navicula suhtilissima

Navicula symmetrica

Navicula tantula

Navicula tenelloides

Navicula tenera

Navicula thienemannii

Navicula tripunctata

Navicula tripunctata var. schizonemoides

Navicula utermoehlii

Navicula viridula

Navicula viridula var. linearis

Navicula viridula var. rostellata

Navicula vitabunda

Neidium affine

Neidium affine var. amphirhynchus

Neidium affine var. ceylonicum

Neidium affine var. longiceps

Neidium affine var. undulatum

Neidium alpinum

Neidium apiculatum

Neidium bisulcatum

Neidium cf. clementoides

Neidium cf. hankensis

Neidium cf. hermanii

Neidium dubium var.

Neidium hercynicum

Neidium hercynicum form. subrostratum

Neidium iridis

Neidium ladogense var. densestriatum

Neidium productum

Neidium $\mathrm{sp}$.

Nitzschia accommodata

Nitzschia acicularis

Nitzschia agnita

Nitzschia amphibia

Nitzschia bergii

Nitzschia brevissima

Nitzschia calida

Nitzschia capitellata

Nitzschia capitellata var. siberica

Nitzschia cf. aerophila

Nitzschia cf. capitellata

Nitzschia cf. frustulum var. perminuta

Nitzschia cf. obsidialis

Nitzschia cf. plana

Nitzschia cf. punctata var. coarctata

Nitzschia cf. sublineais

Nitzschia cf. sundaensis
1

3

1

14

2

7

1

1

3

5

I

6

3

1

2

8

1

3

1

9

1

2

1

3

1

1

8

4

1
0.32

1.33

0.34

12.4

0.92

5.66

0.16

0.16

1.83

1

0.66

0.31

0.5

1.31

0.34

0.65

0.63

0.3

0.33

0.5

6.83

0.66

0.71

0.32

0.98

2.43

0.33

0.66

0.66

0.16

0.5

0.34

0.32

2.04

3.31

0.33

11.2

1.33

0.5

0.66

1.66

0.31

1.43

0.49

0.57

1.38

0.16

0.34

0.65

0.64 
Table 27. Algal taxa and their maximum relative abundance in quantitative samples collected in the Santee River Basin and Coastal Drainages study unit (Continued)

[Maximum relative abundance is the highest percentage of the total number of algal cells found in the sample set, and indicates the relative dominance of the taxon within the sample set. Collections of algal species include all quantitative and qualitative collections in the study unit. Blue-green algae, Cyanobacterium; ---, abundance too low to record]

Algal group

Algal taxon name

\section{Number of samples in which organism was identified}

\section{Maximum relative abundance (percent)}

\begin{tabular}{|c|c|c|c|}
\hline Diatoms & Nitzschia cf. tarda & 1 & 1.95 \\
\hline Diatoms & Nitzschia clausii & 9 & 1.66 \\
\hline Diatoms & Nitzschia compressa & 1 & 0.16 \\
\hline Diatoms & Nitzschia constricta & 1 & 0.33 \\
\hline Diatoms & Nitzschia constricta var. subconstricta & 2 & 1.66 \\
\hline Diatoms & Nitzschia diserta & 1 & 0.32 \\
\hline Diatoms & Nitzschia dissipata & 15 & 4.08 \\
\hline Diatoms & Nitzschia dissipata var. media & 3 & 0.33 \\
\hline Diatoms & Nitzschia filiformis & 7 & 3.36 \\
\hline Diatoms & Nitzschia flexa & 1 & 1.66 \\
\hline Diatoms & Nitzschia fonticola & 8 & 1.98 \\
\hline Diatoms & Nitzschia frustulum & 12 & 3.8 \\
\hline Diatoms & Nitzschia frustulum var. perminuta & 15 & 1.5 \\
\hline Diatoms & Nitzschia gracilis & 11 & 10 \\
\hline Diatoms & Nitzschia hantzschiana & 2 & 0.33 \\
\hline Diatoms & Nitzschia inconspicua & 1 & 0.33 \\
\hline Diatoms & Nitzschia intermedia & 5 & 0.33 \\
\hline Diatoms & Nitzschia linearis & 16 & 6 \\
\hline Diatoms & Nitzschia microcephala & 1 & 0.33 \\
\hline Diatoms & Nitzschia nana & 2 & 0.32 \\
\hline Diatoms & Nitzschia obtusa var. scalpelliformis & 2 & 0.33 \\
\hline Diatoms & Nitzschia obtusa & 2 & 0.65 \\
\hline Diatoms & Nitzschia palea & 37 & 18 \\
\hline Diatoms & Nitzschia paleacea & 2 & 0.64 \\
\hline Diatoms & Nitzschia pusilla & 3 & 1.66 \\
\hline Diatoms & Nitzschia radicula var. rostrata & 2 & 1.63 \\
\hline Diatoms & Nitzschia recta & 11 & 2.16 \\
\hline Diatoms & Nitzschia rostellata & 1 & 0.86 \\
\hline Diatoms & Nitzschia scalaris & 1 & 0.33 \\
\hline Diatoms & Nitzschia sigma & 1 & 0.33 \\
\hline Diatoms & Nitzschia sigmoidea & 1 & 0.66 \\
\hline Diatoms & Nitzschia silicula & 1 & 0.33 \\
\hline Diatoms & Nitzschia similis & 1 & 0.65 \\
\hline Diatoms & Nitzschia sp. & 6 & 0.68 \\
\hline Diatoms & Nitzschia stagnorum & 1 & 0.32 \\
\hline Diatoms & Nitzschia subacicularis & 1 & 0.16 \\
\hline Diatoms & Nitzschia subcommunis & 1 & 0.8 \\
\hline Diatoms & Nitzschia tarda & 4 & 0.97 \\
\hline Diatoms & Nitzschia terrestris (Peterson) & 1 & 0.5 \\
\hline Diatoms & Nitzschia thermalis var. minor & 1 & 0.28 \\
\hline Diatoms & Nitzschia tryblionella var. debilis & 7 & 2.16 \\
\hline Diatoms & Nitzschia tryblionella var. levidensis & 2 & 0.83 \\
\hline Diatoms & Nitzschia tryblionella var. victoriae & 3 & 1.63 \\
\hline Diatoms & Nitzschia tubicola & 1 & 0.16 \\
\hline Diatoms & Opephora martyi & 5 & 1.27 \\
\hline Diatoms & Opephora olsenii & 1 & 0.16 \\
\hline Diatoms & Peronia fibula & 1 & 0.33 \\
\hline Diatoms & Pinnularia abaujensis & 2 & 0.32 \\
\hline Diatoms & Pinnularia abaujensis var. cf. subundulata & 1 & 2.55 \\
\hline Diatoms & Pinnularia acrosphaeria. & 7 & 1.66 \\
\hline
\end{tabular}


Table 27. Algal taxa and their maximum relative abundance in quantitative samples collected in the Santee River Basin and Coastal Drainages study unit (Continued)

[Maximum relative abundance is the highest percentage of the total number of algal cells found in the sample set, and indicates the relative dominance of the taxon within the sample set. Collections of algal species include all quantitative and qualitative collections in the study unit. Blue-green algae, Cyanobacterium; ---, abundance too low to record]

Algal group
Algal taxon name

\section{Number of samples in which organism was identified}

\section{Maximum relative abundance (percent)}

Diatoms

Diatoms

Diatoms

Diatoms

Diatoms

Diatoms

Diatoms

Diatoms

Diatoms

Diatoms

Diatoms

Diatoms

Diatoms

Diatoms

Diatoms

Diatoms

Diatoms

Diatoms

Diatoms

Diatoms

Diatoms

Diatoms

Diatoms

Diatoms

Diatoms

Diatoms

Diatoms

Diatoms

Diatoms

Diatoms

Diatoms

Diatoms

Diatoms

Diatoms

Diatoms

Diatoms

Diatoms

Diatoms

Diatoms

Diatoms

Diatoms

Diatoms

Diatoms

Diatoms

Diatoms

Diatoms

Diatoms

Diatoms

Diatoms

Diatoms
Pinnularia acrosphaeria var.

Pinnularia appendiculata

Pinnularia biceps

Pinnularia borealis

Pinnularia braunii

Pinnularia braunii var. amphicephala

Pinnularia brebissonii.

Pinnularia cf. rivularis

Pinnularia ef. subcapitata

Pinnularia divergens

Pinnularia latevittata

Pinnularia mesogongyla

Pinnularia mesolepta

Pinnularia microstauron

Pinnularia obscura

Pinnularia ruttneri

Pinnularia sp.

Pinnularia sp.?

Pinnularia subcapitata

Pinnularia subcapitata var. paucistriata

Pinnularia substomatophora

Pinnularia termitina

Pinnularia viridis.

Reimeria sinuata

Rhoicosphenia curvata

Rhopalodia gibba

Rhopalodia gibberula

Rhopalodia gibberula var. vanheurckii

Stauroneis anceps

Stauroneis anceps form. americana

Stauroneis anceps form. gracilis

Stauroneis borrichii

Stauroneis cf. prominula

Stauroneis cf. thermicola

Stauroneis ignorata

Stauroneis ignorata var. rupestris

Stauroneis kriegeri

Stauroneis livingstonii

Stauroneis phoenicenteron

Stauroneis smithii

Stauroneis smithii var. incisa

Stauroneis sp.

Stauroneis staurolineata

Stauroneis thermicola

Stenopterobia densestriata

Stephanodiscus agassizensis

Surirella amphioxys

Surirella angusta

Surirella brebissonii

Surirella delicatissima
1

2

6

1

9

1

6

11

8

1

13

3

2

3

2

2

5

1

1

1

5

3

5

2

1

2

1

3

3

1

5

9

3

3

1

6

8

2
0.16

0.63

0.65

0.16

0.5

1.83

0.86

0.32

0.32

0.5

2.83

22.2

0.16

0.5

0.83

1.31

1.27

0.65

2.07

1.16

0.57

4.29

0.16

0.16

6.83

0.16

0.33

0.66

0.5

1.64

0.66

0.57

0.32

0.31

0.64

0.33

0.96

0.32

0.66

1.6

0.33

0.32

0.16

0.66

3

0.16

0.16

4.43

0.31

2.16 
Table 27. Algal taxa and their maximum relative abundance in quantitative samples collected in the Santee River Basin and Coastal Drainages study unit (Continued)

[Maximum relative abundance is the highest percentage of the total number of algal cells found in the sample set, and indicates the relative dominance of the taxon within the sample set. Collections of algal species include all quantitative and qualitative collections in the study unit. Blue-green algae, Cyanobacterium; ---, abundance too low to record]

\section{Algal group}

Algal taxon name

\section{Number of samples in which organism was identified}

\section{Maximum relative abundance (percent)}

Diatoms

Diatoms

Diatoms

Diatoms

Diatoms

Diatoms

Diatoms

Diatoms

Diatoms

Diatoms

Diatoms

Diatoms

Diatoms

Diatoms

Diatoms

Diatoms

Diatoms

Diatoms

Diatoms

Diatoms

Diatoms

Diatoms

Diatoms

Diatoms

Diatoms

Diatoms

Diatoms

Diatoms

Diatoms

Diatoms

Diatoms

Dinoflagellates

Euglenoids

Euglenoids

Euglenoids

Euglenoids

Euglenoids

Euglenoids

Euglenoids

Green algae

Green algae

Green algae

Green algae

Green algae

Green algae

Green algae

Green algae

Green algae

Green algae

Green algae
Surirella elegans

Surirella guatimalensis

Surirella linearis var. constricta

Surirella minuta

Surirella ovata var. africana

Surirella ovata var. pinnata

Surirella robusta var. splendida form. constricta

Surirella sp.

Surirella stalagma

Surirella suecica

Surirella tenera

Surirella tenera var. nervosa

Synedra acus

Synedra amphicephala var. austriaca

Synedra cf. amphicephala

Synedra cf. famelica

Synedra fasciculata

Synedra filiformis var, exilis

Synedra goulardi

Synedra minuscula

Synedra parasitica

Synedra pulchella var. lacerata

Synedra rumpens

Synedra rumpens var. familiaris

Synedra rumpens var. meneghiniana

Synedra sp.

Synedra ulna

Synedra ulna var. danica

Tabellaria fenestrata

Tabellaria flocculosa

Thalassiosira weissflogii

Peridinium sp.

Euglena sp.

Phacus sp.

Trachelomonas cylindrica

Trachelomonas girardiana

Trachelomonas hispida

Trachelomonas sp.

Trachelomonas volvocina

Actinastrum hantzschii

Ankistrodesmus falcatus

Chlamydomonas sp.

Cladophora glomerata

Closterium abruptum form angustissima

Closterium baillyanum

Closterium dianae

Closterium kutzingii

Closterium libellula

Closterium moniliferum

Closterium venus
0.66

0.16

0.15

0.16

0.3

1.2

0.34

0.32

2.61

0.65

1.63

1.36

0.95

0.32

0.43

0.31

0.16

0.16

0.49

1.83

0.33

0.66

2.46

1.8

1

15.3

9.66

0.5

3.16

3

0.83

2.3

1.31

0.55

0.32

0.32

0.33

0.49

1.46

---

3.35
1.89

0.65

-.

$-$

$--$

0.59 
Table 27. Algal taxa and their maximum relative abundance in quantitative samples collected in the Santee River Basin and Coastal Drainages study unit (Continued)

[Maximum relative abundance is the highest percentage of the total number of algal cells found in the sample set, and indicates the relative dominance of the taxon within the sample set. Collections of algal species include all quantitative and qualitative collections in the study unit. Blue-green algae, Cyanobacterium; ---, abundance too low to record]

\section{Algal group}

Algal taxon name
Green algae

Green algae

Green algae

Green algae

Green algae

Green algae

Green algae

Green algae

Green algae

Green algae

Green algae

Green algae

Green algae

Green algae

Green algae

Green algae

Green algae

Green algae

Green algae

Green algae

Green algae

Green algae

Green algae

Green algae

Green algae

Green algae

Green algae

Green algae

Green algae

Red algae

Red algae

Red algae

Unknown phylum

Unknown phylum

Unknown phylum

Unknown phylum

Yellow-green algae

Yellow-green algae

Yellow-green algae
Cosmarium botrytes

Cosmarium punctulatum

Cosmarium pyramidatum

Cosmarium reinschii

Euastrum binale var. gutwinskii

Euastrum inerme

Eudorina elegans

Gloeocystis sp.

Kirchneriella lunaris

Micrasterias crux-melitensis

Mougeotia sp.

Oedogonium sp.

Pediastrum biradiatum

Pediastrum boryanum

Pleurotaenium trabecula var. trabecula

Scenedesmus acuminatus

Scenedesmus acutus

Scenedesmus armatus

Scenedesmus ecornis

Scenedesmus quadricauda

Scenedesmus sp.

Spirogyra sp.

Staurastrum alternans

Staurastrum gladiosum

Staurastrum sp.

Tetraedron minimum

Tetrastrum staurogeniaeforme

Treubaria crassipina

Undetermined green coccoid (5-10 micron)

Audouinella sp.

Audouinella violacea

Batrachospermum sp.

Undetermined algal coccoid (3-5 micron)

Number of samples in which organism was identified

\section{Maximum relative abundance \\ (percent)}

Undetermined algal flagellate ( $<10$ micron)

Undetermined algal flagellate (>10 micron)

Undetermined algal sp.

Dinobryon sp.

Undetermined chrysophyte sp.

Vaucheria sp.
$--$

$\cdots$

0.32

$--$

0.32

0.65

0.32

0.32

$--$

0.98

0.56

0.32

0.97

$---$

0.64

0.65

0.97

1.93

5.16

0.32

$--$

0.33

$--$

1.59

0.64

0.59

0.61

3.03

1.85

$--$

3.03

33.2

1.99

5.17

0.65 
ํ.广ิ廿0

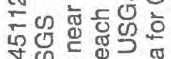

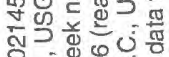

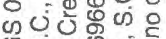

क ज

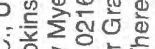

Z우응욜

बi

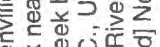

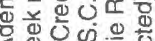

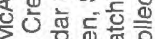

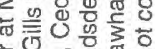

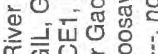

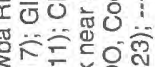

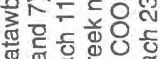

o 0 \%

눈

돈 कि

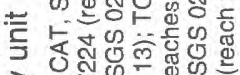

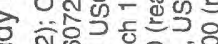

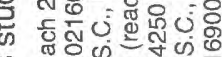

\& 0

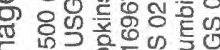

뜬

牙的政元

ब

ज़

Oํ.

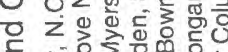

元

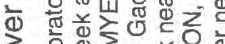

议

ఖ

何 \%

(1) 造之

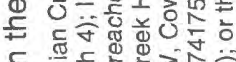

든

叫

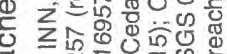

๘ $=$ 청 त

E

1.

部

o ju心

No

क क Eु O

क

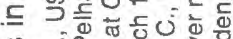

\&

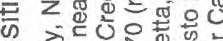

ब.

O

过

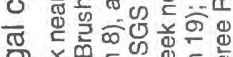

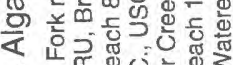

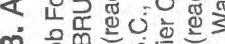

施

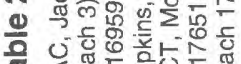

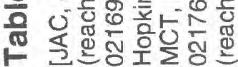

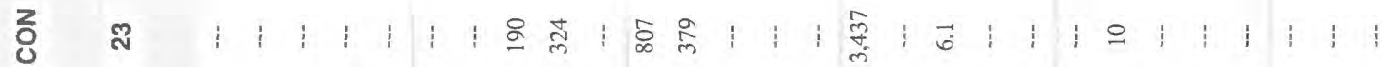

㩊

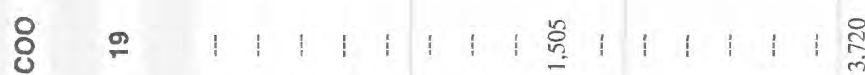

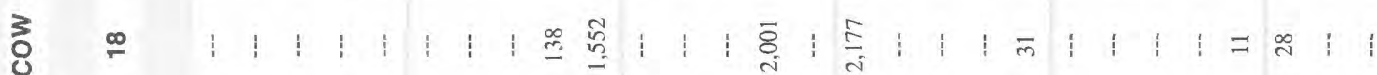

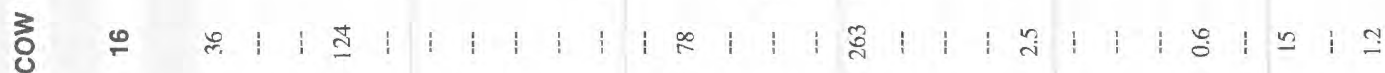

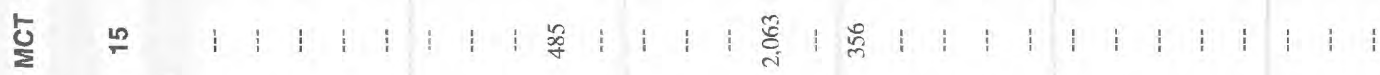

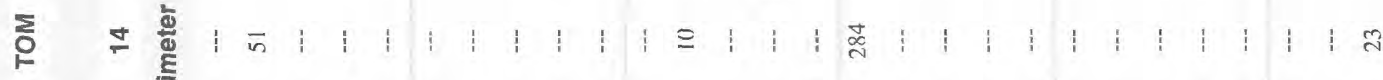

芋 응

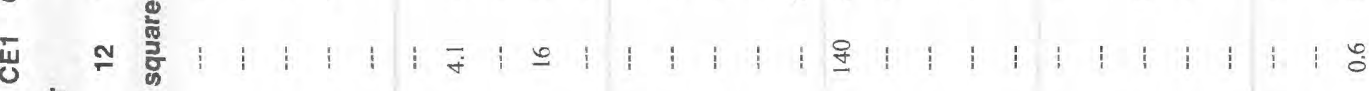

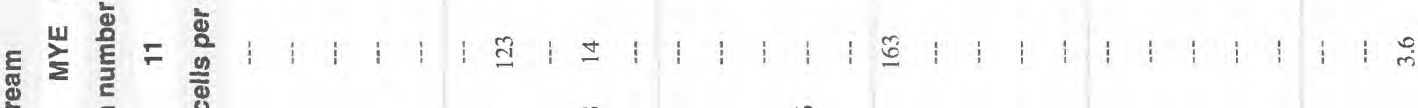

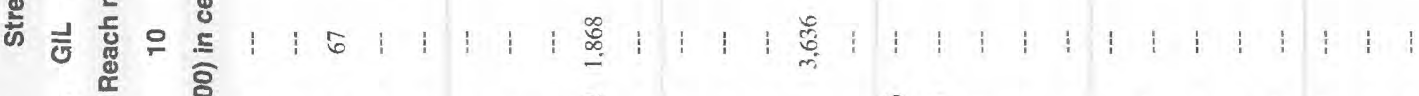

吉

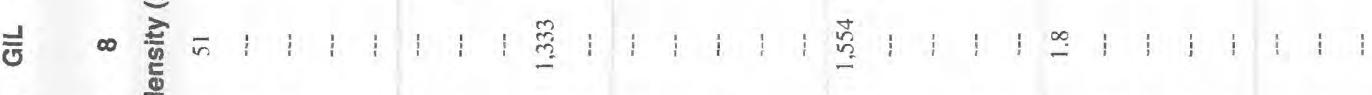

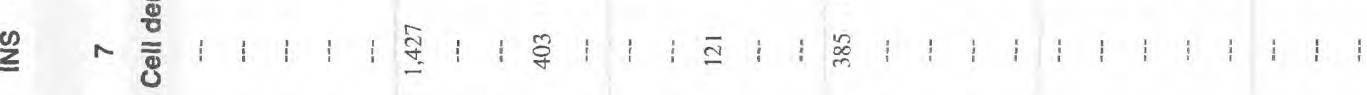

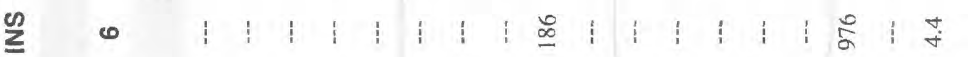

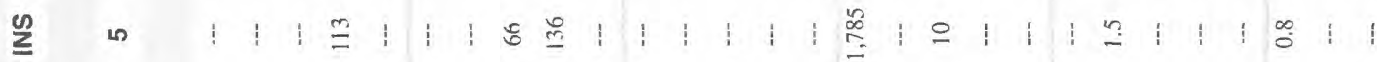

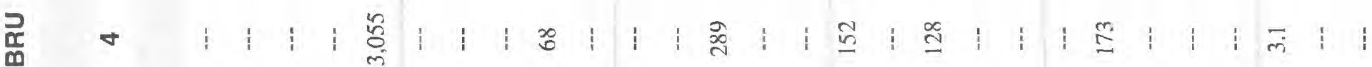

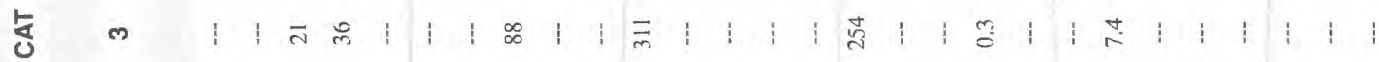

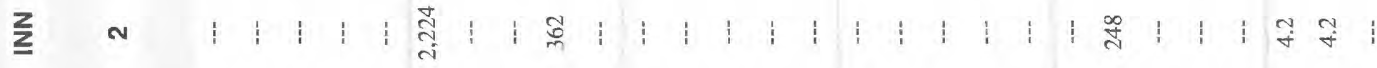

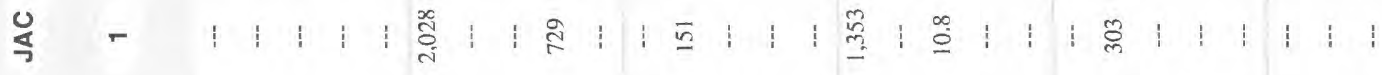

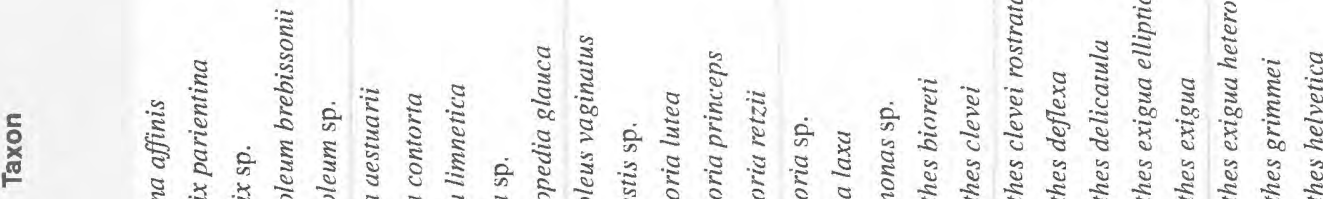

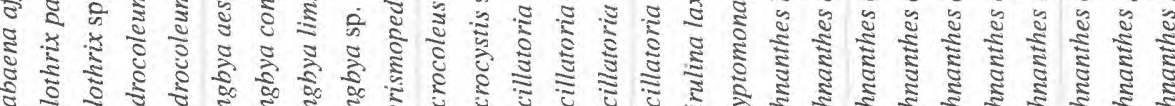

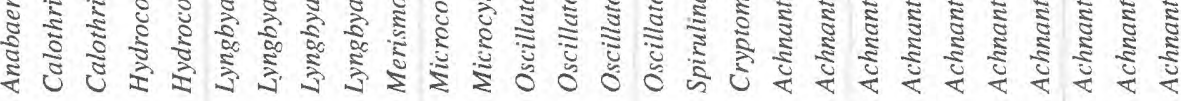

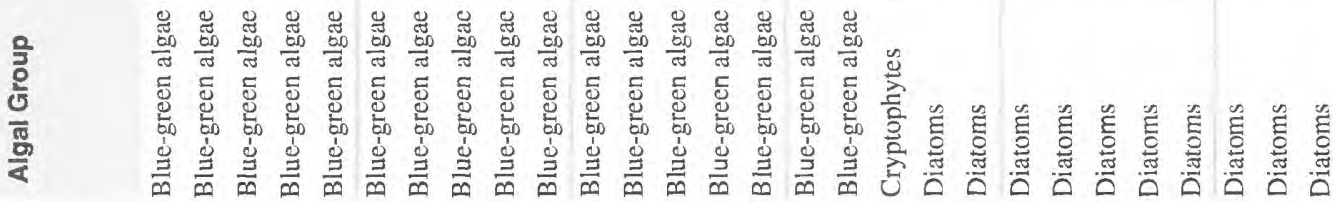




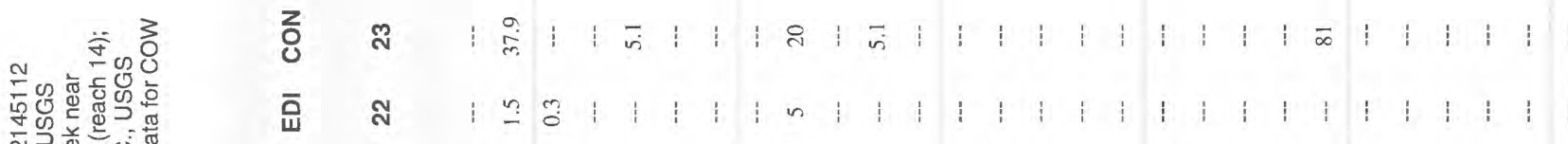

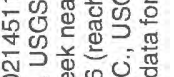
किण छ Uु. 3 舟 0.

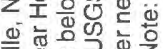

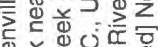

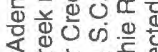

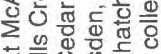

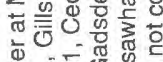

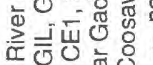

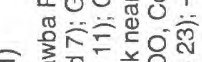

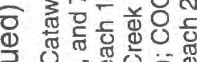

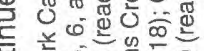

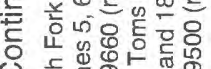

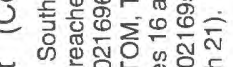

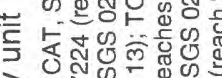

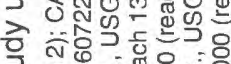

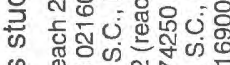
\% 는

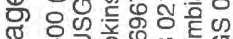

는 匹 O क्ष 范 U Uz心

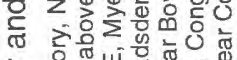

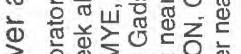
近

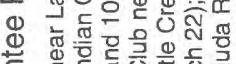

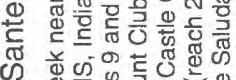
心 凹

๘

¿ ¿ 宊 E

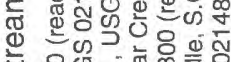
ठ유ำ

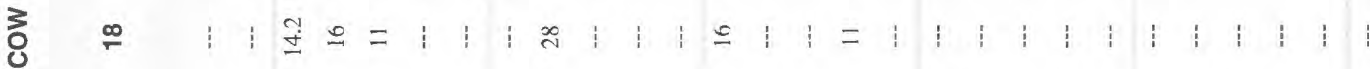
产 包 ò

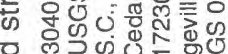

要

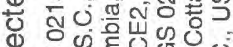

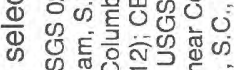

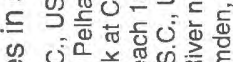

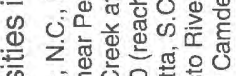
के

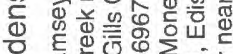
要 ब

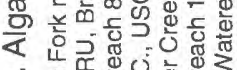
究 00000

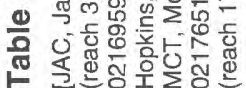

I)

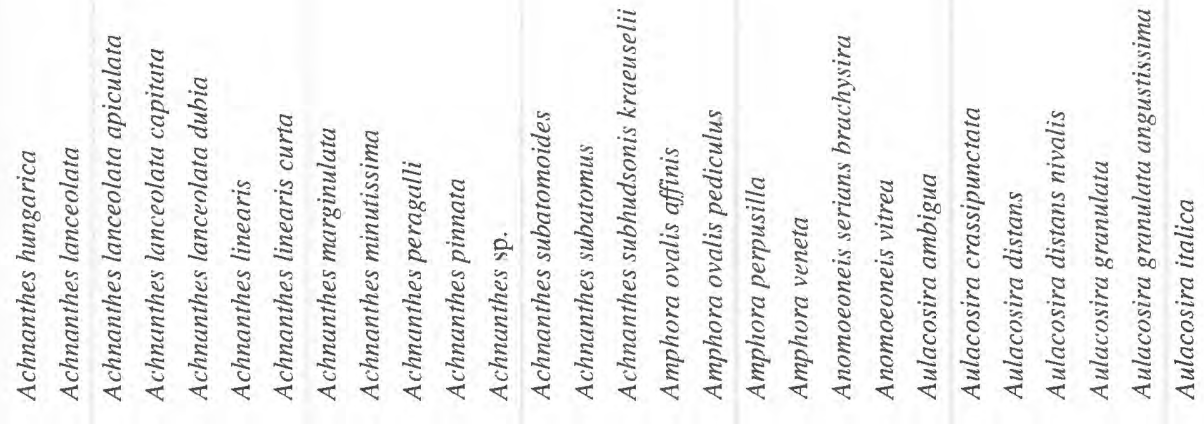




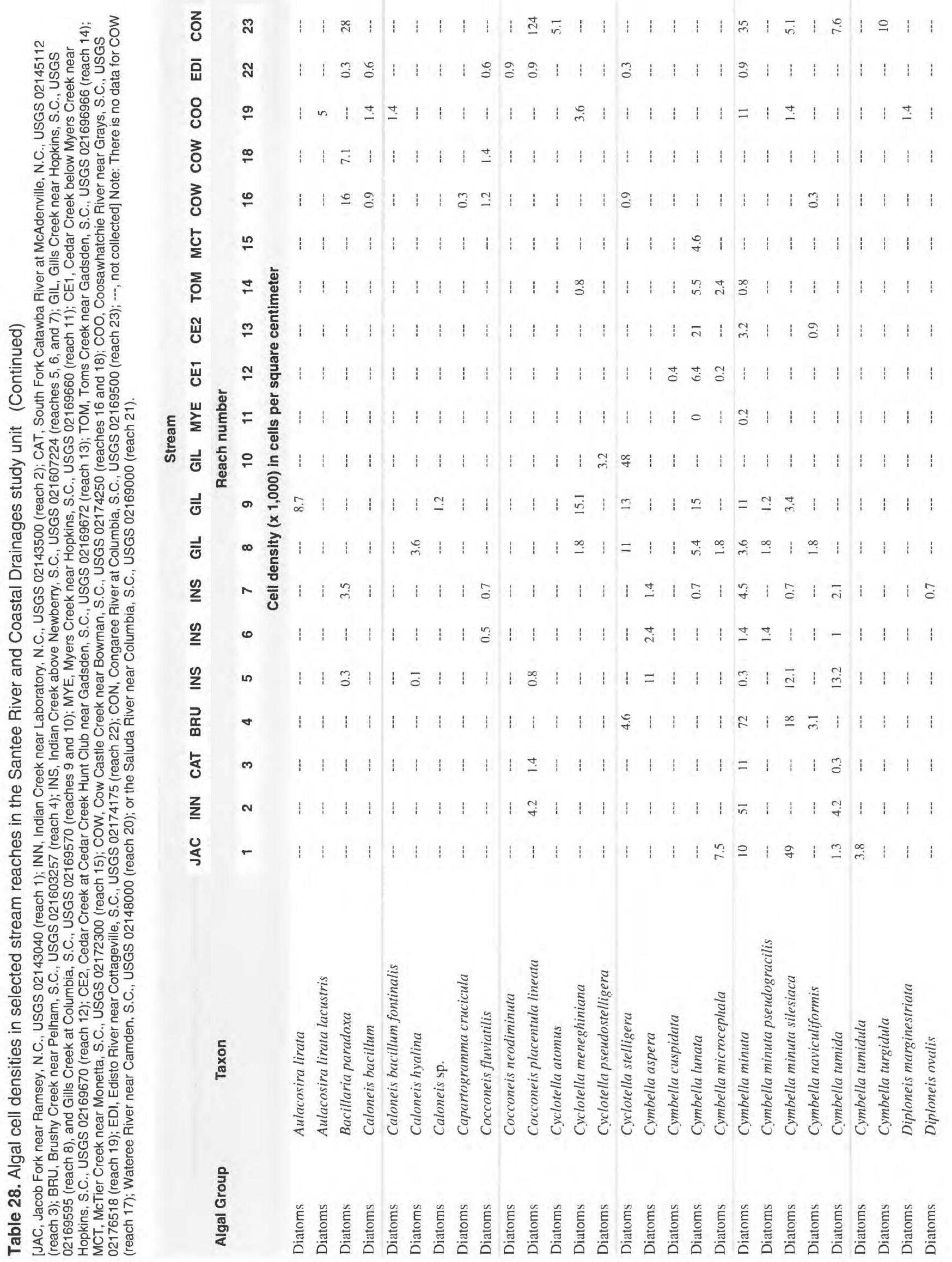




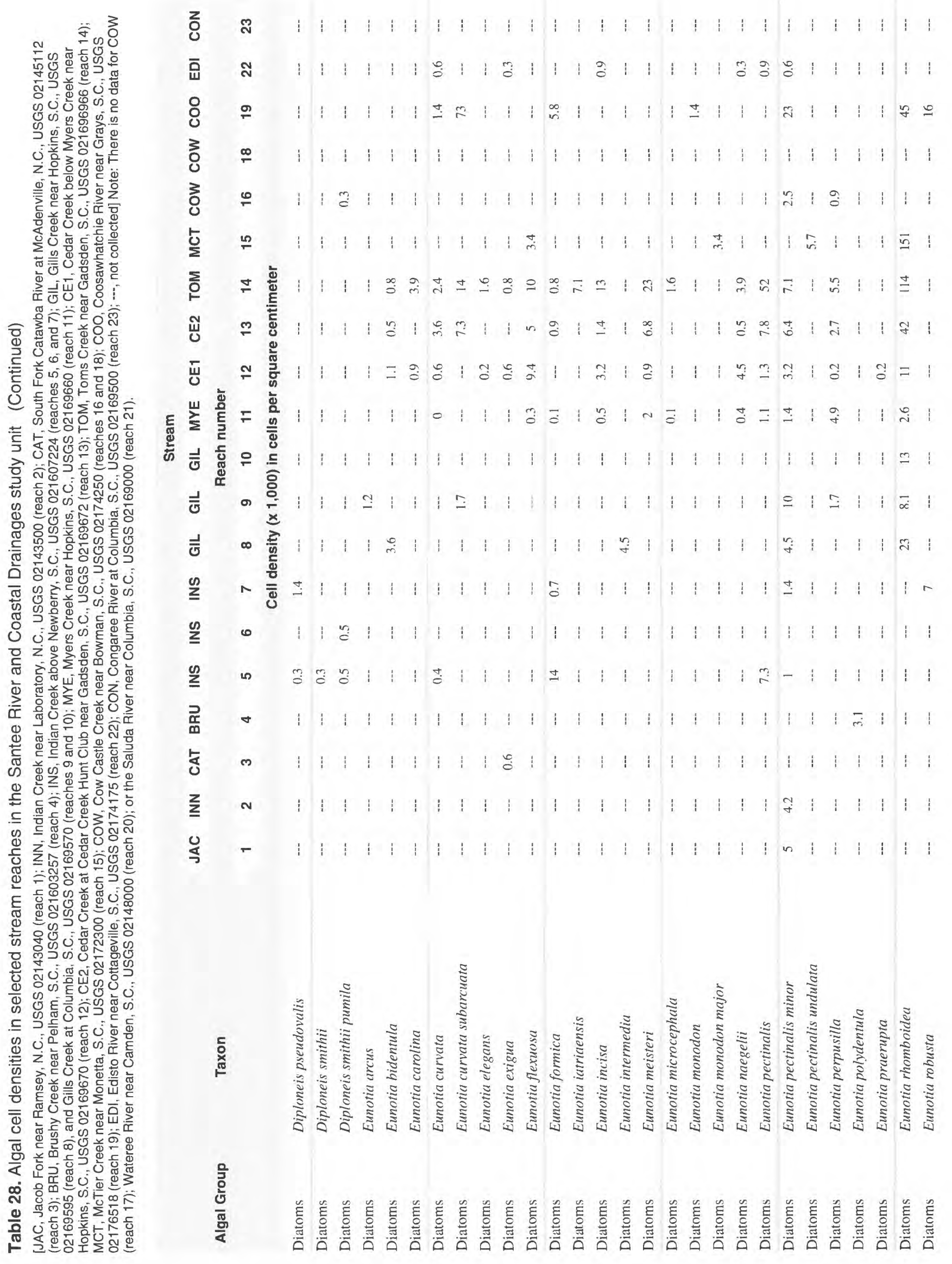




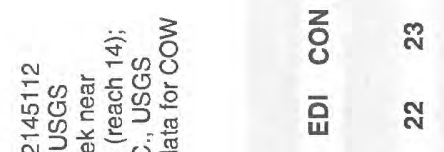

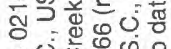

Oo $00 \%$

雪

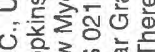

之오흥에 융.

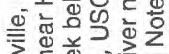

递记辛

웅은

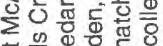

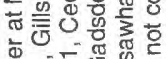

牙议

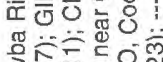

Oิ

品

당

U

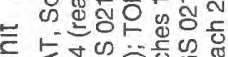

与ิ

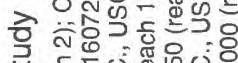

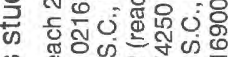

0

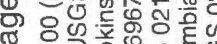

단

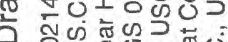

匹

की

סु

O

它

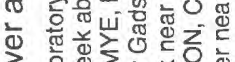

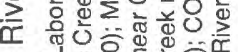

\&

究

की

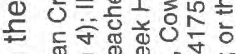

.

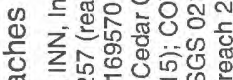

d

บิ ส

용

요

ठิ

เ

言

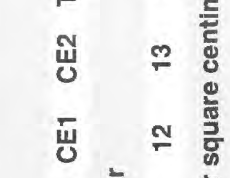

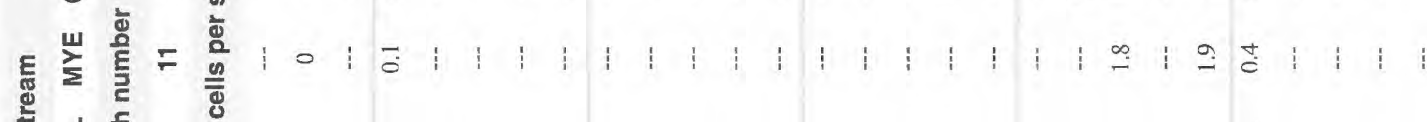

के

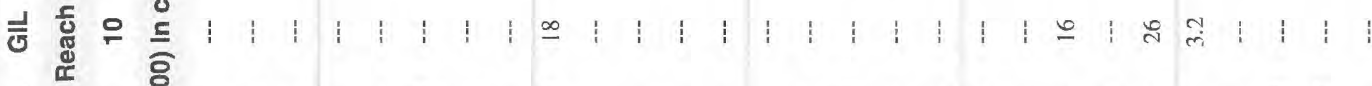
न

志 $\infty$

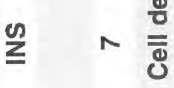

$\underline{n}$

$\underline{m}$

$\underline{n}$

ᄀ

萿

过

z N

造

E

d

क

要

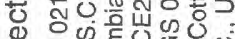

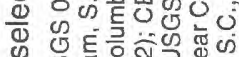

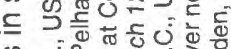

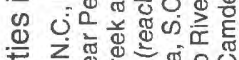

둔

议

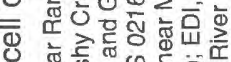

ब

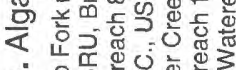

ง

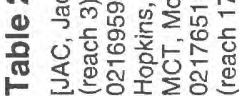

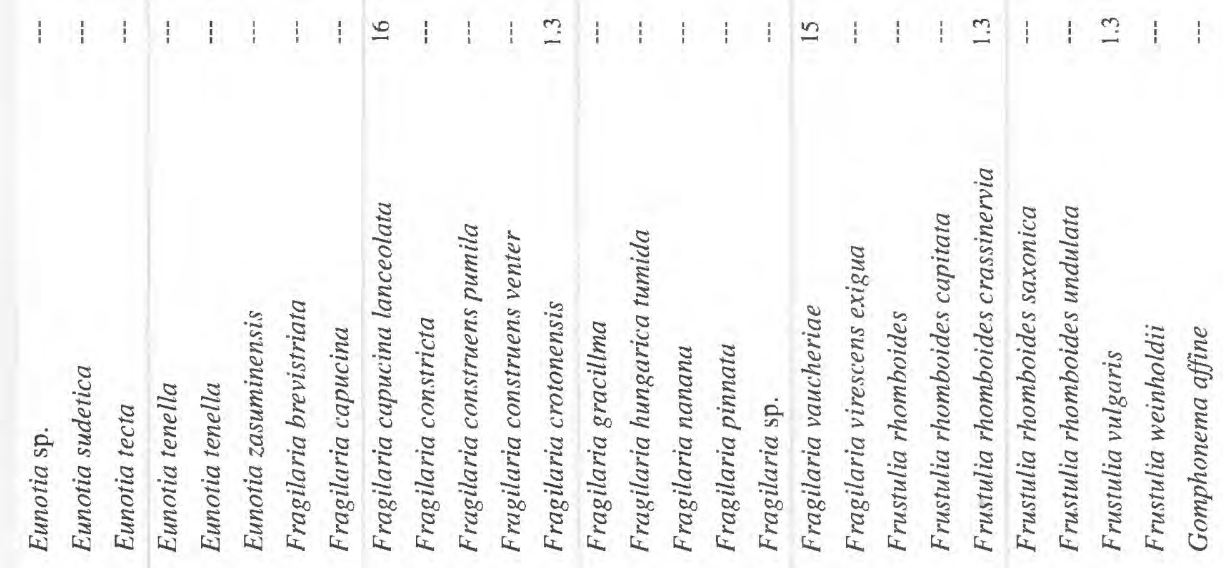




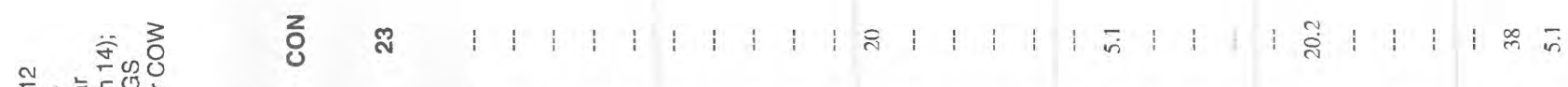

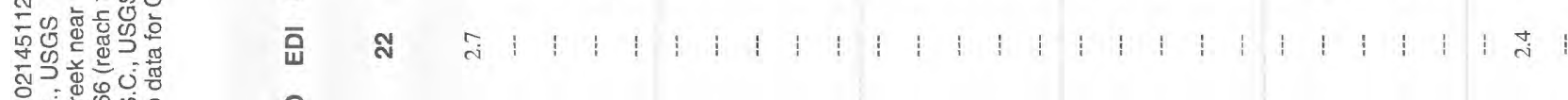

o $0 \%$

Do w o w w

Sis

之오응엉

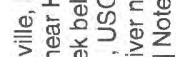

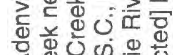

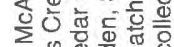

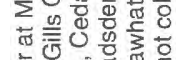

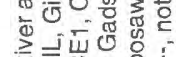

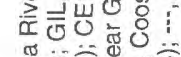

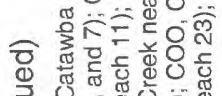

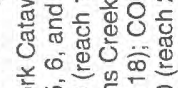

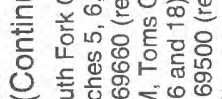

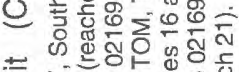

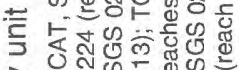

交

क

o)

\%

空

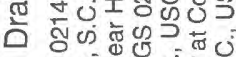

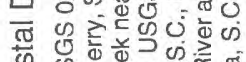

कू

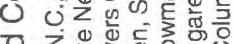

品

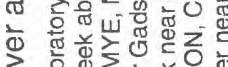

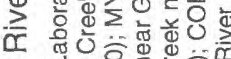

迹

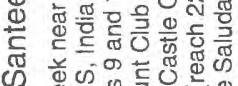

跑

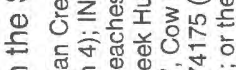

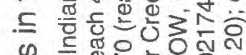

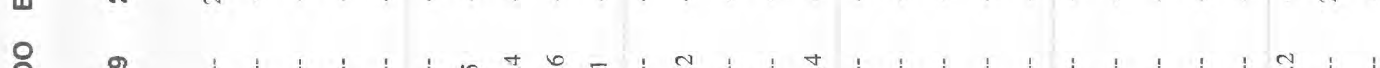

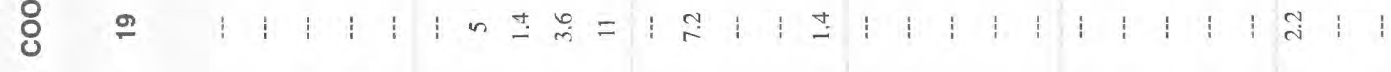

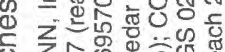

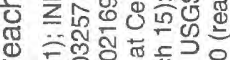

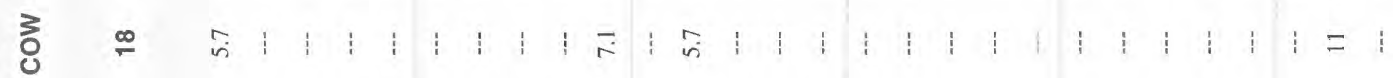

言

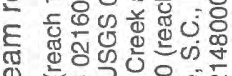

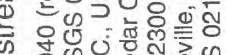

- ỡ

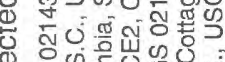

o

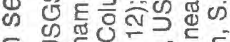

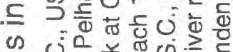

\&

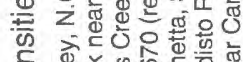

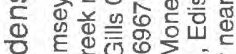

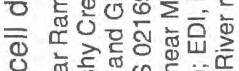

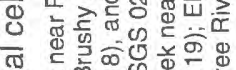

ত্

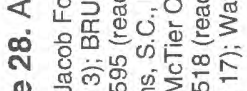

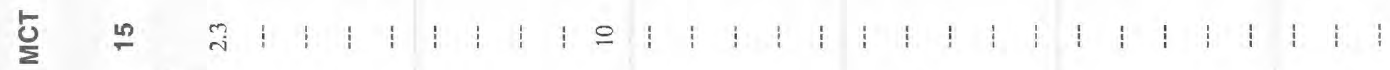

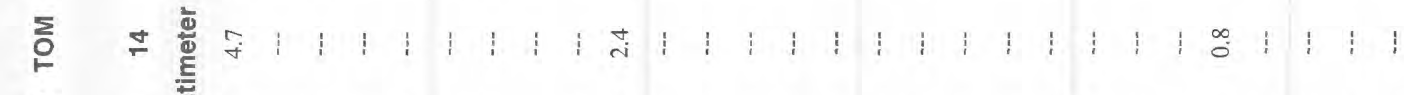

芩

ธ్ ㄷํำ

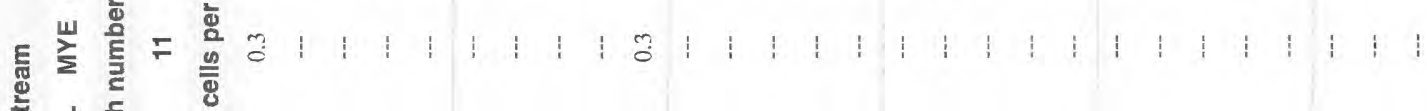

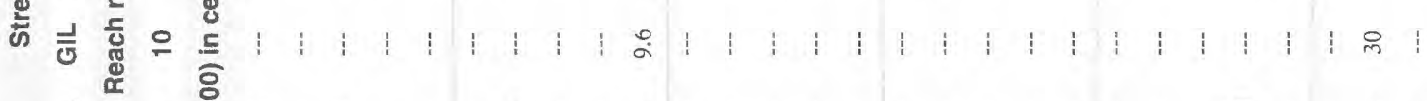

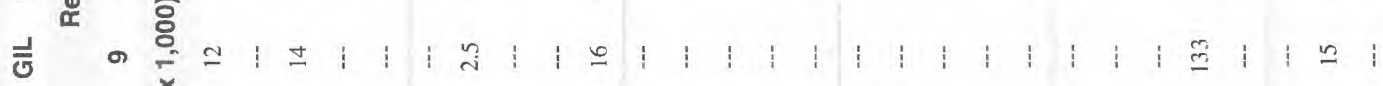

ప $\infty$ 蛋

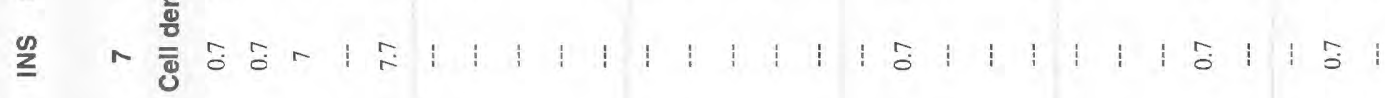

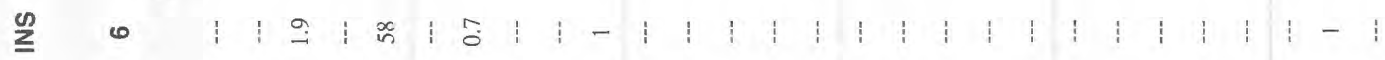

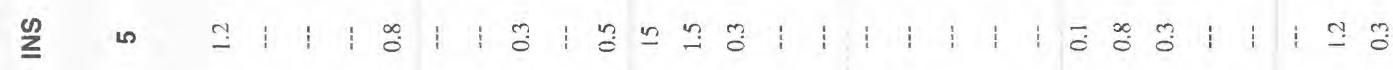

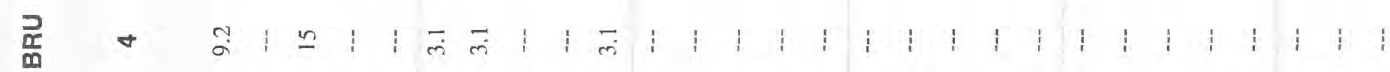

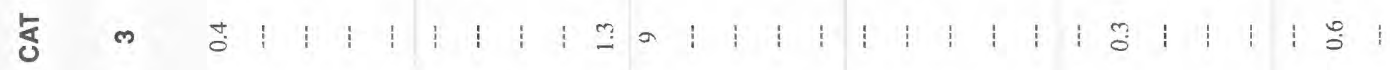

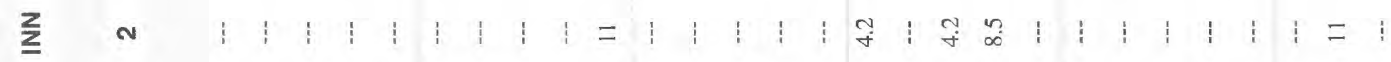

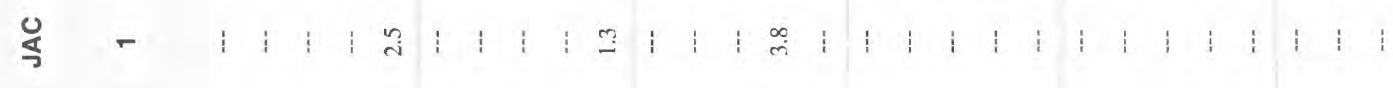

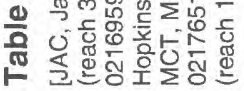

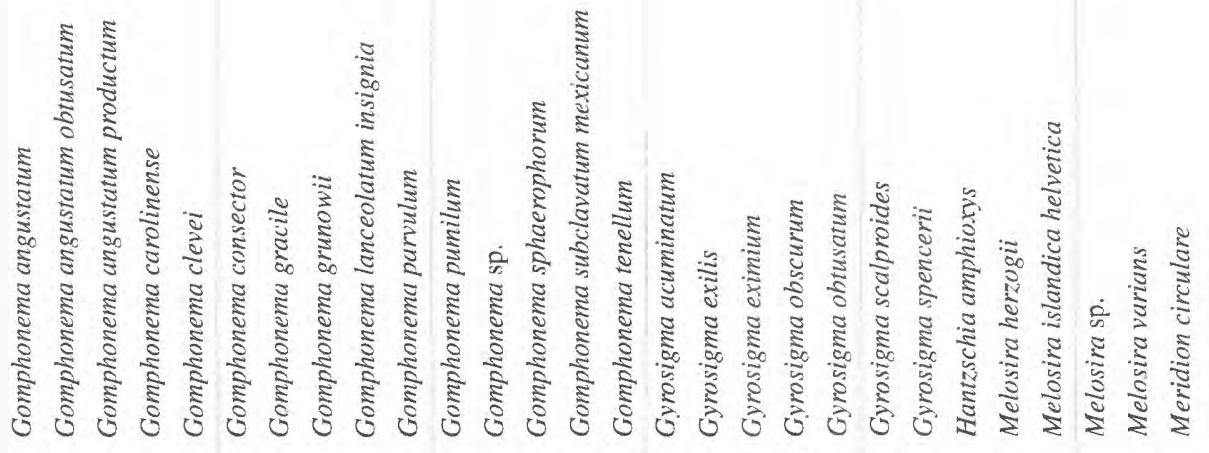

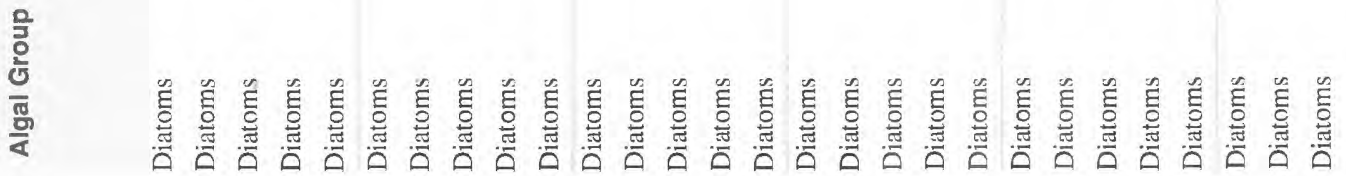


थ⿻上丨

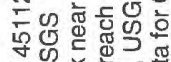

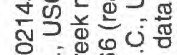

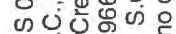

कु

$\supset$ v

i 홍

之오응웜

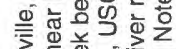

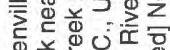
。

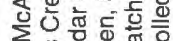

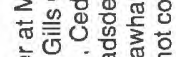

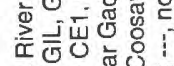

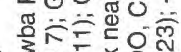

वิ

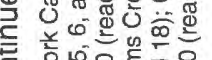
동 吠

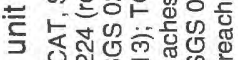

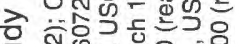

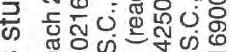

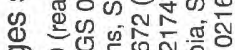

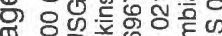

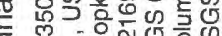

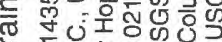
万人

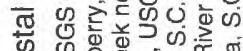
密 ठํ.

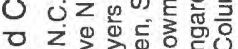
率 就

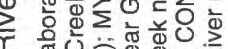
ง 

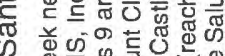

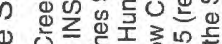

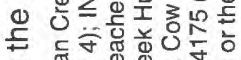

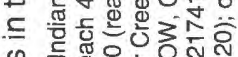
\&

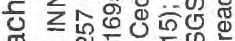
\& E ه

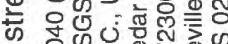
D 政以

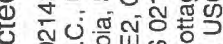
क ज हु잉 め

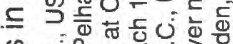
\& 0 员羊的文

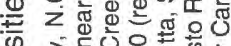

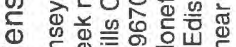
응 E

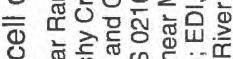
匹

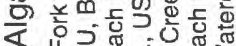
穴 N

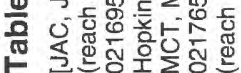

zo

ธ 8 웅

응

ㅇํㅇ

!

인 $\div$

要

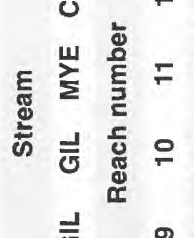

ज

光 $\infty$

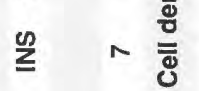

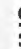

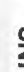

产

5

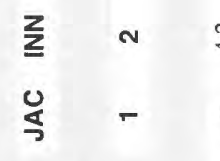
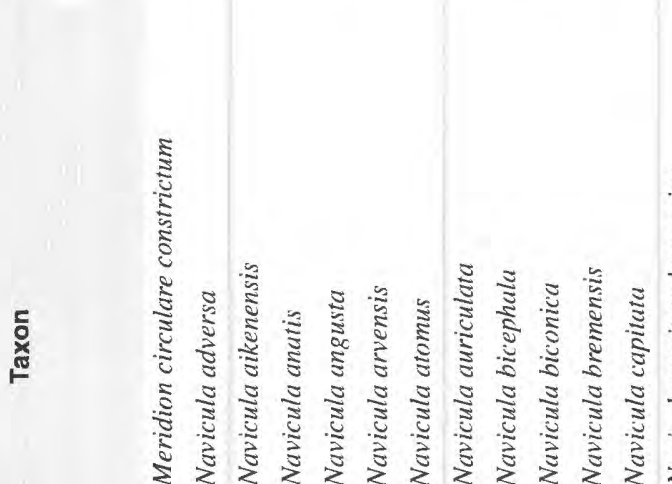

ฯ

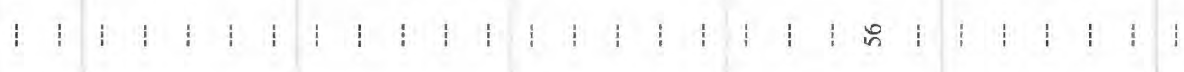

$\frac{\text { 웅 }}{\frac{2}{0}}$

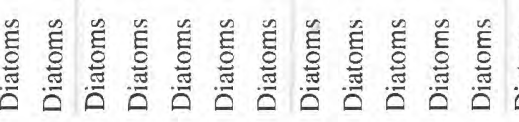

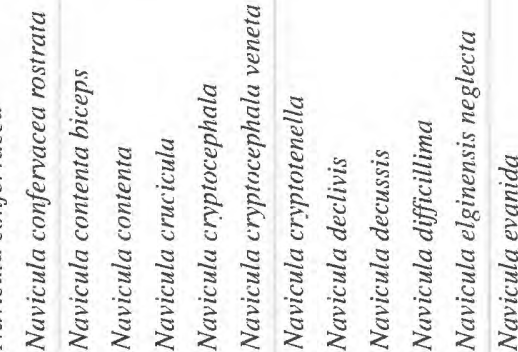




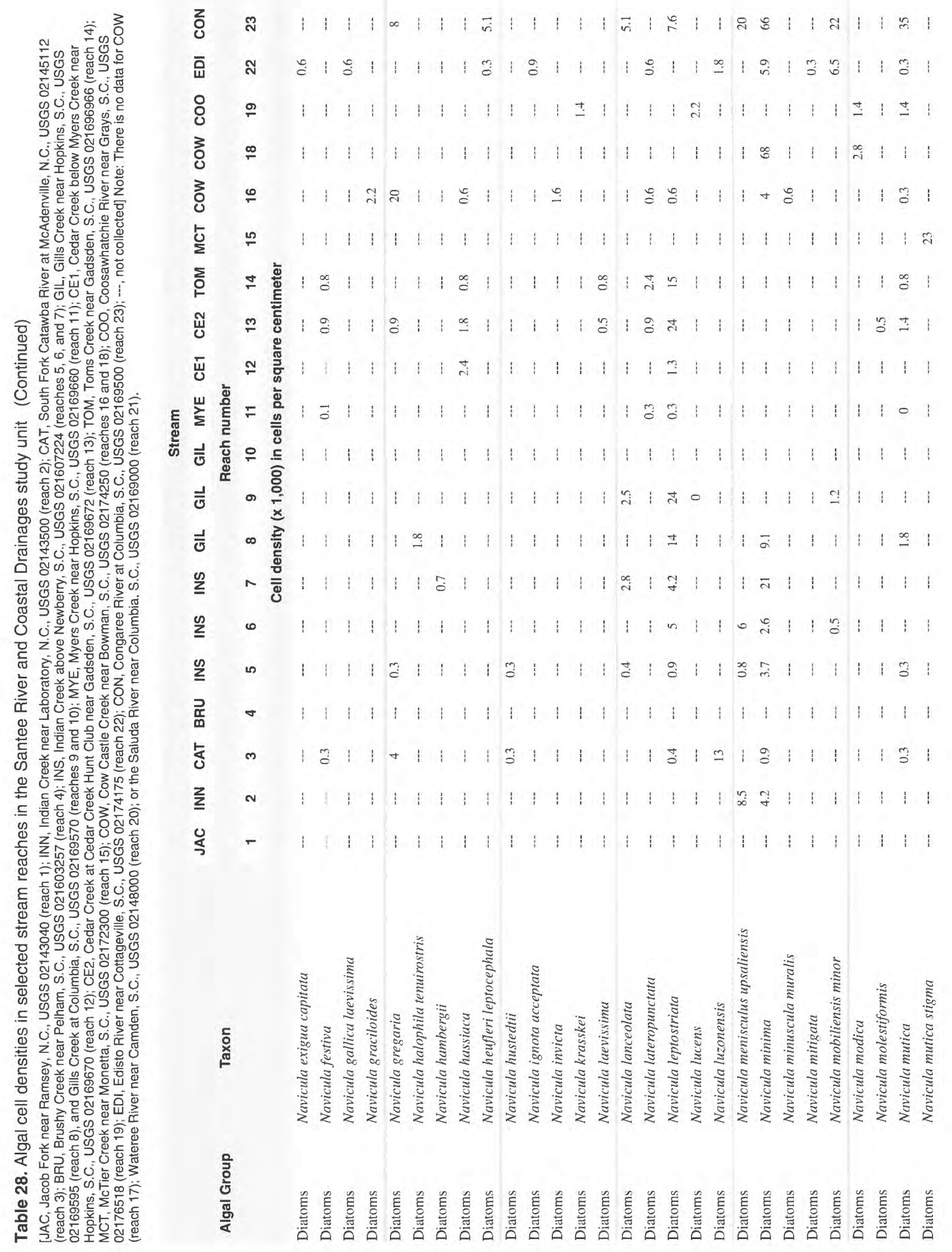




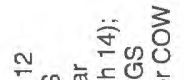

당워

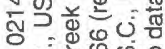

oํㅇ⿺ㅇ

们

$\supset$ 它

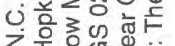

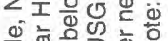

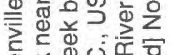

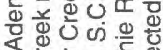

舟语

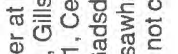

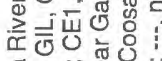

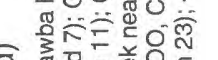

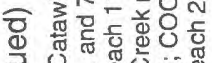

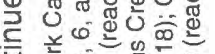

ㄷㅎㄴ

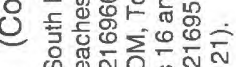

竞

흔

फ

\& 8000

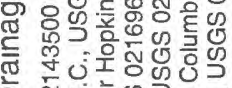

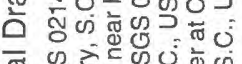

区

范

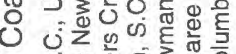

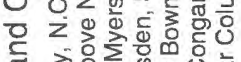

त र्ते

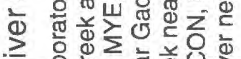

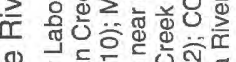

离

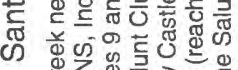

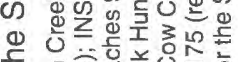

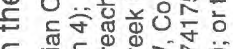

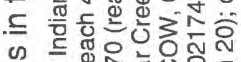

\&

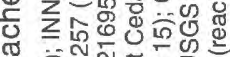

屯 $=0$

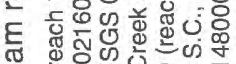

¿

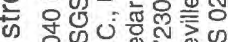

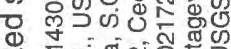

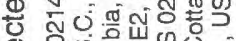

\%

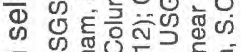

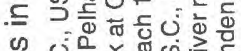

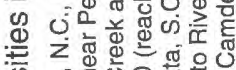

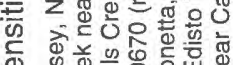

임

离

ब

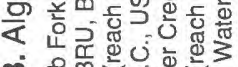

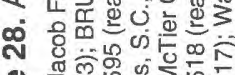

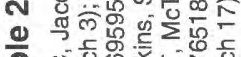

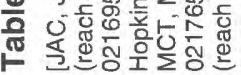

ठำ

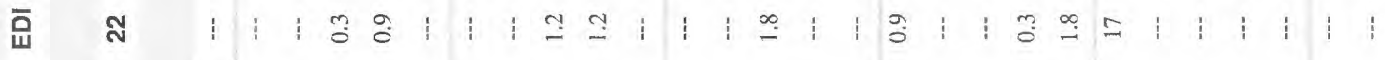

ठำ

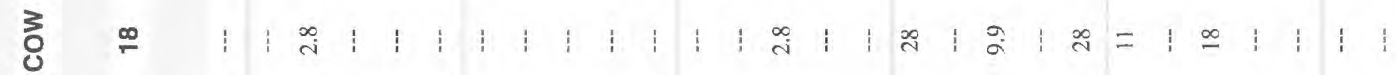

0
0

$\stackrel{5}{\vdots}$

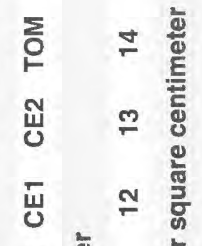

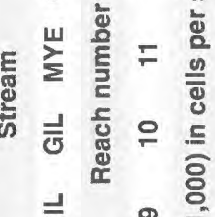

है

$\stackrel{\infty}{=} \infty$

वृ

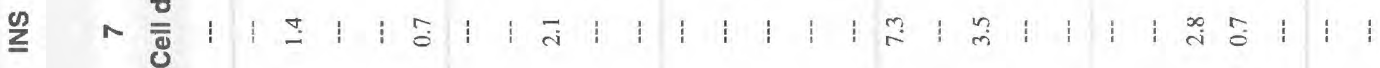

Љ

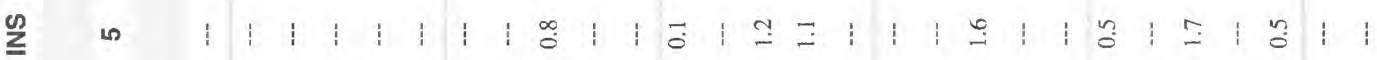

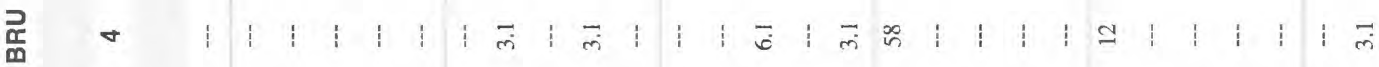

志 m

z

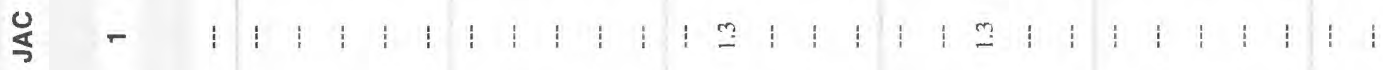

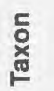

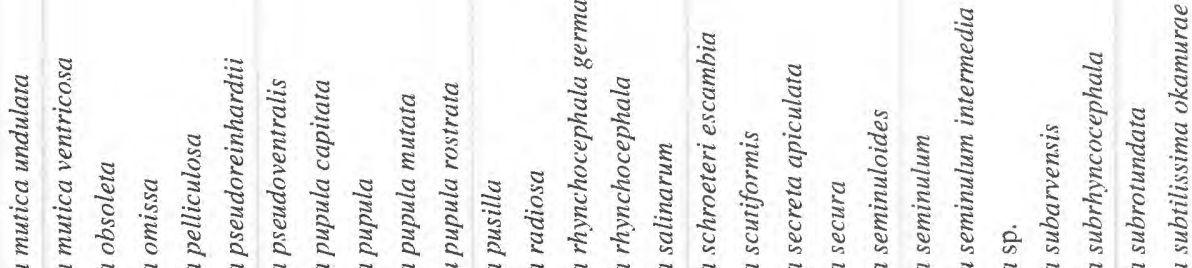

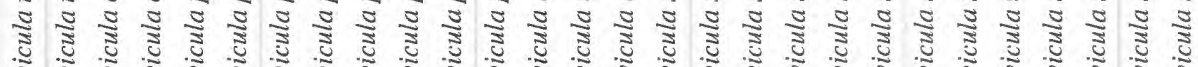

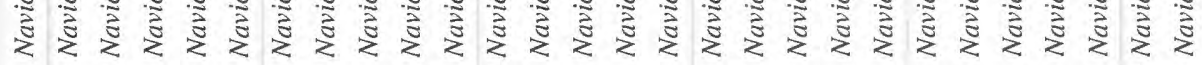




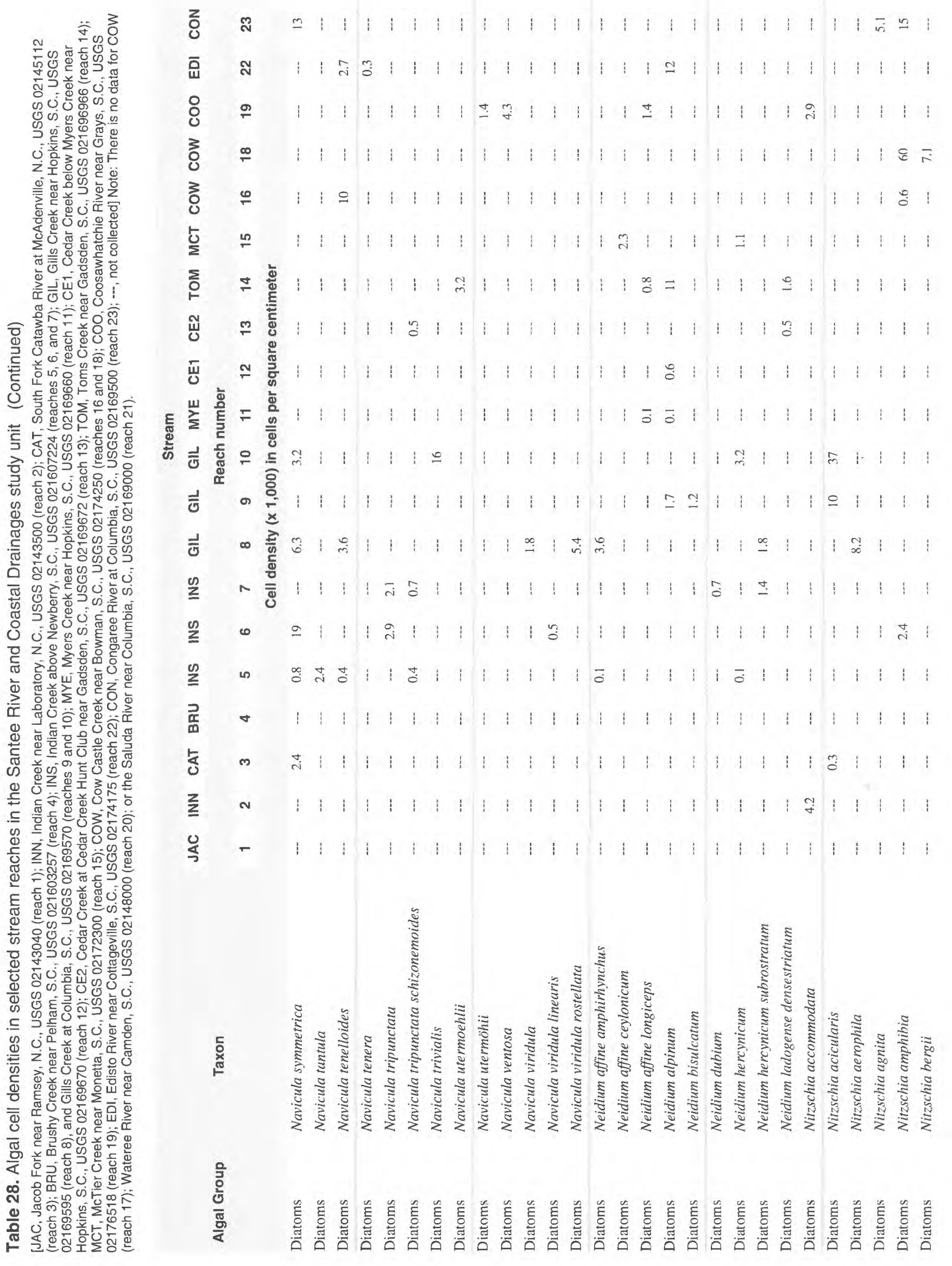


ำ 苛氙

舟航

定

i

代

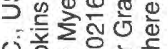

응

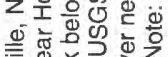

部娄

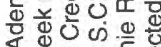

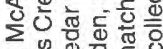

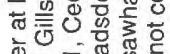

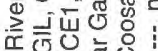

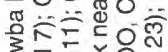

ఫิ

足 000

눈

过 辛

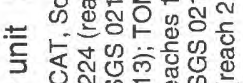

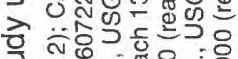

类

ש

苋

亲 品

娄

$\bar{\pi}$ on

एٓ

ठำ

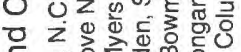

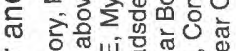

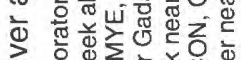

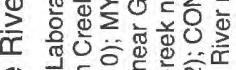

过

焉

ஸ

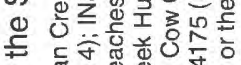

$\subseteq$ 需

\&

๘

秀

인

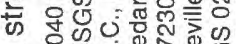

व

过

\& के हुण

क

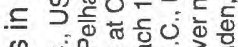

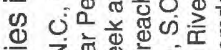

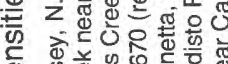

ठ

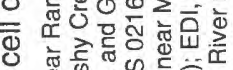

ब

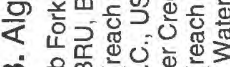

尔

N

응
ठ ๙

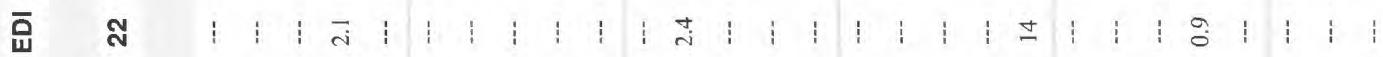

8 유

苂

¿

Łั

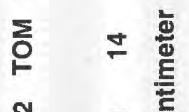

约

ธิบ ํํำ

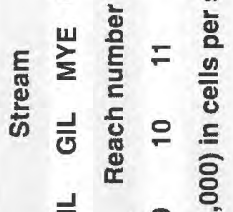

궁

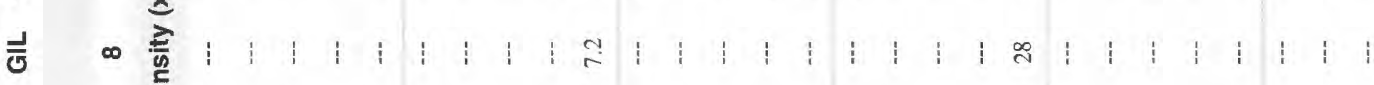

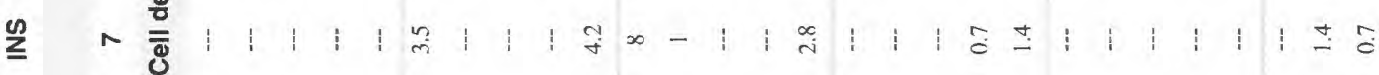

$\stackrel{0}{z} \quad 0$

$\underline{\Perp}$ ผ

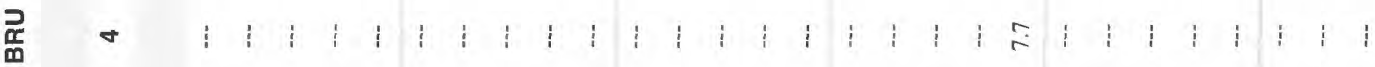

志 m :

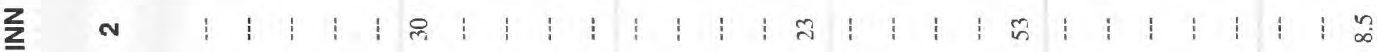

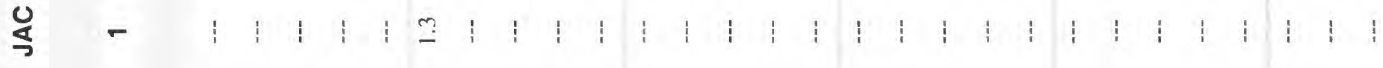

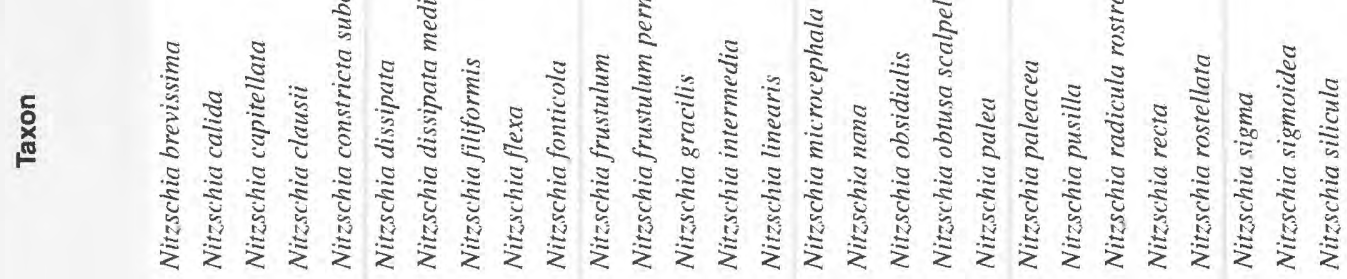




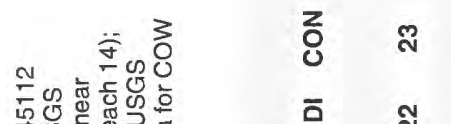

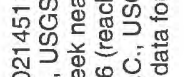

of

So

S)

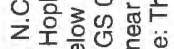

舫

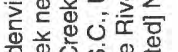

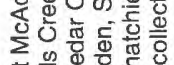

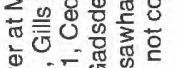

这议造造:

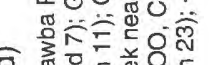

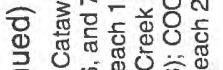

蛋

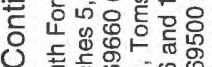

U 吾

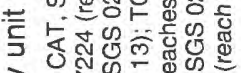

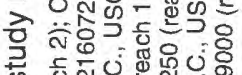

娄

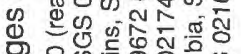

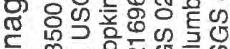

둔

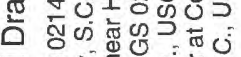

雨

ญิ

응

它

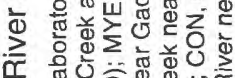

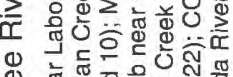

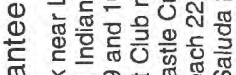

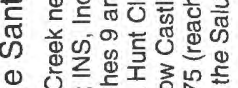

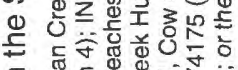

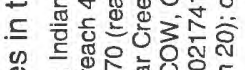

要

证

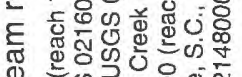

要

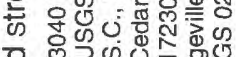

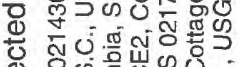

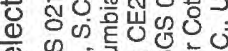

要

$\leq$

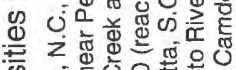

音

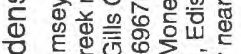

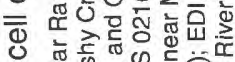

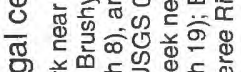

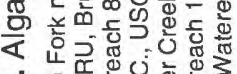

งิ

o

은

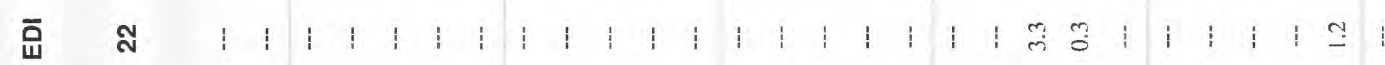

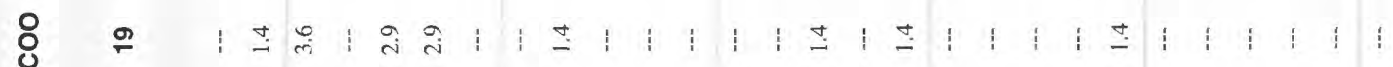

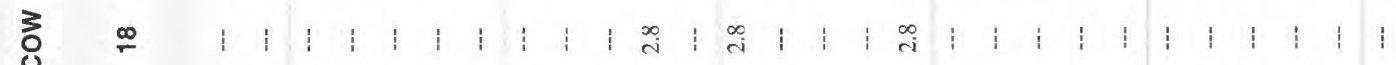

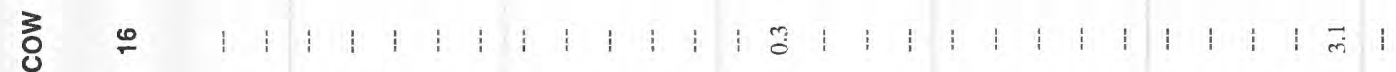

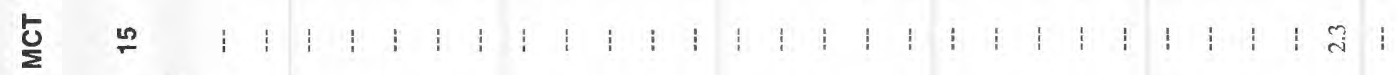

气

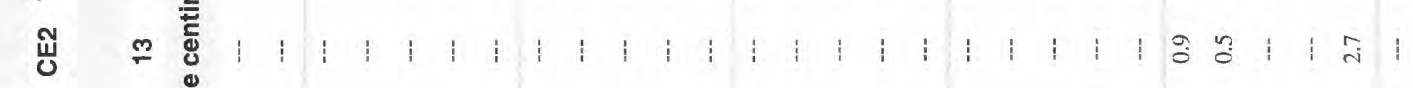

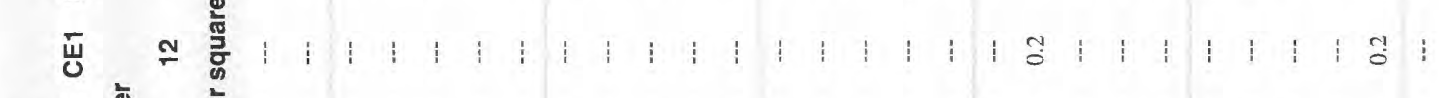

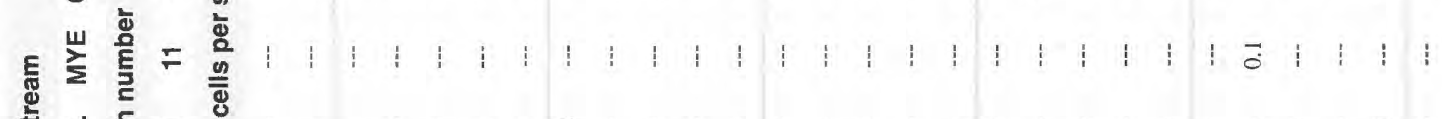

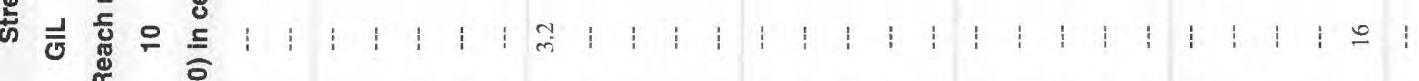

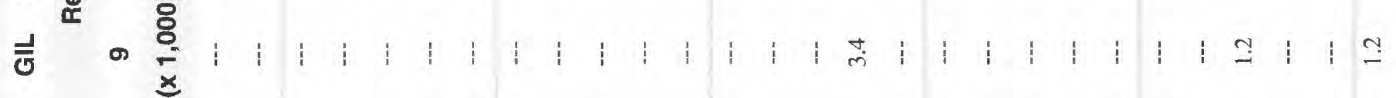

ప

$\stackrel{\infty}{\geqq}$ ค 焉:

$\stackrel{\infty}{\leftrightarrows} 0$ : 1 1 : 1 : : :

象

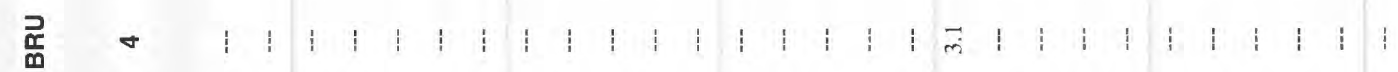

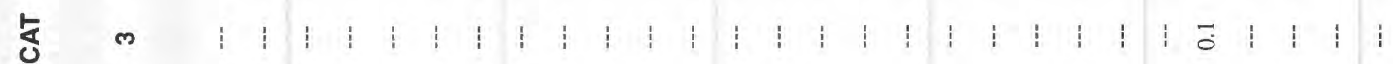

z $\quad$ 人

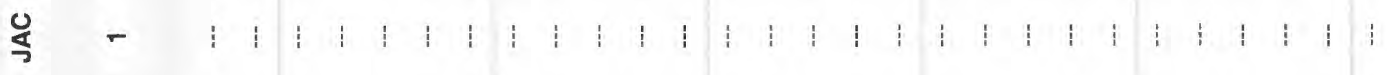

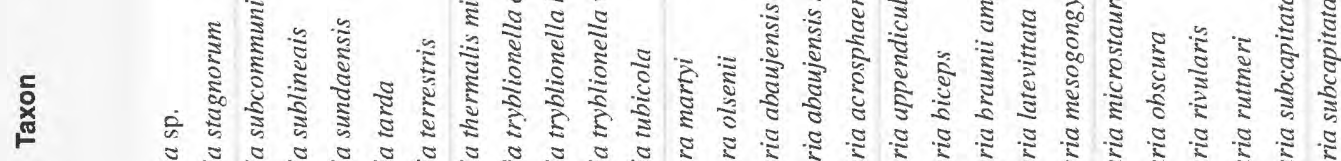

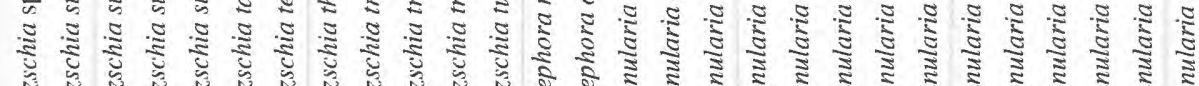

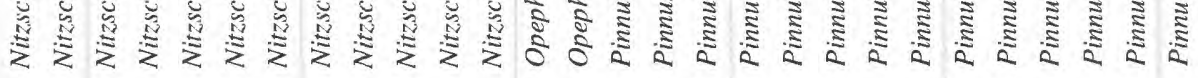

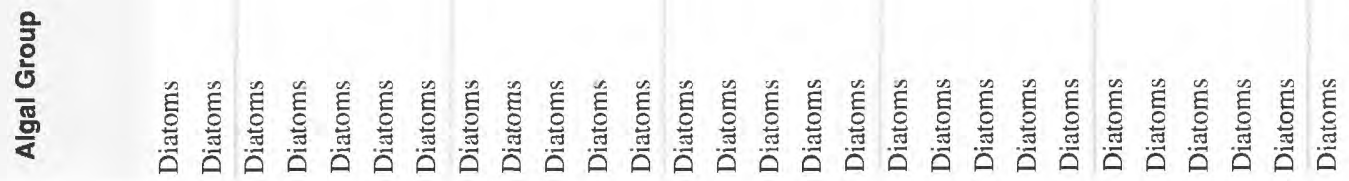


ㄲ.

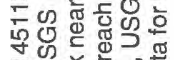

放

क人 ⿰⿺乚一兀)

जु

ᄀ

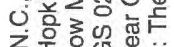

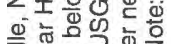

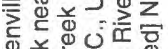

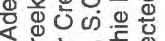

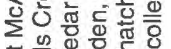

त

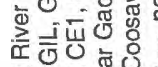

จ

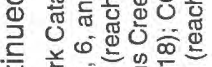

든

U 言

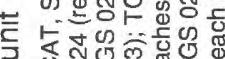

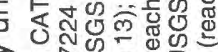

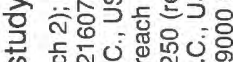

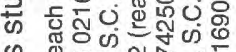

\&

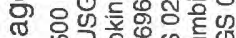

此 告

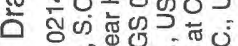

ब记

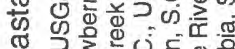
ठำ

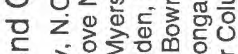

ฮ

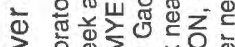

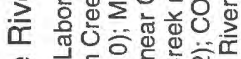

\&

空

( )

品

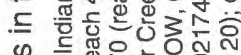

¿ ż

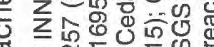

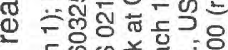

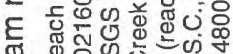

o

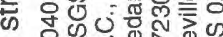

ס \%

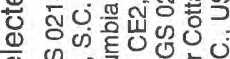

क O

.든

œ 00 \%

क

要

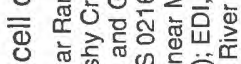

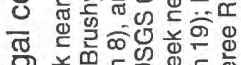

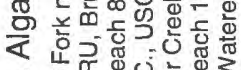

กิ

0 两

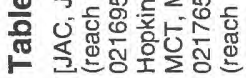

ㅇํㅇ ก

ํำ

융

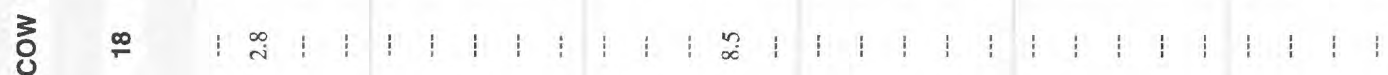

产

过

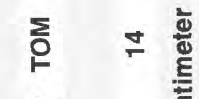

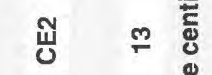

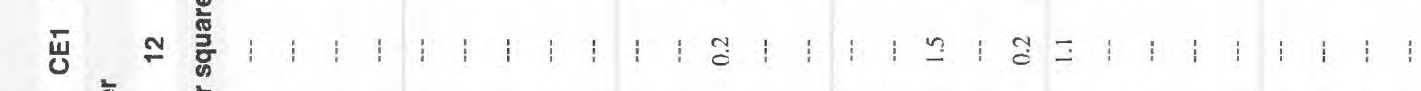

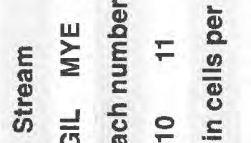

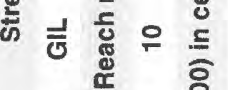

एँ

a 응

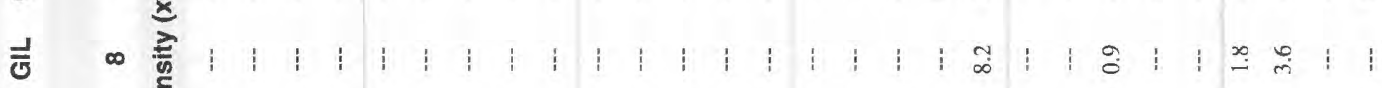

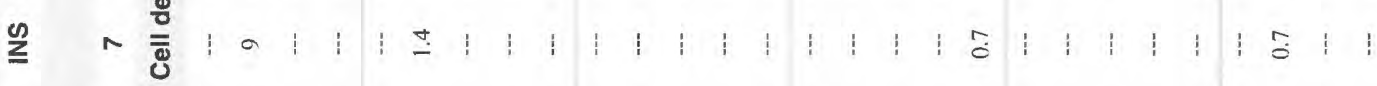

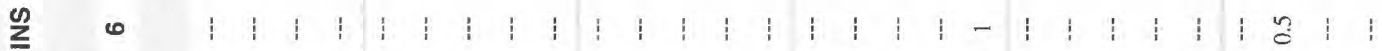

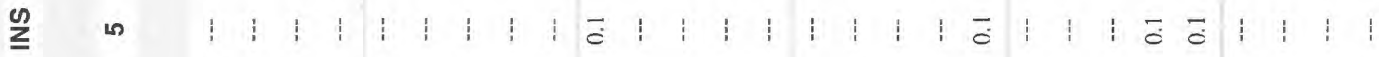

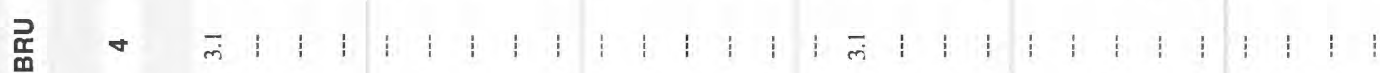

趑 m :

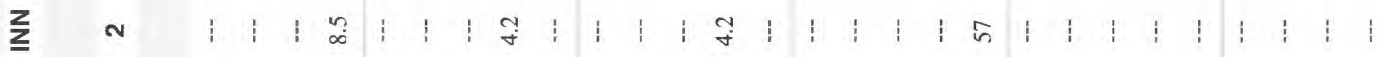

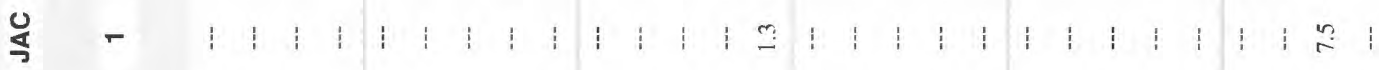

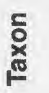

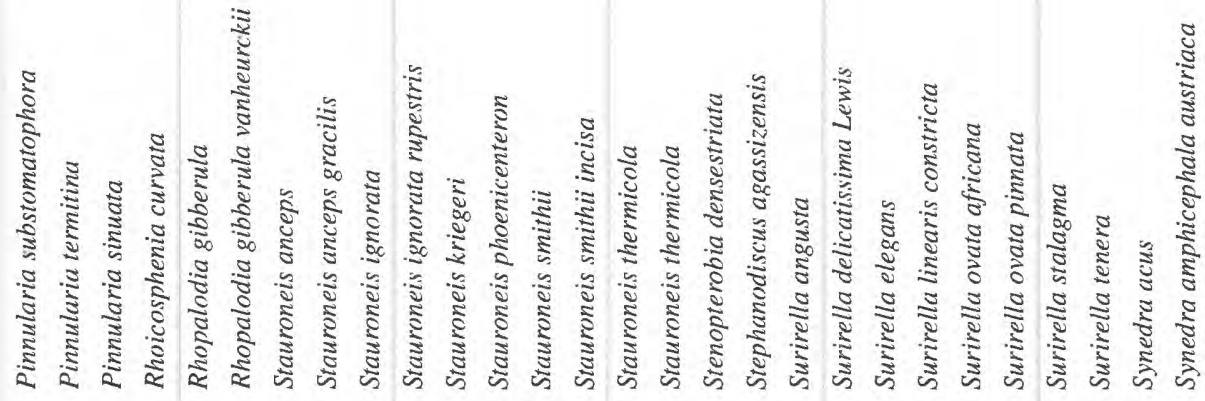

$\frac{\frac{0}{8}}{\frac{0}{0}}$

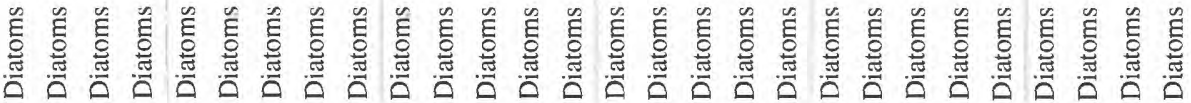




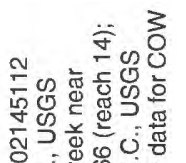

讨

जु

ᄀ

능간

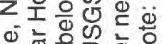

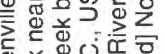

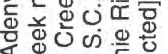

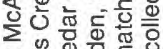

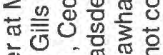

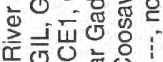

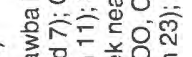

O

类 60

단

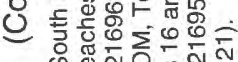

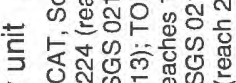

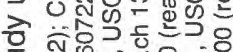

蛋

\&

\% 80 흥응

춘

市

ब

क्.

$\mathcal{O}$ 今

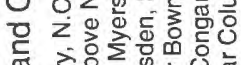

完

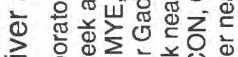

ॠ

\&

壳

क

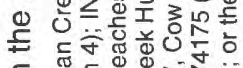

$\subseteq$ 呚

\&

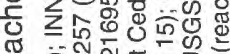

屯

E

는

क o

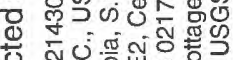

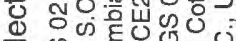

过

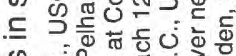

D

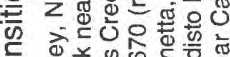

要

近

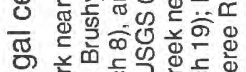

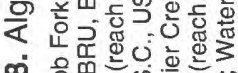

ô

告
วั ะ

บิ ส

응 으

ํํㅇ

응

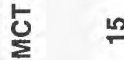

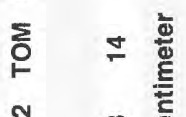

ษึ

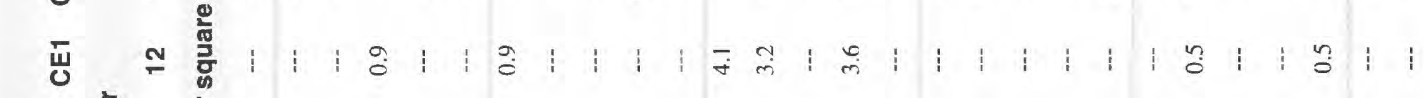

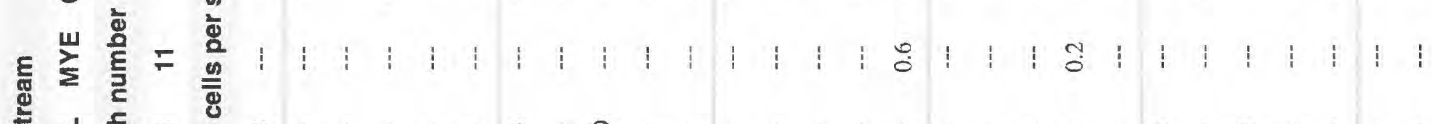

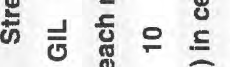

गे

$\frac{1}{0}$

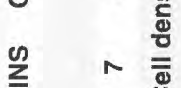

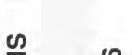

on is

귬

歺 m :

$\geqq$

迎

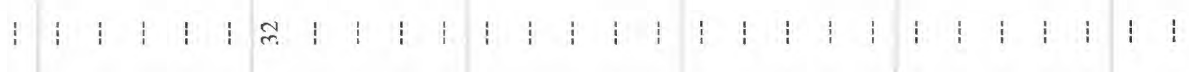

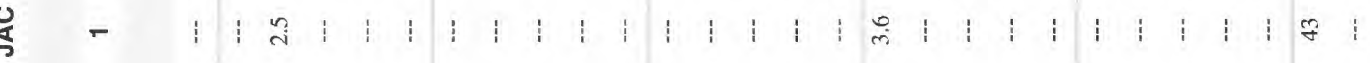

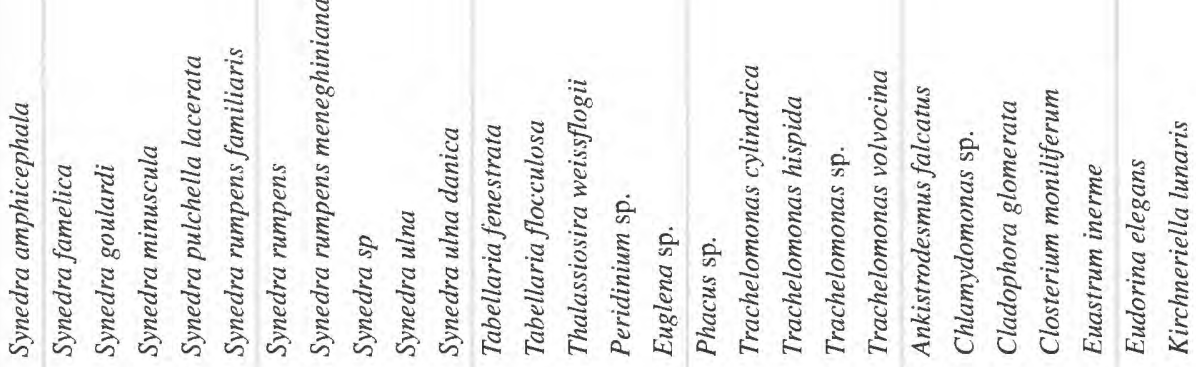

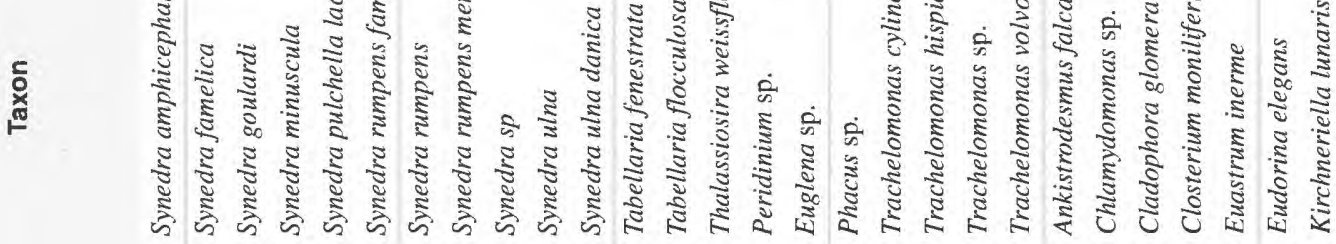

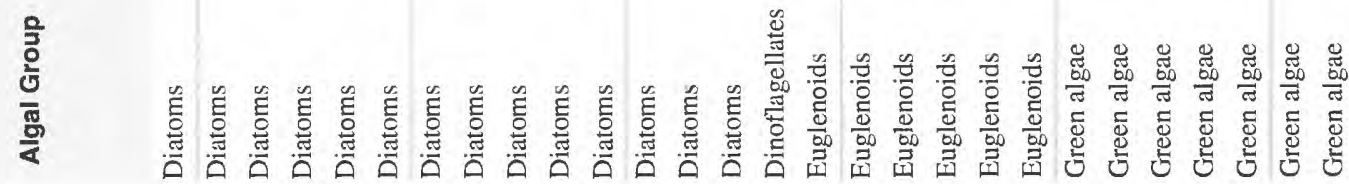


에

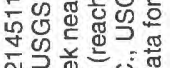

放

on 0 \%

जि

今.

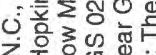

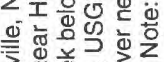

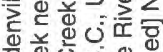

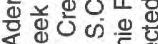

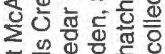

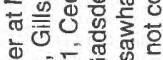

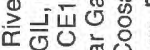

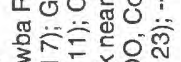

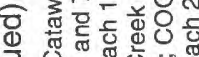

万人 0

단

O

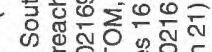

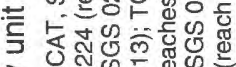

흐 숭응

फ 듕心

ש

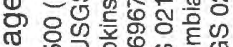

恋 㶽

西

ब

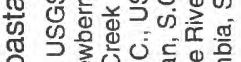

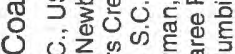

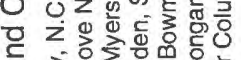

ส

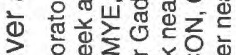

त

ه

突

离

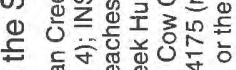

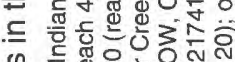

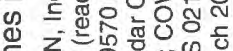

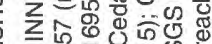

议

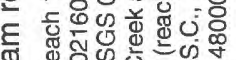

要

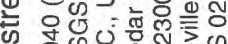

o \%

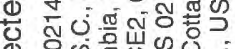

D

$\subseteq$ 范

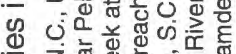

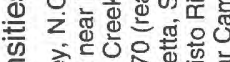

d

응

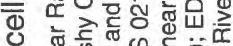

ब

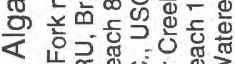

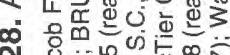

สิ

告
ชั

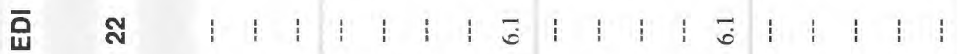

8ㅇ

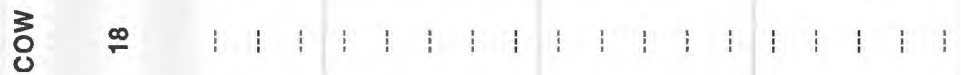

言

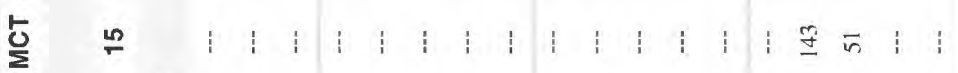

รัด

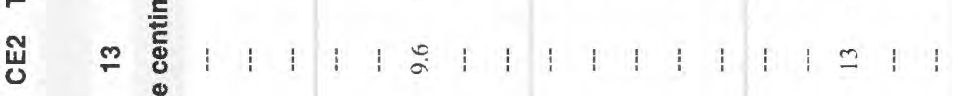

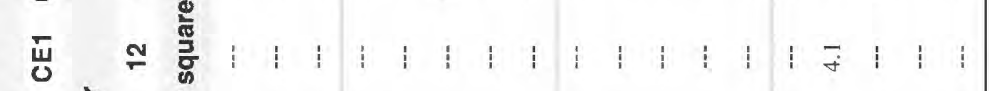

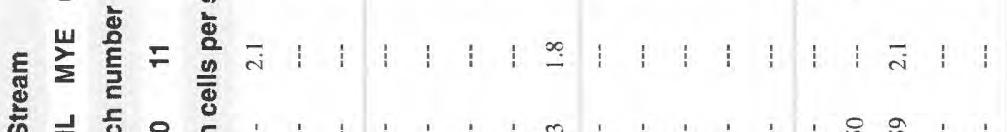

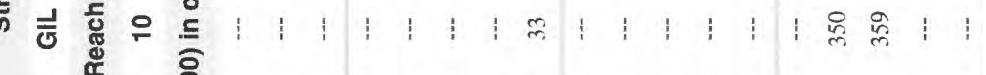

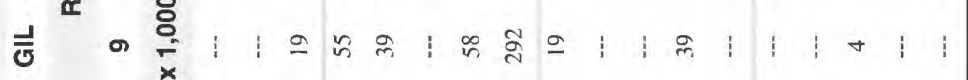

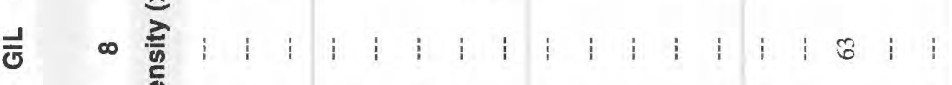

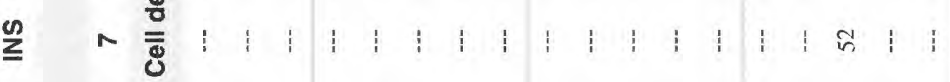

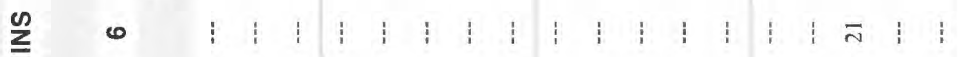

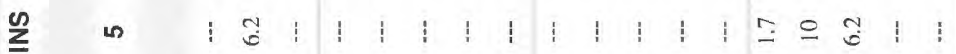

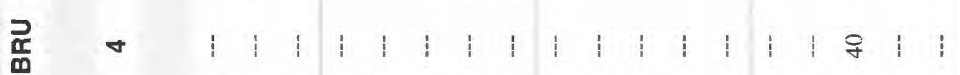

冬

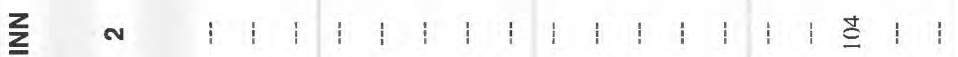

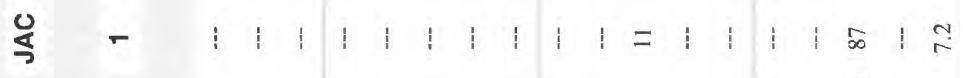

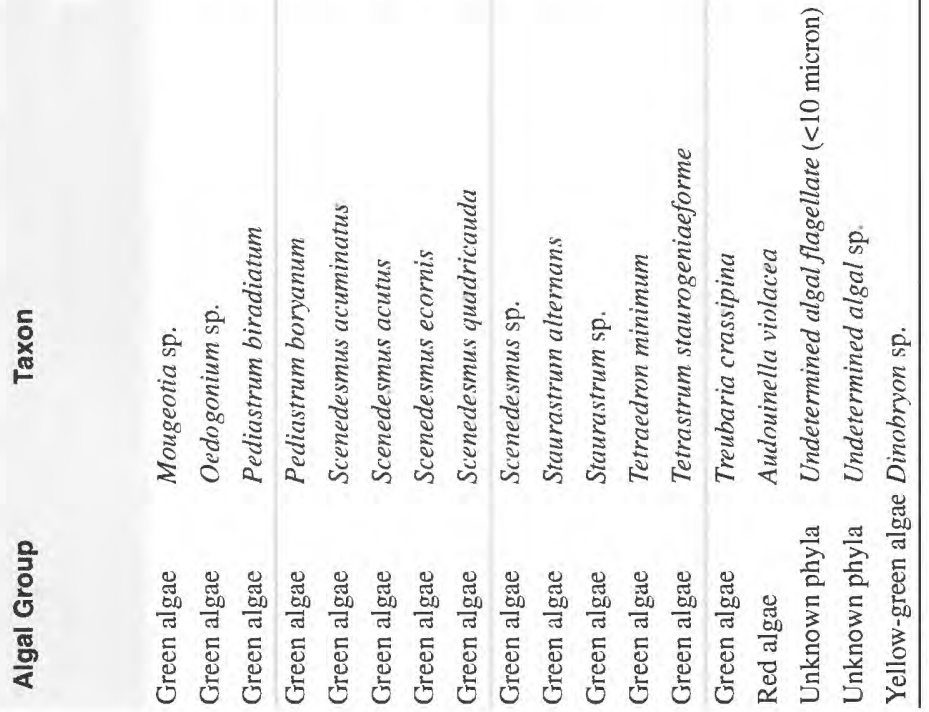




\section{Benthic Invertebrate Data}


Table 29. Benthic invertebrate density and diversity in the Santee River Basin and Coastal Drainages study unit

\begin{tabular}{|c|c|c|c|}
\hline Reach & Stream & $\begin{array}{c}\text { Organisms } \\
\text { per square } \\
\text { meter of } \\
\text { substrate }\end{array}$ & $\begin{array}{c}\text { Total number of } \\
\text { benthic } \\
\text { invertebrate } \\
\text { taxa }\end{array}$ \\
\hline 1 & Jacob Fork near Ramsey, N.C., USGS 02143040 & 1,363 & 107 \\
\hline 2 & Indian Creek near Laboratory, N.C., USGS 02143500 & 1,919 & 80 \\
\hline 3 & $\begin{array}{l}\text { South Fork Catawba River at McAdenville, N.C., USGS } \\
02145112\end{array}$ & 922 & 51 \\
\hline 4 & Brushy Creek near Pelham, S.C., USGS 021603257 & 1,252 & 67 \\
\hline 5 & Indian Creek above Newberry, S.C., USGS 021607224 & 1,106 & 82 \\
\hline 6 & Indian Creek above Newberry, S.C., USGS 021607224 & 834 & 72 \\
\hline 7 & Indian Creek above Newberry, S.C., USGS 021607224 & 2,123 & 67 \\
\hline 8 & Gills Creek near Hopkins, S.C., USGS 02169595 & 6,404 & 77 \\
\hline 9 & Gills Creek at Columbia, S.C., USGS 02149570 & 21,560 & 50 \\
\hline 10 & Gills Creek at Columbia, S.C., USGS 02149570 & 9,802 & 67 \\
\hline 11 & Myers Creek near Hopkins, S.C., USGS 02169660 & 11,327 & 84 \\
\hline 12 & $\begin{array}{l}\text { Cedar Creek below Myers Creek near Hopkins, S.C. USGS } \\
02169670\end{array}$ & 4.916 & 81 \\
\hline 13 & $\begin{array}{l}\text { Cedar Creek at Cedar Creek Hunt Club near Gadsden, S.C., } \\
\text { USGS } 02169672\end{array}$ & 12,311 & 90 \\
\hline 14 & Toms Creek near Gadsden, S.C., USGS 021696966 & 3,363 & 87 \\
\hline 15 & McTier Creek near Monetta, S.C., USGS 02172300 & 1,498 & 73 \\
\hline 16 & Cow Castle Creek near Bowman, S.C., USGS 02174250 & 1,422 & 68 \\
\hline 17 & Cow Castle Creek near Bowman, S.C., USGS 02174250 & 784 & 58 \\
\hline 18 & Cow Castle Creek near Bowman, S.C., USGS 02174250 & 4,946 & 58 \\
\hline 19 & Coosawhatchie River near Grays, S.C., USGS 02176518 & 12,104 & 85 \\
\hline 20 & Wateree River near Camden, S.C., USGS 02148000 & 4,255 & 23 \\
\hline 21 & Saluda River near Columbia, S.C., USGS 02169000 & 3,745 & 17 \\
\hline 22 & Edisto River near Cottageville, S.C., USGS 02174175 & 2,096 & 69 \\
\hline 23 & Congaree River at Columbia, S.C., USGS 02169500 & 2,969 & 89 \\
\hline
\end{tabular}




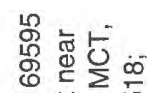

웡 훙 गुण S. ن

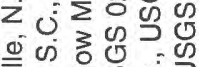

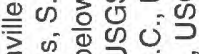

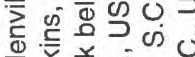
这尊安 오잉

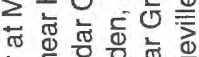

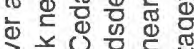
年

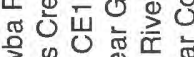

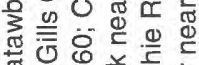

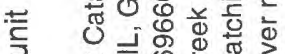
든 단 0

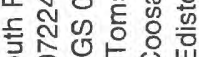
ㅇㅇㅇ 0 की 어요 ón

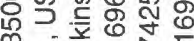

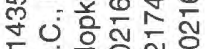
तิ in

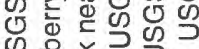
क i

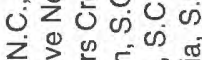

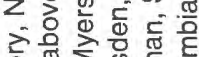
的

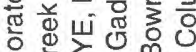

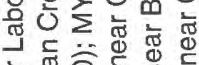
는

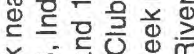

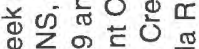

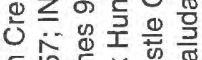

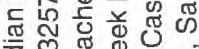

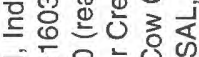

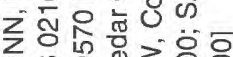

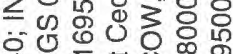
就的 0 尊

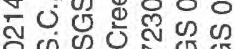
o o

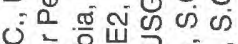
之 क 구의

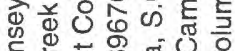

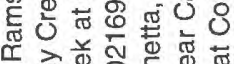

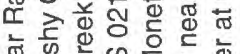

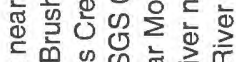

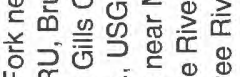
客

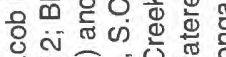
谓

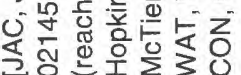

엉

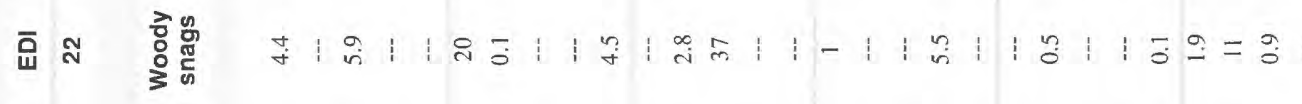

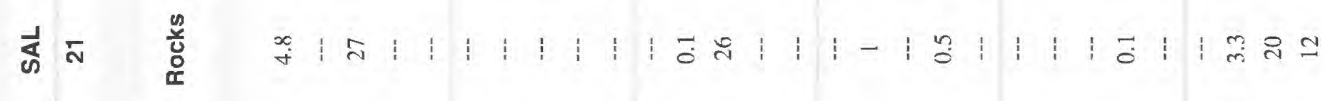

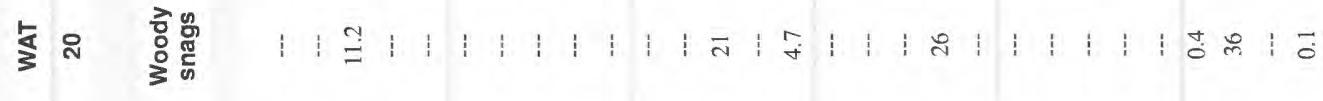

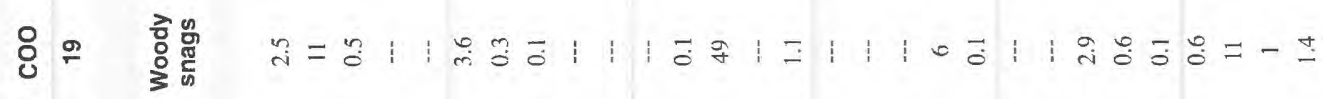

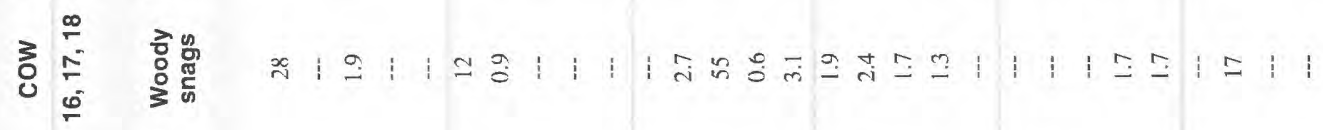

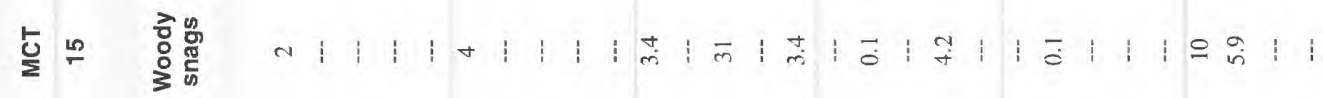

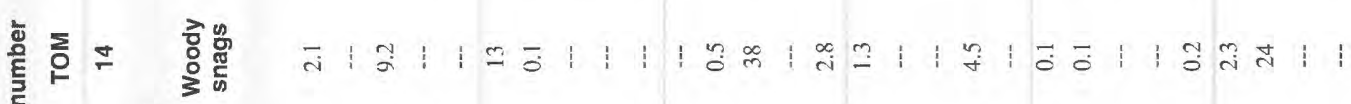

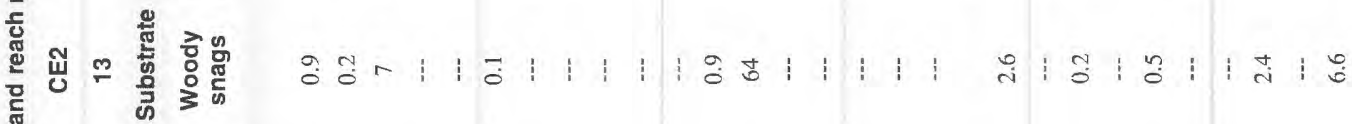

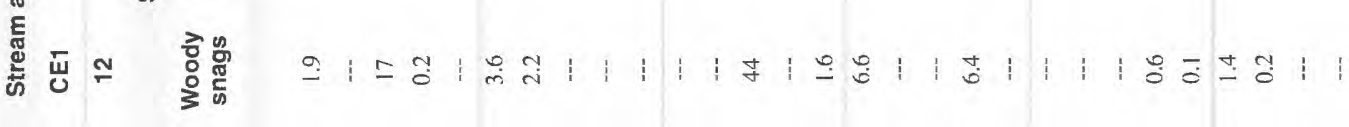

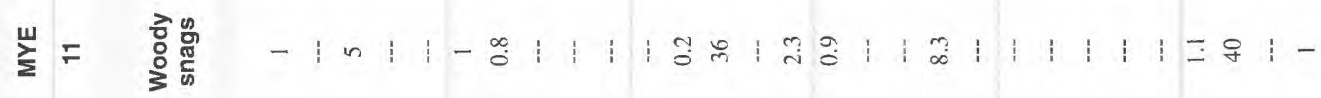

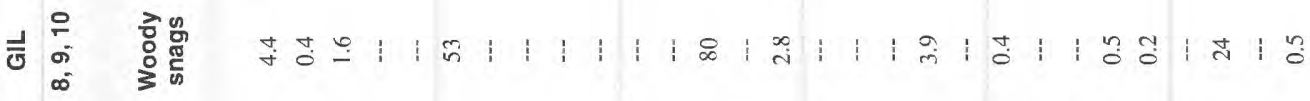

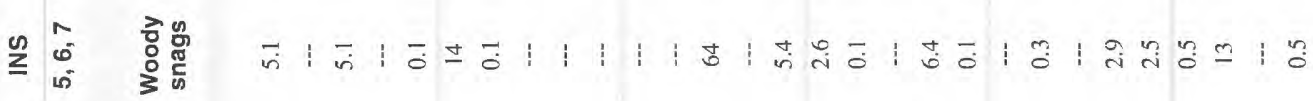

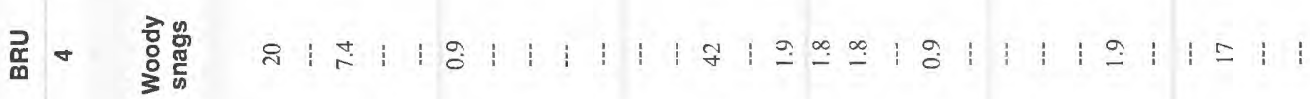

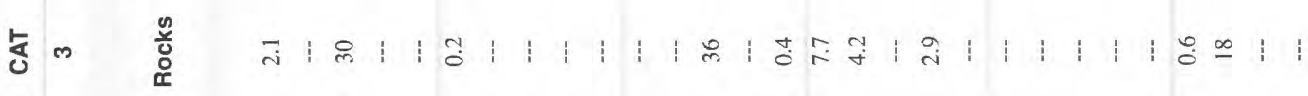

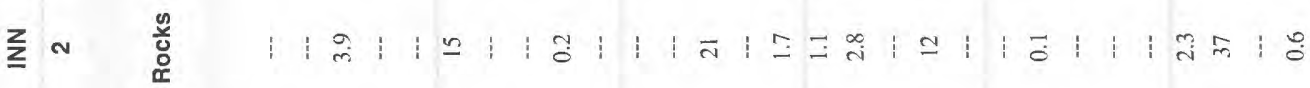

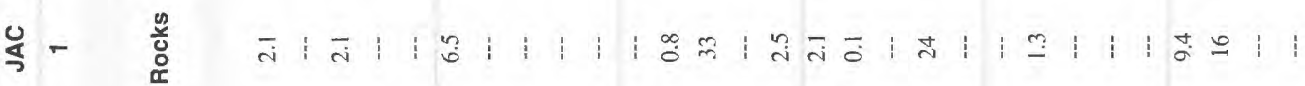

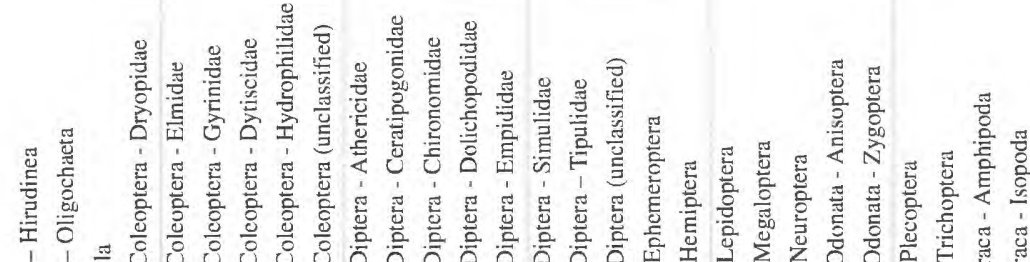

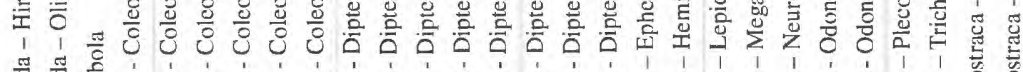

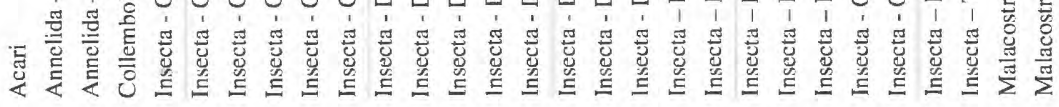


号㐫占

$0 \div$ 용

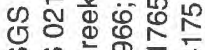

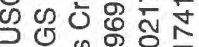

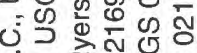

Z

ब

क

क्षे

응

$\sum$ 0

๙

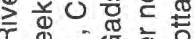

元

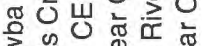

密灾

节 $00.0 \%$

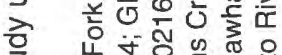

눙 है :

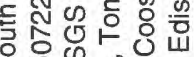

क ⿻ ㇒.

过

ठำ

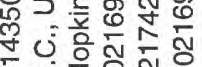

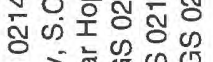

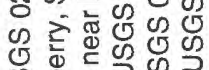

Oू

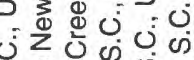

$z$ क ज

>े

政语

닌잉

త

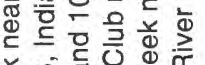

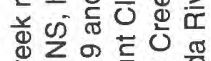

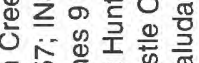

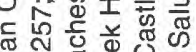

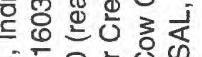

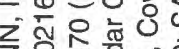

ஸ然家宫

\% 证

年

- 0 인잉

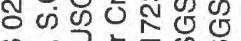

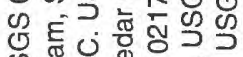

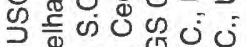

- ه

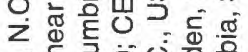

ब这的它

舟

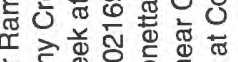

区

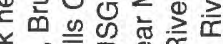

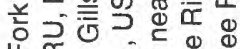

啠豞说

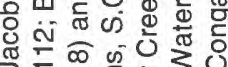

- 0230

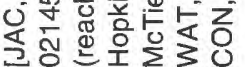

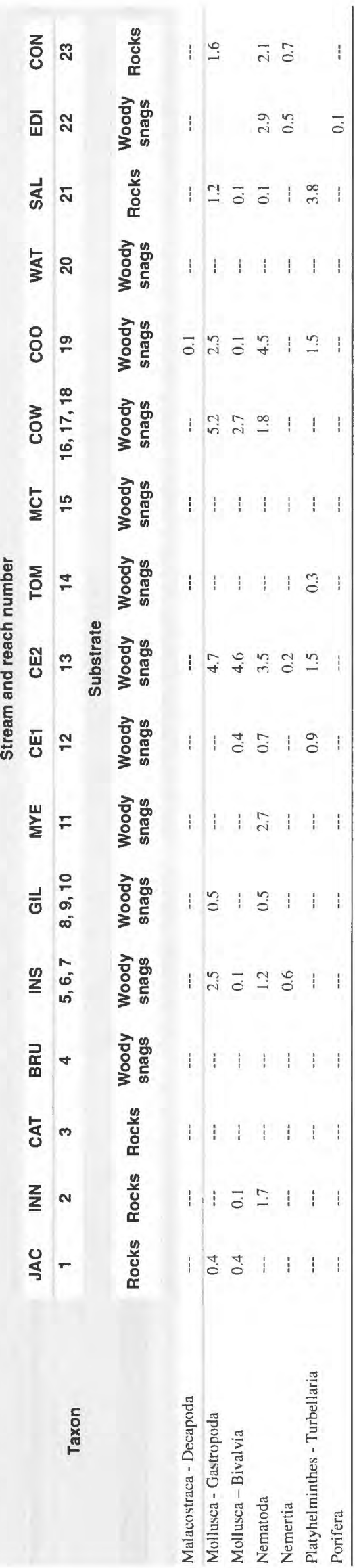


Fish Data 


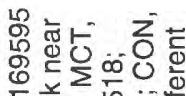
๘

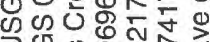
3. iิ

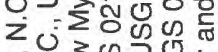
ब以 政

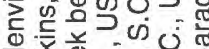

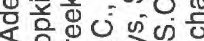
soris

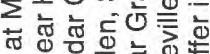

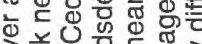

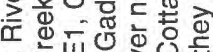

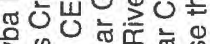

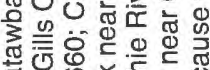

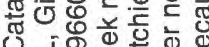

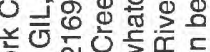

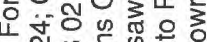
政此 列 की

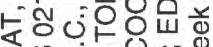
o fu w in 0 . 5 等 릉 on 政

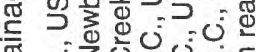

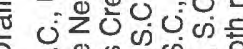
$\left.z 之 \frac{5}{5}\right)^{5}$ 㟧

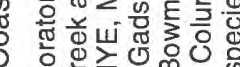

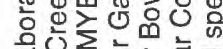

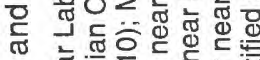
元

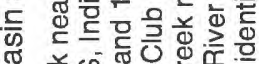
离

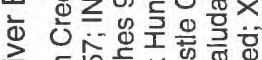

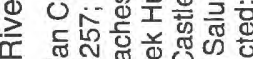
这 든 战 三人o

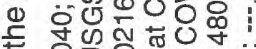

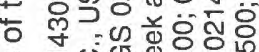

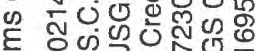
跣 कo

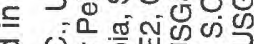

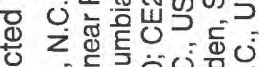

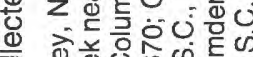

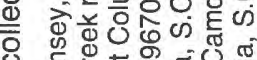

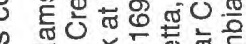
क्ष

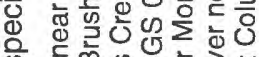
कo

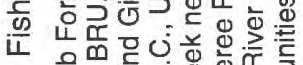

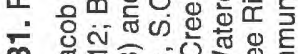
๑ 䒕

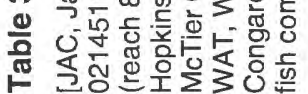

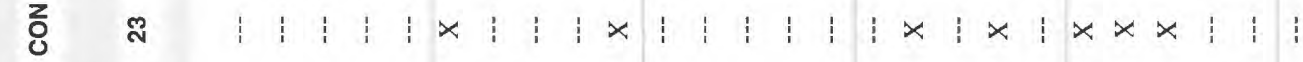
อิด

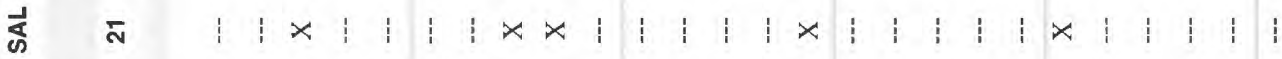
冬

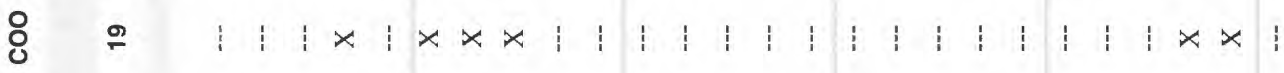
总

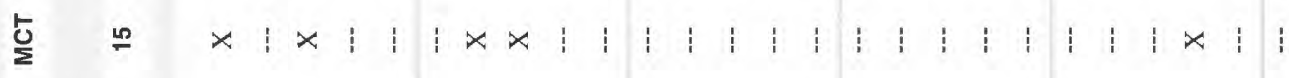
ฏ 离

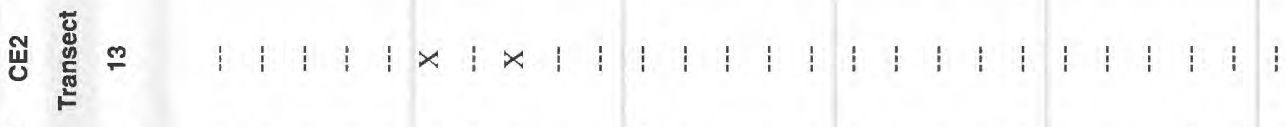

㟧 ำ

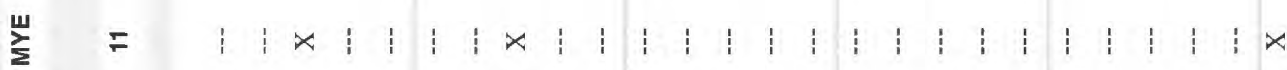

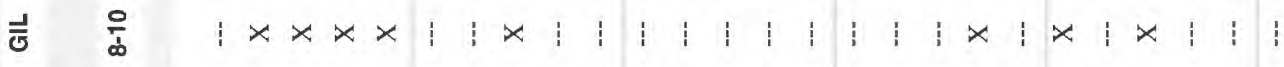

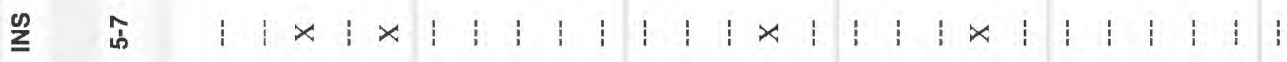

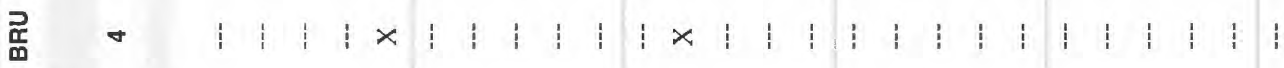
歺

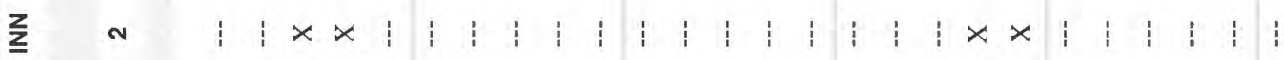

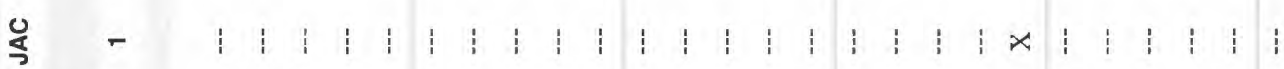

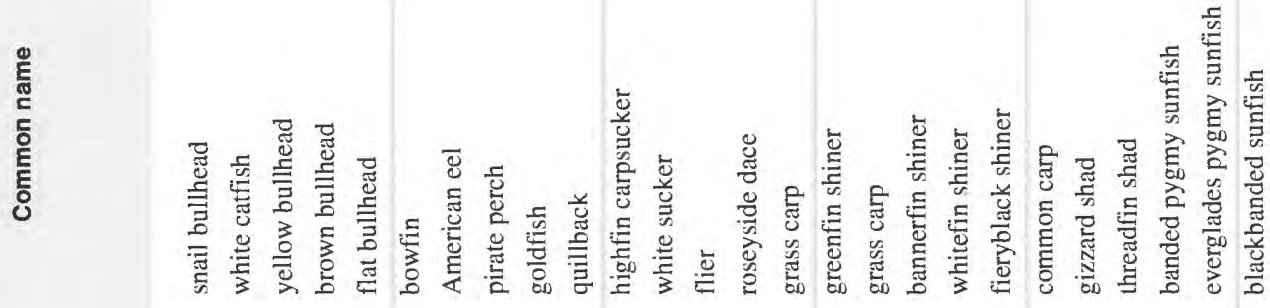

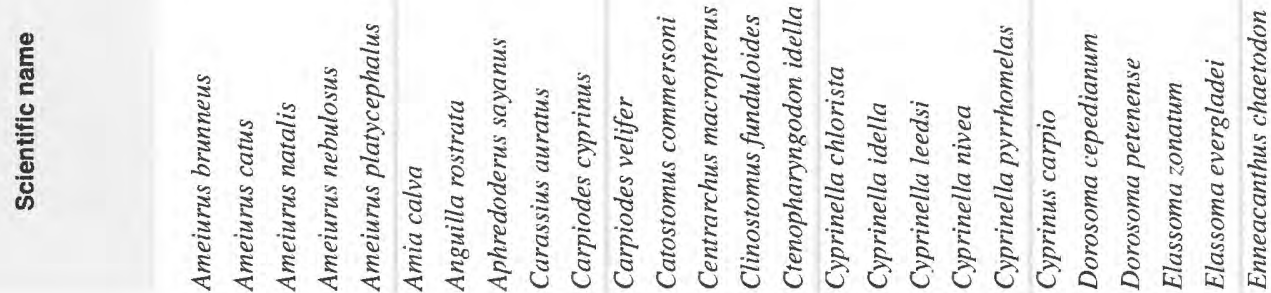




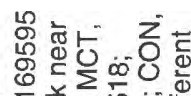

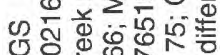

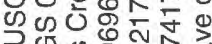
Jo ง ¿

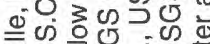

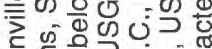
类

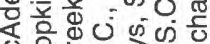

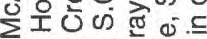

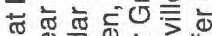

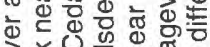

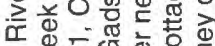

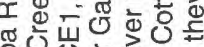

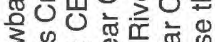

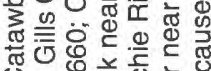
0 \% 잉

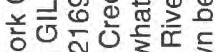
4 千 0 o

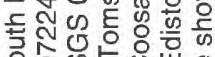
ㅇํㅇํㅇ인

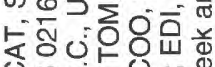
OSW 今

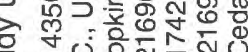
จั่

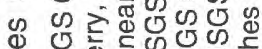

\%

ช $\supset 30$ 过 西 $z \$$

స्ञ

范

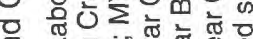

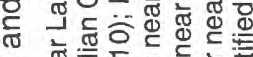

등

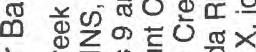

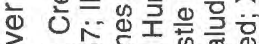

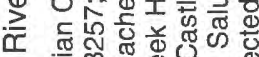

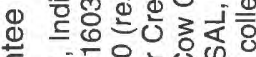

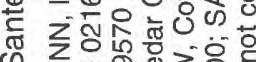

๓

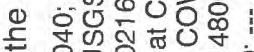

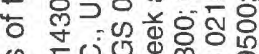

ह సั่

ॠ क E

के

$\subseteq \quad 0$.

¿ $z$ \&

Z

क

O

\& व

屯

के

年

iᄂ

的

0 工

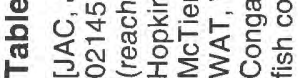

ర్ర

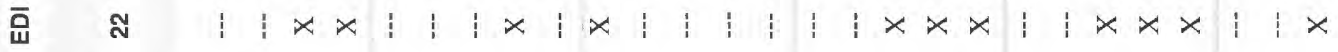

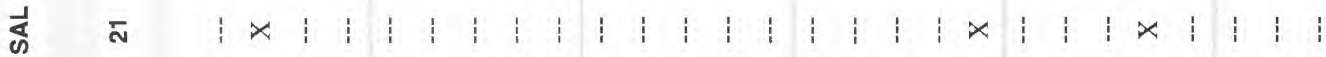

疋 인

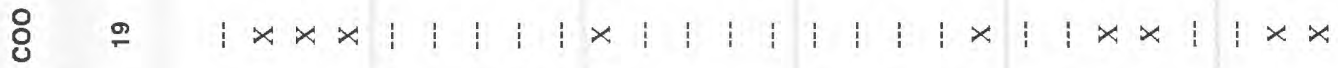
o Ł 产 \pm | $x \times x$ :

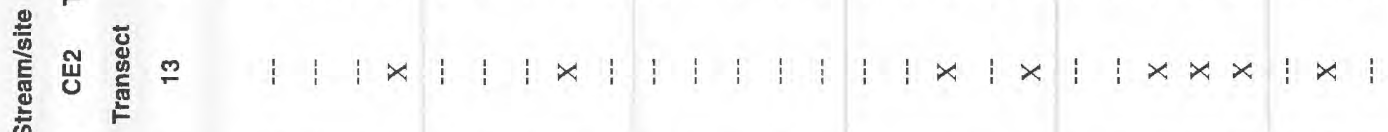
㟧 $\cong x:$ : 崖 = $=$ :

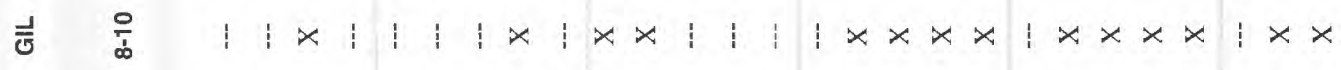
@ in $\mid x \times$ : 兴 $+\quad$ : 过 $m$ :

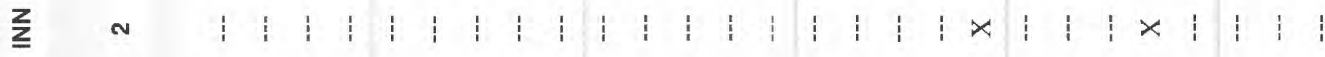

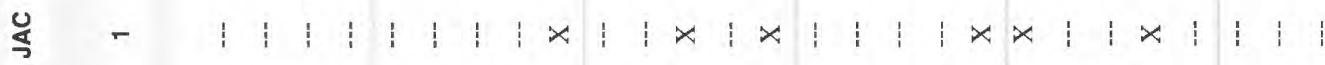

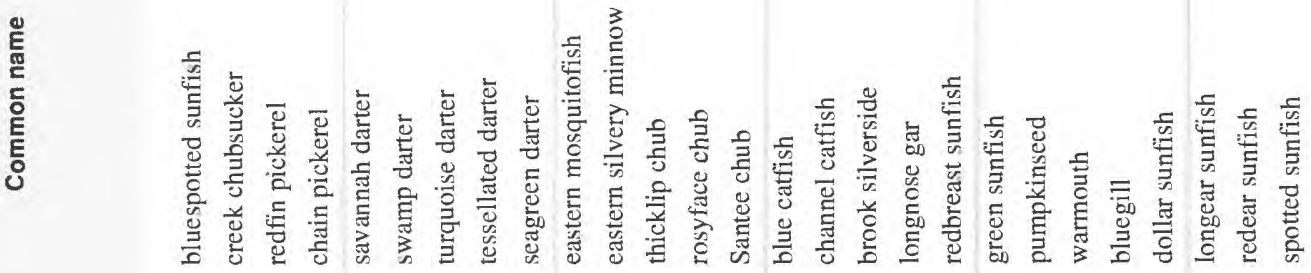

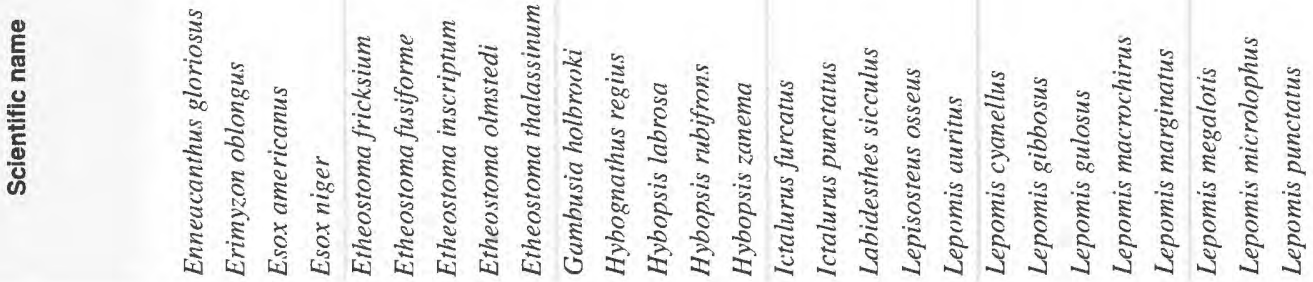




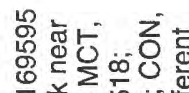

$\omega$ 宊

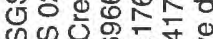

ग吅比市

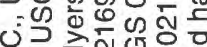

Z

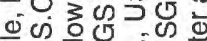

क

든

号守

它응

ส

๖ ฮ

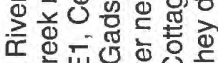

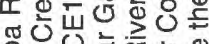

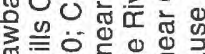

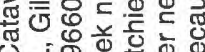

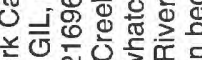

능

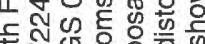

독

ஸ日

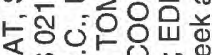

U

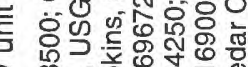

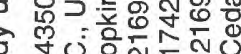
뜽

แ

O

过兄过

Z

ซ্ড

훈

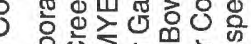

은

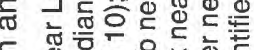

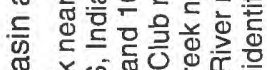

๓

๖

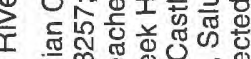

凹

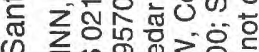

凹

ั.

凹

ह 0 以

Ф

の

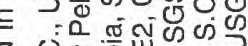

¿

$z$ \&

đ)

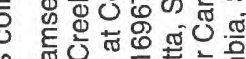

Ф ๙

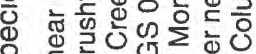

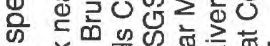

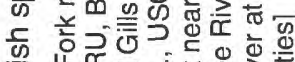

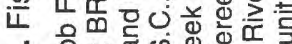

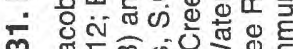

๓ 宁宁

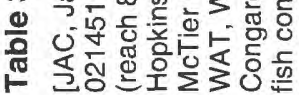

z

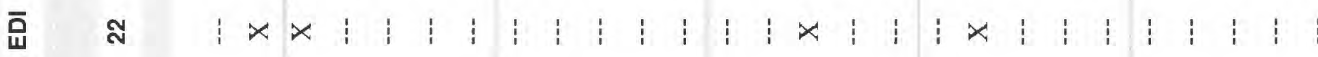

का

桌

윰

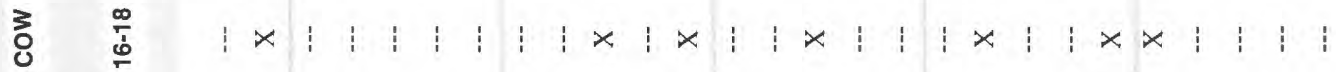

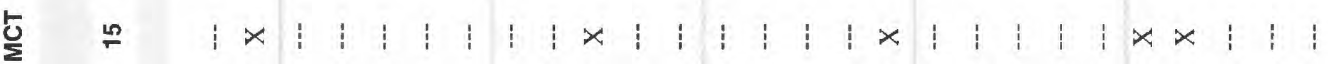

产

咅

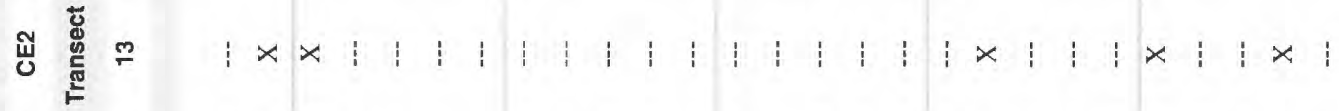

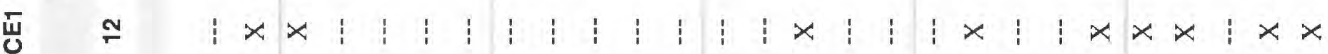

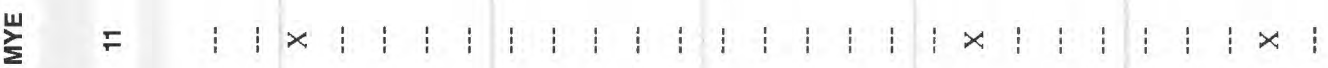

궁 $\frac{0}{0}$ :

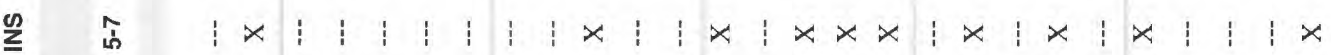

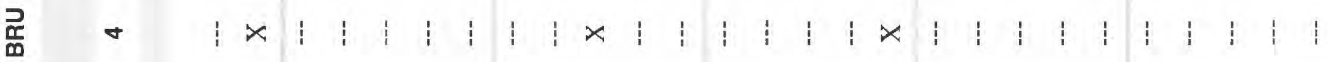

歺

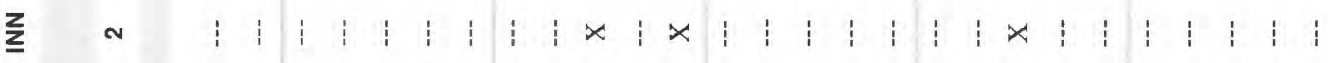

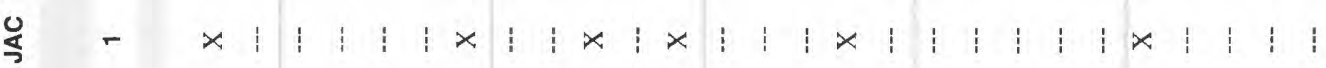

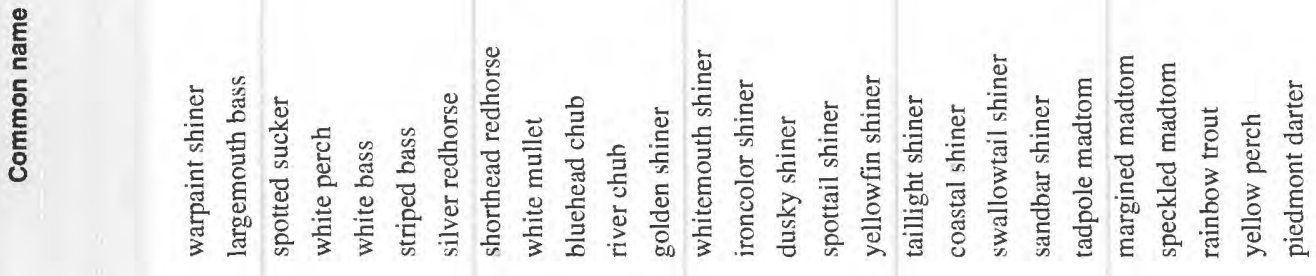

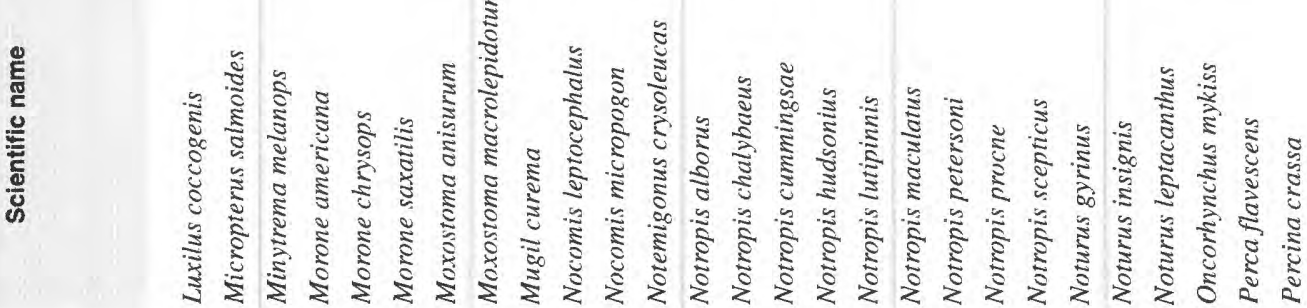




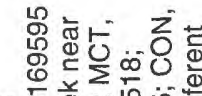

o  is

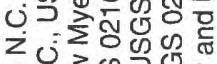
U उ क 00

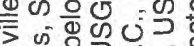

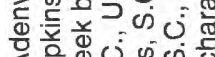

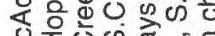

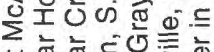

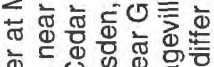

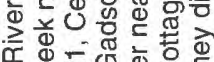

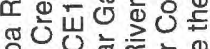
政 에 ज演

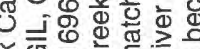
놓원원

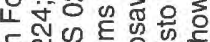

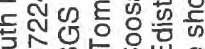

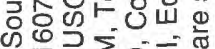

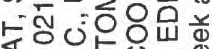
ơ

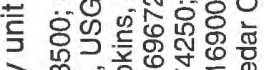

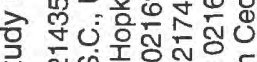
फ of की \% 员

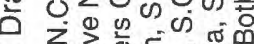

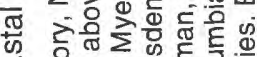

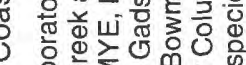
造之一巛口

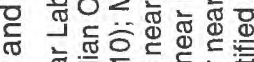
든

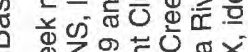

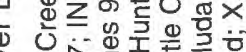
证

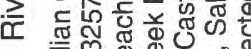

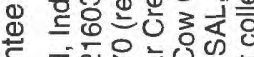

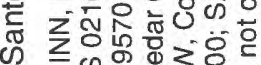
क \一

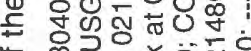
어에

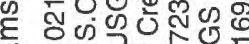

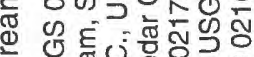
议 $\subseteq 3$ \% 匹

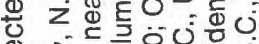
बें E क ष

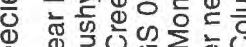
के

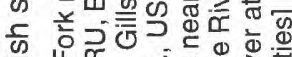
迹

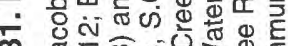
๓

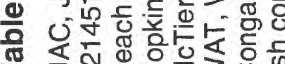

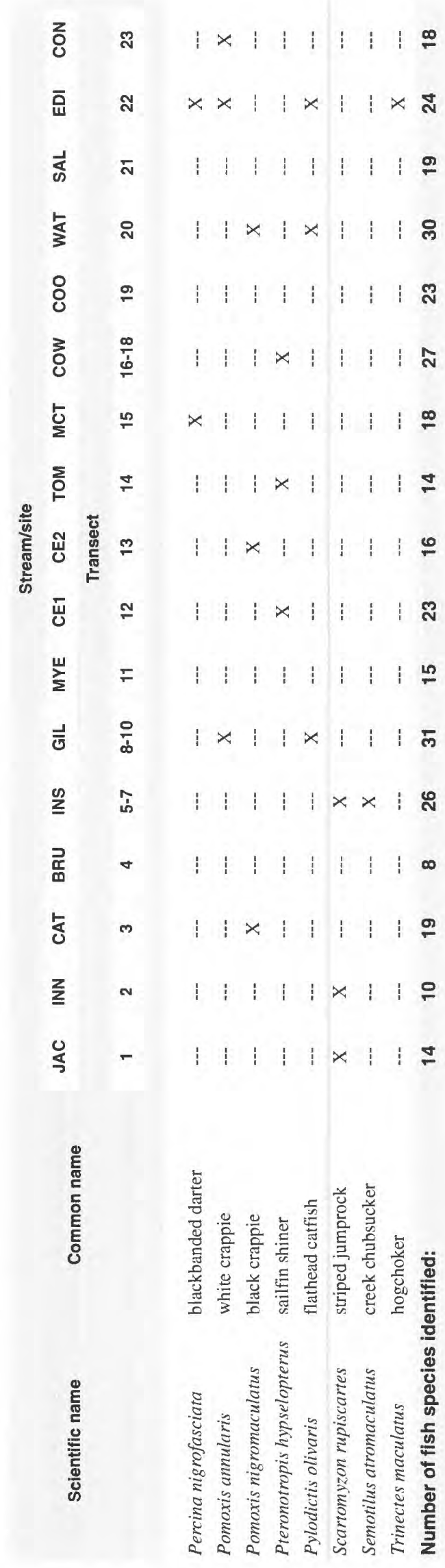

